\title{
DUST MASSES, PAH ABUNDANCES, AND STARLIGHT INTENSITIES IN THE SINGS GALAXY SAMPLE
}

\author{
B. T. Draine, ${ }^{1}$ D. A. Dale, ${ }^{2}$ G. Bendo, ${ }^{3}$ K. D. Gordon, ${ }^{4}$ J. D. T. Smith, ${ }^{4}$ L. Armus,${ }^{5}$ C. W. Engelbracht ${ }^{4}$ \\ G. Helou, ${ }^{6}$ R. C. Kennicutt, JR., ${ }^{4,7}$ A. Li, ${ }^{8}$ H. Roussel, ${ }^{9}$ F. Walter, ${ }^{9}$ D. Calzetti, ${ }^{10}$ J. Moustakas, ${ }^{4,11}$ \\ E. J. Murphy, ${ }^{12}$ G. H. Rieke, ${ }^{4}$ C. Bot, ${ }^{6}$ D. J. Hollenbach, ${ }^{13}$ K. Sheth, ${ }^{6}$ and H. I. Teplitz ${ }^{5}$ \\ Received 2007 January 16; accepted 2007 March 5
}

\begin{abstract}
Physical dust models are presented for 65 galaxies in SINGS that are strongly detected in the four IRAC bands and three MIPS bands. For each galaxy we estimate (1) the total dust mass, (2) the fraction of the dust mass contributed by PAHs, and (3) the intensity of the starlight heating the dust grains. We find that spiral galaxies have dust properties resembling the dust in the local region of the Milky Way, with similar dust-to-gas ratio and similar PAH abundance. The observed SEDs, including galaxies with SCUBA photometry, can be reproduced by dust models that do not require "cold" ( $T \lesssim 10 \mathrm{~K})$ dust. The dust-to-gas ratio is observed to be dependent on metallicity. In the interstellar media of galaxies with $A_{\mathrm{O}} \equiv 12+\log _{10}(\mathrm{O} / \mathrm{H})>8.1$, grains contain a substantial fraction of interstellar $\mathrm{Mg}$, Si, and Fe. Galaxies with $A_{\mathrm{O}}<8.1$ and extended $\mathrm{H}_{\mathrm{I}}$ envelopes in some cases appear to have global dust-to-gas ratios that are low for their measured oxygen abundance, but the dust-to-gas ratio in the regions where infrared emission is detected generally appears to be consistent with a substantial fraction of interstellar $\mathrm{Mg}, \mathrm{Si}$, and Fe being contained in dust. The PAH index $q_{\mathrm{PAH}}$, the fraction of the dust mass in the form of PAHs, correlates with metallicity. The nine galaxies in our sample with $A_{\mathrm{O}}<8.1$ have a median $q_{\mathrm{PAH}}=1.0 \%$, whereas galaxies with $A_{\mathrm{O}}>8.1$ have a median $q_{\mathrm{PAH}}=$ $3.55 \%$. The derived dust masses favor a value $X_{\mathrm{CO}} \approx 4 \times 10^{20} \mathrm{~cm}^{-2}\left(\mathrm{~K} \mathrm{~km} \mathrm{~s}^{-1}\right)^{-1}$ for the CO-to- $\mathrm{H}_{2}$ conversion factor. Except for some starbursting systems (Mrk 33, Tol 89, NGC 3049), dust in the diffuse ISM dominates the IR power.

Subject headings: dust, extinction — galaxies: abundances — galaxies: general — galaxies: ISM — infrared: galaxies - ISM: general

Online material: color figures, extended figure
\end{abstract}

\section{INTRODUCTION}

Interstellar dust affects the appearance of galaxies, by attenuating short-wavelength radiation from stars and ionized gas and contributing infrared (IR), far-infrared (FIR), submillimeter, millimeter, and microwave emission (for a recent review see Draine 2003). Dust also is an important agent in the fluid dynamics, chemistry, heating, cooling, and even ionization balance in some interstellar regions, with a major role in the process of star formation. Despite the importance of dust, determination of

\footnotetext{
1 Princeton University Observatory, Princeton, NJ 08544-1001; draine@astro .princeton.edu.

2 Department of Physics and Astronomy, University of Wyoming, Laramie, WY 82071; ddale@uwyo.edu.

3 Astrophysics Group, Imperial College, Blackett Laboratory, London SW7 2AZ, UK; g.bendo@imperial.ac.uk.

4 Steward Observatory, University of Arizona, Tucson, AZ 85721; kgordon@ as.arizona.edu, cengelbracht@as.arizona.edu,jdsmith@as.arizona.edu.

5 Spitzer Science Center, California Institute of Technology, Pasadena, CA 91125; lee@ipac.caltech.edu, hit@ipac.caltech.edu.

6 California Institute of Technology, Pasadena, CA 91125; gxh@ipac.caltech .edu,bot@caltech.edu.

7 Institute of Astronomy, University of Cambridge, Cambridge CB3 0HA, UK; robk@ast.cam.ac.uk.

8 Department of Physics and Astronomy, University of Missouri, Columbia, MO 65211; lia@missouri.edu.

9 Max-Planck-Institut für Astronomie, D-69117 Heidelberg, Germany; roussel@ mpia-hd.mpg.de, walter@mpia.de.

10 Space Telescope Science Institute, Baltimore, MD 21218; calzetti@stsci.edu.

11 Department of Physics, New York University, New York, NY 10003; john .moustakas@nyu.edu.

12 Department of Astronomy, Yale University, New Haven, CT 06520-8101; murphy@astro.yale.edu.

13 NASA/Ames Research Center, Moffett Field, CA 94035; hollenba@ism .arc.nasa.gov.
}

the physical properties of interstellar dust grains has been a challenging task: even the overall amount of dust in other galaxies has often been very uncertain.

The SINGS galaxy sample (Kennicutt et al. 2003) comprises 75 galaxies with a wide range of morphological types, all at distances allowing the galaxies to be resolved even at $160 \mu \mathrm{m}$. Observations of this sample with all instruments on the Spitzer Space Telescope (Werner et al. 2004) are providing a new perspective on the dust abundances and dust properties in a diverse set of galaxies.

For 67 of the SINGS galaxies we have positive detections in the four bands of the Infrared Array Camera (IRAC; Fazio et al. 2004) and the three bands of the Multiband Imaging Photometer for Spitzer (MIPS; Rieke et al. 2004), spanning wavelengths from 3.6 to $160 \mu \mathrm{m}$, the wavelength range within which dust radiates most of its power, including the polycyclic aromatic hydrocarbon $(\mathrm{PAH})$ emission features that are prominent in many galaxies; 65 of these galaxies are used in the present study. For 11 galaxies we have global fluxes measured with the IRS $16 \mu \mathrm{m}$ peak-up filter. For 17 of the 65 galaxies, global $850 \mu \mathrm{m}$ flux measurements are also available from the SCUBA camera on the JCMT.

The IR emission from dust depends not only on the amount of dust present but also on the rate at which it is heated by starlight. We develop a technique for estimation of dust masses and starlight intensities in galaxies and apply it to estimate the total dust mass $M_{\text {dust }}$ in each of the 65 galaxies in this study. For 61 galaxies we are also able to estimate a "PAH index" $q_{\mathrm{PAH}}$, defined here to be the mass fraction of the dust that is contributed by $\mathrm{PAH}$ particles containing $<10^{3} \mathrm{C}$ atoms. In addition, we obtain information on the starlight intensity distribution: the mean starlight intensity scale factor $\langle U\rangle$, and the fraction of the dust heating that takes place in regions of high starlight intensities, including photodissociation regions (PDRs). 
The paper is organized as follows. The sample selection and observational data are summarized in $\S 2$, the physical dust models that are used are described in $\S 3$, and the model-fitting procedure is given in $\S 4$. In order to validate the model-fitting procedure, we first limit attention to the subset of SINGS galaxies for which SCUBA data are available (we refer to these as the SINGS-SCUBA galaxies). In $\S 5$ we demonstrate that the dust modeling procedure works well for most of the SINGS-SCUBA galaxies, and we present dust masses, dust-to-gas mass ratios, PAH index values, and starlight intensity parameters obtained from these model fits. For the SINGS-SCUBA galaxies, our models do not require any contribution from the very cold $(<10 \mathrm{~K})$ dust that has been reported by Krugel et al. (1998) for the spiral galaxies NGC 6156 and NGC 6918, or the 5-9 K dust that Galliano et al. (2003, 2005) reported for low-metallicity dwarf galaxies, or the 4-6 K dust that Dumke et al. (2004) reported for the edge-on spiral NGC 4631. The global spectral energy distributions (SEDs) do not show evidence for the submillimeter excess seen in the faint outer regions of NGC 4631 (Bendo et al. 2006b).

Submillimeter measurements are valuable to help constrain the dust models but are unfortunately not available for most galaxies. In $\S 6$ we use the SINGS-SCUBA galaxies to develop a "restricted" fitting procedure that can be applied to galaxies for which submillimeter fluxes are unavailable. For the 17 galaxies with global $850 \mu \mathrm{m}$ fluxes from SCUBA, we show that the restricted fitting procedure, without employing SCUBA data, recovers dust masses and starlight intensities that are usually within a factor of 2 of the values obtained when the SCUBA data are used.

The restricted fitting technique is then applied to the remaining 48 galaxies to estimate dust masses and starlight intensities. Dust-to-gas mass ratios for the overall sample are presented in $\S 7.1$, where it is found that some of the SINGS galaxies have very low dust-to-gas ratios and that the dust-to-gas ratios are correlated with both gas-phase metallicity and galaxy type. The dustto-gas ratios depend on the assumed value of $X_{\mathrm{CO}}$, the ratio of $\mathrm{H}_{2}$ column density to $\mathrm{CO}$ line intensity; in $\S 9.4$ we argue that the distribution of derived dust-to-gas ratios supports $X_{\mathrm{CO}} \approx 4 \times$ $10^{20} \mathrm{~cm}^{-2}\left(\mathrm{~K} \mathrm{~km} \mathrm{~s}^{-1}\right)^{-1}$.

Values of the PAH index $q_{\mathrm{PAH}}$ are estimated for 61 galaxies in $\S$ 7.4. Many galaxies are found to have low values of $q_{\mathrm{PAH}}$, and $q_{\text {PAH }}$ is found to be strongly correlated with gas-phase metallicity. The starlight parameters for the 65 galaxy sample are presented and discussed in $\S 8$. The results are discussed in $\S 9$ and summarized in $\S 10$.

\section{DATA}

\subsection{IRAC and MIPS}

Spitzer was used to observe all 75 galaxies in the SINGS sample, following the observing strategy described in Kennicutt et al. (2003). The observations in the four IRAC bands $(3.6,4.5,5.7$, and $7.9 \mu \mathrm{m})$ and three MIPS bands $(24,71$, and $160 \mu \mathrm{m})$ have been reduced following the procedures described in Dale et al. (2005). The extended source aperture corrections found in Reach et al. (2005) were applied to the IRAC data. Global IRAC and MIPS photometry for these galaxies is taken from Dale et al. (2007), which included (usually modest) corrections for aperture size and for nonlinearity of the $71 \mu \mathrm{m}$ detectors.

Ten of the SINGS galaxies were excluded from the present study:

1. NGC 584 (E4) may be contaminated by a background source and also has low surface brightness in the MIPS bands compared to the foreground cirrus.
2. NGC 3034=M82 (I0, starburst): saturation of the bright core of this galaxy precludes global photometry.

3. NGC 1404 (E1): an off-center IR source contributes a significant fraction of the MIPS flux, but it is not known whether or not it is a foreground/background object.

4. NGC 4552 (E0) has low surface brightness compared to the foreground cirrus emission, and the MIPS $160 \mu \mathrm{m}$ photometry is very uncertain.

5. The dwarf galaxies M81dwA and Holmberg IX are not detected at $\lambda \geq 5.8 \mu \mathrm{m}$.

6. The dwarf galaxies DDO 154 and DDO 165 are only marginally $(<3 \sigma)$ detected at 71 and $160 \mu \mathrm{m}$, and M81dwB is a $<2 \sigma$ detection at $160 \mu \mathrm{m}$.

7. The dwarf starburst galaxy NGC 1377 shows strong silicate absorption at 9.8 and $18 \mu \mathrm{m}$ (Roussel et al. 2006). The objective of the present study is to model the IR spectra using a simple model that assumes the galaxy to be optically thin at $\lambda>3.5 \mu \mathrm{m}$; because NGC 1377 is clearly not optically thin, it has been removed from the sample.

The sample studied here therefore consists of 65 galaxies. MIPS $71 \mu \mathrm{m}$ photometry for NGC 7552 is not usable because of detector saturation, but NGC 7552 is retained in the sample because it is well observed at other wavelengths and with other instruments.

Most of the dust mass in galaxies is relatively cool, radiating primarily at wavelengths in the MIPS 71 and $160 \mu \mathrm{m}$ bands. Dust grains that are warmer, either because they are in regions with intense starlight or because they are very small grains immediately following absorption of a stellar photon, may radiate strongly in the MIPS $24 \mu \mathrm{m}$ band and shortward. The variety of dust conditions found in the SINGS sample is evident from the distribution of the 64 galaxies in MIPS color-color space, shown in Figure 1: $\left\langle\nu F_{\nu}\right\rangle_{71 \mu \mathrm{m}} /\left\langle\nu F_{\nu}\right\rangle_{160 \mu \mathrm{m}}$ varies by over a factor of 10 within our sample, and $\left\langle\nu F_{\nu}\right\rangle_{24 \mu \mathrm{m}} /\left\langle\nu F_{\nu}\right\rangle_{71 \mu \mathrm{m}}$ varies by a factor of 8 . These variations in MIPS colors presumably reflect galaxyto-galaxy differences in the intensity of the starlight heating the dust grains, although galaxy-to-galaxy differences in dust composition may also play a role. It is evident from the scatter in Figure 1 that the MIPS $24 \mu \mathrm{m} / 71 \mu \mathrm{m}$ color is not simply a function of the $71 \mu \mathrm{m} / 160 \mu \mathrm{m}$ color: the MIPS colors cannot be described by a one-parameter sequence of, e.g., "dust temperature." Realistic dust models will therefore need to vary at least two parameters to account for the range in observed MIPS colors.

The SINGS sample does not include any powerful active galactic nucleus (AGN), and in all SINGS galaxies the global flux in the IRAC $3.6 \mu \mathrm{m}$ band is starlight dominated. Neglecting extinction, we can estimate the "nonstellar" flux in longer wavelength bands by subtracting the estimated stellar contribution, assuming a $5000 \mathrm{~K}$ blackbody. For IRAC band 4, the IRS $16 \mu \mathrm{m}$ peak-up band (see $\S 2.2$ ), and the MIPS $24 \mu \mathrm{m}$ band, the nonstellar fluxes are

$$
\begin{aligned}
& \left\langle F_{\nu}^{\mathrm{ns}}\right\rangle_{7.9}=\left\langle F_{\nu}\right\rangle_{7.9}-0.260\left\langle F_{\nu}\right\rangle_{3.6}, \\
& \left\langle F_{\nu}^{\mathrm{ns}}\right\rangle_{16}=\left\langle F_{\nu}\right\rangle_{16}-0.0693\left\langle F_{\nu}\right\rangle_{3.6}, \\
& \left\langle F_{\nu}^{\mathrm{ns}}\right\rangle_{24}=\left\langle F_{\nu}\right\rangle_{24}-0.0326\left\langle F_{\nu}\right\rangle_{3.6} .
\end{aligned}
$$

The coefficients 0.260 and 0.0326 in equations (1) and (3) are close to the values 0.232 and 0.032 estimated by Helou et al. (2004). Some stars (e.g., cool red giants and asymptotic giant branch stars) have circumstellar dust that is in many cases warm enough to radiate strongly at wavelengths from 8 to $30 \mu \mathrm{m}$. If such stars are sufficiently numerous, the result will be a "stellar" contribution to the 8,16 , and $24 \mu \mathrm{m}$ fluxes that will be larger than assumed in 


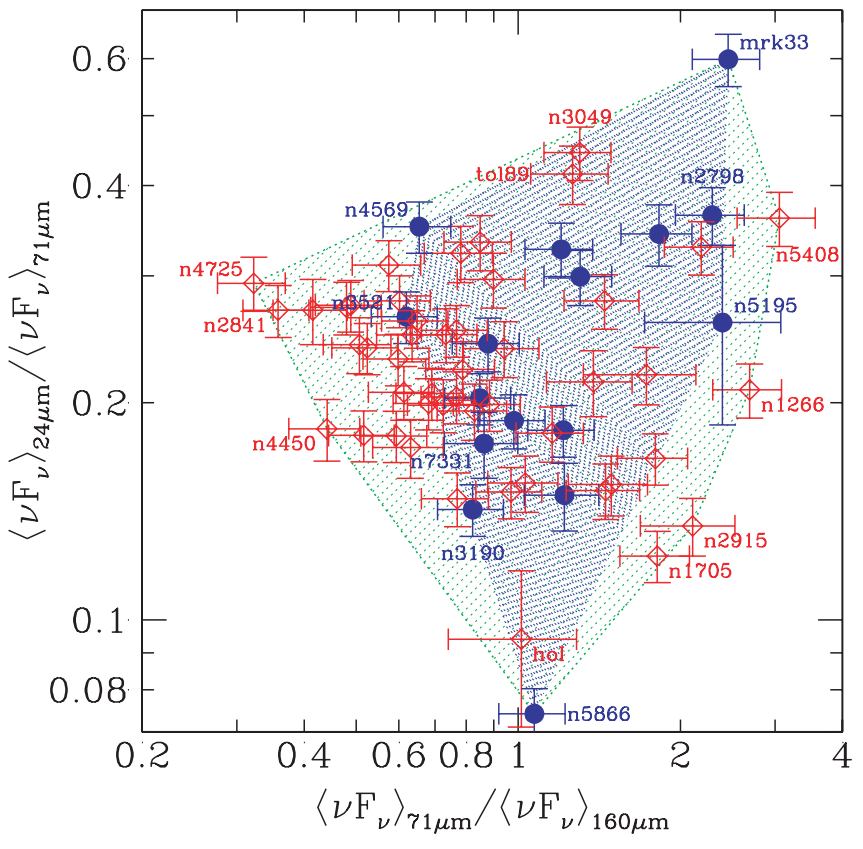

FIG. 1.- MIPS color-color plot for the 65 galaxies. The blue shaded area shows the region sampled by the 17 galaxies for which global SCUBA fluxes are known (plotted as blue filled circles); these only partially cover the color-color space populated by the SINGS sample (green shaded area). Galaxies without SCUBA fluxes are shown in red. Galaxies with relatively extreme flux ratios are labeled.

equations (1)-(3). In the absence of extinction at $3.6 \mu \mathrm{m}$, equations (1)-(3) will therefore overestimate the flux contributed by interstellar dust.

\subsection{IRS $16 \mu \mathrm{m}$}

In addition to IRAC and MIPS photometry, nine of the SINGS galaxies have been imaged using the IRS "blue peak-up" detector array, with nominal wavelength $\lambda=16 \mu \mathrm{m}$. The IRS blue peak-up observations were taken as part of the IRS instrument calibration program and consist of a grid of peak-up images covering the full extent of each galaxy. The reduced images, processed by IRS pipeline version S14, were mosaicked using the MOPEX program (ver. 030106) to remove cosmic rays after subtraction of a custom dark image. This custom dark image was created using images taken beyond the visible extent of the galaxy. In some cases, the best dark subtraction was found to require allowing the dark image to vary with time. "Sky" (zodiacal light and Galactic cirrus) contributions were also removed by this custom dark subtraction step. The total $16 \mu \mathrm{m}$ flux was measured using the same methods used for the IRAC and MIPS fluxes (Dale et al. 2005). The measured global fluxes were then scaled down by $\sim 15 \%$, to account for a recent Spitzer Science Center calibration update $(\sim 10 \%)$ and the appropriate aperture correction factor $(\sim 5 \%)$. Fluxes for these 11 galaxies at 16 and $24 \mu \mathrm{m}$ are given in Table 1 . The flux density ratio $\left\langle F_{\nu}\right\rangle_{16} /\left\langle F_{\nu}\right\rangle_{24}$ varies considerably among these galaxies, from a low of $0.376 \pm$ 0.033 for NGC 7552 to a high of $1.21 \pm 0.12$ for NGC 3190 .

Figure $2 a$ shows $P_{16} \equiv\left\langle\nu F_{\nu}^{\mathrm{ns}}\right\rangle_{16} /\left(\left\langle\nu F_{\nu}\right\rangle_{71}+\left\langle\nu F_{\nu}\right\rangle_{160}\right)$ versus $P_{24} \equiv\left\langle\nu F_{\nu}^{\mathrm{ns}}\right\rangle_{24} /\left(\left\langle\nu F_{\nu}\right\rangle_{71}+\left\langle\nu F_{\nu}\right\rangle_{160}\right)$ for the 10 galaxies for which we have $16,24,71$, and $160 \mu$ m photometry, where $F_{\nu}^{\text {ns }}$ is the nonstellar contribution from equations (1)-(3).

The flux ratio $P_{16}$ remains essentially constant at $P_{16} \approx 0.112 \pm$ 0.017 , while $P_{24}$ varies by almost a factor of 3 , from 0.06 to 0.18 . This suggests that the $16 \mu \mathrm{m}$ flux is not emitted by the same grains as the $24 \mu \mathrm{m}$ flux. In the dust model of Draine \& Li (2007,
TABLE 1

Global Fluxes (Jy) in IRS $16 \mu$ m Peak-up Band

\begin{tabular}{|c|c|c|c|}
\hline Galaxy & $\begin{array}{c}\left\langle F_{\nu}\right\rangle_{16} \\
(\mathrm{Jy})\end{array}$ & $\begin{array}{c}\left\langle F_{\nu}\right\rangle_{24} \\
(\mathrm{Jy})\end{array}$ & $\left\langle F_{\nu}\right\rangle_{16} /\left\langle F_{\nu}\right\rangle_{24}$ \\
\hline NGC $0337 \ldots \ldots \ldots \ldots$ & $0.375 \pm 0.029$ & $0.678 \pm 0.028$ & $0.553 \pm 0.049$ \\
\hline NGC $3190 \ldots \ldots \ldots \ldots . . .$. & $0.322 \pm 0.030$ & $0.267 \pm 0.011$ & $1.21 \pm 0.12$ \\
\hline NGC $3351 \ldots \ldots \ldots \ldots . . .$. & $1.39 \pm 0.23$ & $2.58 \pm 0.12$ & $0.538 \pm 0.091$ \\
\hline NGC $3521 \ldots$. & $4.86 \pm 0.59$ & $5.51 \pm 0.22$ & $0.88 \pm 0.11$ \\
\hline NGC $3627 \ldots \ldots \ldots \ldots . . .$. & $4.92 \pm 0.58$ & $7.42 \pm 0.30$ & $0.663 \pm 0.082$ \\
\hline NGC $3938 \ldots \ldots \ldots \ldots . . .$. & $0.849 \pm 0.068$ & $1.087 \pm 0.045$ & $0.781 \pm 0.070$ \\
\hline NGC $4321 \ldots \ldots \ldots \ldots . .$. & $2.64 \pm 0.43$ & $3.34 \pm 0.13$ & $0.79 \pm 0.13$ \\
\hline NGC $4536 \ldots \ldots \ldots \ldots . .$. & $1.74 \pm 0.14$ & $3.46 \pm 0.14$ & $0.503 \pm 0.044$ \\
\hline NGC $5055 \ldots \ldots \ldots \ldots . .$. & $5.09 \pm 0.43$ & $5.73 \pm 0.23$ & $0.888 \pm 0.083$ \\
\hline NGC $6946 \ldots \ldots \ldots . . . . .$. & $12.8 \pm 1.02$ & $20.37 \pm 0.81$ & $0.628 \pm 0.056$ \\
\hline NGC $7552 \ldots \ldots \ldots \ldots . .$. & $4.01 \pm 0.31$ & $10.66 \pm 0.44$ & $0.376 \pm 0.033$ \\
\hline
\end{tabular}

hereafter DL07), a minimum level of $24 \mu \mathrm{m}$ emission is produced by single-photon heating of PAHs or ultrasmall grains (with $\left\langle\nu L_{\nu}\right\rangle_{24} / L_{\mathrm{TIR}} \approx 0.03-0.06$, depending on $q_{\mathrm{PAH}}$; see Fig. 15 of DL07), but $P_{24}$ increases as the starlight intensity increases. Figure $2 a$ suggests that the $16 \mu \mathrm{m}$ emission may be dominated by single-photon heating of PAHs, remaining relatively constant even as $P_{24}$ rises due to increased starlight heating. This is supported by Figure $2 b$, which shows that the ratio of $7.9 \mu \mathrm{m}$ emission to $16 \mu \mathrm{m}$ emission is nearly constant for this sample, varying by only roughly $\pm 15 \%$ over the eight galaxies, consistent with the idea that both are primarily the result of single-photon heating of PAHs. This is consistent with the finding by Förster Schreiber et al. (2004) that, when averaged over disks of spiral galaxies, the $15 \mu \mathrm{m}$ continuum intensity is proportional to the PAH intensity in the $5-8 \mu \mathrm{m}$ region.

\subsection{SCUBA}

The dust properties in a galaxy will be best determined if submillimeter photometry is available to constrain the quantity of cool dust present. SCUBA observations at 450 and/or $850 \mu \mathrm{m}$ are available for 26 of the 65 galaxies, ${ }^{14}$ but not all of these are usable in the present study:

1. NGC 4254, NGC 4579, NGC 5194, and NGC 6946 were scan mapped, and the data processing removes an unknown amount of extended emission. Thus, global $850 \mu \mathrm{m}$ (or $450 \mu \mathrm{m}$ ) fluxes are not available for these galaxies. We restrict ourselves to data obtained in "jiggle map" mode, except for the scan-map observations of NGC 5195, for which background subtraction is thought to be more reliable because of the smaller angular extent of NGC 5195.

2. For some galaxies the SCUBA map did not fully cover the region where $160 \mu \mathrm{m}$ emission is detected. Dale et al. (2005) multiplied the observed SCUBA fluxes by a correction factor based on the fraction of the $160 \mu \mathrm{m}$ emission coming from the region mapped by SCUBA. Because the $850 \mu \mathrm{m} / 160 \mu \mathrm{m}$ flux ratio may be spatially variable, we choose here not to use the $850 \mu \mathrm{m}$ flux estimates for galaxies where the correction factor exceeds 1.6 (NGC 1097, NGC 4321, NGC 4736, and NGC 5033).

3. For the Sombrero galaxy, NGC 4594, the $850 \mu \mathrm{m}$ flux is dominated by the AGN (Bendo et al. 2006a), and the extranuclear $850 \mu \mathrm{m}$ flux is not reliably measured, so we cannot use the SCUBA data to constrain the dust model for this galaxy.

We thus have 17 galaxies where the dust models are constrained by $850 \mu \mathrm{m}$ SCUBA data (with $450 \mu \mathrm{m}$ fluxes for 3 of

${ }^{14}$ The 25 galaxies in Table 3 of Dale et al. (2005) plus NGC 1482. 

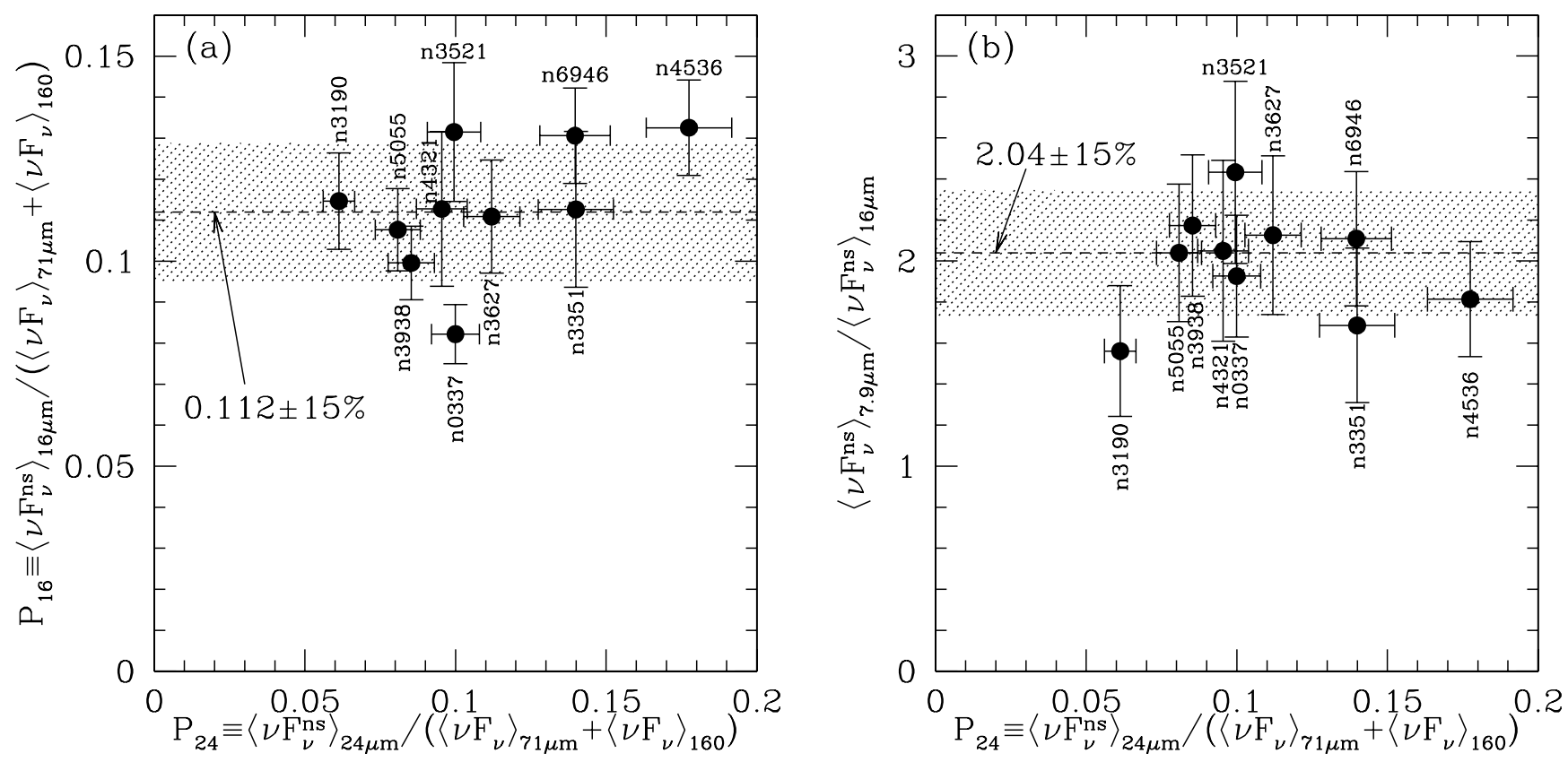

FIG. 2.- $(a) P_{16} \equiv\left\langle\nu F_{\nu}^{\text {ns }}\right\rangle_{16} /\left(\left\langle\nu F_{\nu}\right\rangle_{71}+\left\langle\nu F_{\nu}\right\rangle_{160}\right)$ vs. $P_{24} \equiv\left\langle\nu F_{\nu}^{\text {ns }}\right\rangle_{24} /\left(\left\langle\nu F_{\nu}\right\rangle_{71}+\left\langle\nu F_{\nu}\right\rangle_{160}\right)$ for the 10 galaxies imaged with the IRS $16 \mu$ m peak-up filter for which we also have global MIPS fluxes (NGC 7552 is excluded). (b) $\left\langle\nu F_{\nu}^{\text {ns }}\right\rangle_{79} /\left\langle\nu F_{\nu}^{\text {ns }}\right\rangle_{16}$ vs. $P_{24}$. Both the ratio of nonstellar $16 \mu$ m emission to total IR power and the ratio of nonstellar $7.9 \mu \mathrm{m}$ emission to nonstellar $16 \mu \mathrm{m}$ emission remain constant to within $\sim 15 \%$, even as $P_{24}$ varies by a factor of 3 . [See the electronic edition of the Journal for a color version of this figure.]

the 17) in addition to IRAC and MIPS data. The global fluxes $F_{\mathrm{obs}, b}$ and $1 \sigma$ uncertainties for each band $b$ are taken from Dale et al. (2007).

The 16 galaxies ${ }^{15}$ for which we have global SCUBA and MIPS photometry are indicated in Figure 1. While the galaxies with SCUBA photometry span a considerable portion of the populated region in MIPS color-color space, a number of the SINGS galaxies have MIPS colors that are outside of the range seen among the galaxies for which SCUBA data are available. For example, NGC 4725 and NGC 5408, with $\left\langle\nu F_{\nu}\right\rangle_{71 \mu \mathrm{m}} /\left\langle\nu F_{\nu}\right\rangle_{160 \mu \mathrm{m}}=0.33$ and 3.1, respectively, fall outside the range $0.6-2.4$ observed for this ratio among the SINGS-SCUBA galaxies. The dust models discussed below are able to reproduce the IRAC and MIPS fluxes for all of the SINGS galaxies. Galaxies such as NGC 4725 and NGC 2841, with the lowest values of $\left\langle\nu F_{\nu}\right\rangle_{71 \mu \mathrm{m}} /\left\langle\nu F_{\nu}\right\rangle_{160 \mu \mathrm{m}}$, require cooler dust (i.e., lower starlight intensities) than any of the SINGS-SCUBA galaxies. NCG 5408, with a very high value of $\left\langle\nu F_{\nu}\right\rangle_{71 \mu \mathrm{m}} /\left\langle\nu F_{\nu}\right\rangle_{160 \mu \mathrm{m}}$, requires hotter dust (higher starlight intensities). Submillimeter observations would be of great value to test the predicted submillimeter fluxes from these extreme cases.

\subsection{IRAS and ISO}

IRAS photometry is obtained from the latest SCANPI data for most SINGS sources and from HiRes maps for the largest SINGS targets (optical diameters $\gtrsim 10^{\prime}$ ), except in some bands (e.g., no IRAS $12 \mu \mathrm{m}$ flux for NGC 0024). A $20 \%$ calibration uncertainty estimate was applied. In the case of NGC 5195 (=M51b), the proximity of NCG 5194 (=M51a) made flux determination more difficult, and $50 \%$ uncertainty estimates were adopted.

The IRAS $25 \mu \mathrm{m}$ flux densities are not used, as they are superseded by the MIPS $24 \mu$ m photometry at essentially the same wavelength, but we employ the IRAS 12, 60, and $100 \mu \mathrm{m}$ photometry in our model fitting.

\footnotetext{
${ }^{15}$ NGC 7552 is absent from Fig. 1 because of detector saturation at $71 \mu \mathrm{m}$.
}

When available, we show ISOCAM 6.75 and $15 \mu \mathrm{m}$ photometry in the figures showing the SEDs, but they are not used in the model fitting.

\section{PHYSICAL DUST MODELS}

A direct estimate for the dust-to-hydrogen mass ratio $M_{\text {dust }} / M_{\mathrm{H}}$ in the local Milky Way (MW) can be obtained from the difference between total interstellar abundances (taken to be equal to current estimates for solar abundances) and observed gas-phase abundances in diffuse interstellar clouds, where the intensively studied diffuse cloud on the line of sight to $\zeta \mathrm{Oph}$ is taken to be representative. Using current estimates of solar abundances, this mass inventory results in $M_{\text {dust }} / M_{\mathrm{H}} \approx 0.0073$ (see Table 2).

We apply (and thereby also test) the dust models developed by Weingartner \& Draine (2001a, hereafter WD01) and Li \& Draine (2001, hereafter LD01) and updated by DL07. These dust models consist of specified mixtures of carbonaceous grains and amorphous silicate grains, with the smallest carbonaceous grains having the physical properties of PAH particles; the size distributions are chosen to reproduce the observed wavelength-dependent extinction in the local MW, in the Large Magellanic Cloud (LMC), and in the Small Magellanic Cloud (SMC) bar region (WD01). LD01 and Li \& Draine (2002) showed that these grain models appeared to be consistent with the observed IR emission from diffuse clouds in the MW and the SMC.

The DL07 dust models used here adopt PAH optical properties that are slightly different from those adopted by LD01. As discussed by DL07, the changes are in part to better conform to recent theoretical estimates for band strengths, and in part to improve agreement with band profiles determined observationally by Smith et al. (2007).

The PAH emission features at 3.3, 6.2, 7.7, 8.6, 11.3, 12.0, $12.7,16.4$, and $17 \mu \mathrm{m}$ are prominent in many galaxy spectra, and the dust models considered here include various abundances of PAHs; in each model the PAHs have a wide range of sizes. 
TABLE 2

Dust Mass per H from Milky Way Abundances

\begin{tabular}{|c|c|c|c|}
\hline$X$ & $\begin{array}{c}\left(N_{X} / N_{\mathrm{H}}\right)_{\odot}^{\mathrm{a}} \\
\quad(\mathrm{ppm})\end{array}$ & $\left(N_{X} / N_{\mathrm{H}}\right)_{\text {gas }} /\left(N_{X} / N_{\mathrm{H}}\right)_{\odot}^{\mathrm{a}}$ & $M_{X, \text { dust }} / M_{\mathrm{H}}$ \\
\hline С & 247 & 0.57 & 0.0013 \\
\hline N & 85 & 0.72 & 0.0003 \\
\hline О & 490 & 0.73 & 0.0021 \\
\hline $\operatorname{Mg}$ & 38 & 0.08 & 0.0008 \\
\hline 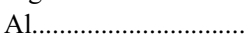 & $3^{\mathrm{b}}$ & $\lesssim 0.1^{\mathrm{c}}$ & 0.0001 \\
\hline $\mathrm{Si}$ & 32 & 0.05 & 0.0009 \\
\hline $\mathrm{Ca}$ & $2^{\mathrm{b}}$ & $0.0002^{\mathrm{d}}$ & 0.0001 \\
\hline Fe & 29 & 0.007 & 0.0016 \\
\hline $\mathrm{Ni}$ & 2 & 0.004 & 0.0001 \\
\hline Total …….................... & $\ldots$ & $\ldots$ & 0.0073 \\
\hline
\end{tabular}

\footnotetext{
a Jenkins (2004) except as noted.

${ }^{\mathrm{b}}\left(N_{X} / N_{\mathrm{H}}\right)$. from Grevesse \& Sauval (1998).

c Assumed.

d Savage \& Sembach (1996).
}

Because PAH emission is mainly the result of single-photon heating, the PAH emission spectrum depends on the size (heat capacity) of the PAH. The prominent $6.2,7.7$, and $8.6 \mu \mathrm{m}$ emission features are primarily due to PAHs with $\lesssim 10^{3} \mathrm{C}$ atoms (see Fig. 7 of DL07). Following DL07, we characterize the PAH abundance by a PAH index $q_{\mathrm{PAH}}$, defined to be the percentage of the total grain mass contributed by PAHs containing less than $10^{3} \mathrm{C}$ atoms:

$$
q_{\mathrm{PAH}} \equiv \frac{M\left(\text { PAHs with } N_{\mathrm{C}}<10^{3}\right)}{M_{\text {dust }}},
$$

where $M_{\text {dust }}$ includes the mass of all silicate and carbonaceous dust, including the PAHs.

WD01 put forward seven different size distributions for dust in the diffuse interstellar medium (ISM) of the MW. The models are all consistent with the average interstellar extinction law but have different $\mathrm{PAH}$ abundances, ranging from $q_{\mathrm{PAH}}=0.47 \%$ to 4.6\%. Some properties for these seven models are given in Table 3 , where the dust masses are calculated assuming densities of $3.5 \mathrm{~g} \mathrm{~cm}^{-3}$ for silicates and $2.24 \mathrm{~g} \mathrm{~cm}^{-3}$ for carbonaceous grains (WD01). The WD01 MW dust models have $M_{\text {dust }} / M_{\mathrm{H}} \approx 0.010$, about $40 \%$ larger than the value 0.0073 estimated from observed depletions (see Table 2), assuming solar abundances of $\mathrm{C}, \mathrm{Mg}$, $\mathrm{Si}$, and $\mathrm{Fe}$ in the local ISM. It is possible that current estimates of solar abundances of $\mathrm{C}, \mathrm{Mg}, \mathrm{Si}$, and $\mathrm{Fe}$ may be lower than the actual elemental abundances in the ISM today; a $\sim 25 \%$ increase in the solar abundances would bring $M_{\text {dust }} / M_{\mathrm{H}}$ estimated from depletions into accord with the WD01 model. Alternately, it is possible that the WD01 model overestimates the mass of interstellar dust by a factor of 1.4 or so.

The PAHs constitute the small-size end of the carbonaceous grain population; the varying PAH abundances are the result of varying the grain size distribution, while holding the overall extinction constant. The model with the largest PAH abundance appears to reproduce the observed IR-submillimeter emission from dust in the local ISM (LD01; DL07). Because it is likely that the PAH abundance varies from galaxy to galaxy, here we take linear combinations of these models to produce a sequence of $43 \mathrm{MW}$ models with $q_{\mathrm{PAH}}=0.4 \%-4.6 \%$ in steps of $0.1 \%$.

Similarly, we take linear combinations of the three WD01 models (as modified by DL07) for dust in the LMC2 region (with $q_{\mathrm{PAH}}=0.75 \%, 1.49 \%$, and $\left.2.37 \%\right)$ to produce a sequence of 18 LMC models with $q_{\mathrm{PAH}}=0.7 \%-2.4 \%$.
TABLE 3

Physical Dust Models

\begin{tabular}{|c|c|c|c|c|}
\hline$j_{M}$ & Model & $\begin{array}{c}q_{\mathrm{PAH}} \\
(\%)\end{array}$ & $M_{\text {dust }} / M_{\mathrm{H}}^{\mathrm{a}, \mathrm{b}}$ & $\begin{array}{c}A_{V} / N_{\mathrm{H}} \\
\left(\operatorname{mag~} \mathrm{cm}^{2} \mathrm{H}^{-1}\right)\end{array}$ \\
\hline 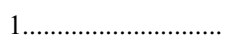 & MW3.1_00 & 0.47 & 0.0100 & $5.3 \times 10^{-22}$ \\
\hline ……….......... & MW3.1_10 & 1.12 & 0.0100 & $5.3 \times 10^{-22}$ \\
\hline 3...……………. & MW3.1_20 & 1.77 & 0.0101 & $5.3 \times 10^{-22}$ \\
\hline 4 & MW3.1_30 & 2.50 & 0.0102 & $5.3 \times 10^{-22}$ \\
\hline 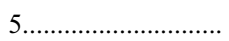 & MW3.1_40 & 3.19 & 0.0102 & $5.3 \times 10^{-22}$ \\
\hline 6................................. & MW3.1_50 & 3.90 & 0.0103 & $5.3 \times 10^{-22}$ \\
\hline ................. & MW3.1_60 & 4.58 & 0.0104 & $5.3 \times 10^{-22}$ \\
\hline 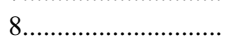 & LMC2_00 & 0.75 & 0.00343 & $1.2 \times 10^{-22}$ \\
\hline 9.…………….... & LMC2_05 & 1.51 & 0.00344 & $1.2 \times 10^{-22}$ \\
\hline $10 \ldots \ldots$ & LMC2_10 & 2.40 & 0.00359 & $1.2 \times 10^{-22}$ \\
\hline 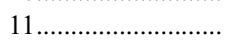 & SMCbar & 0.010 & 0.00206 & $6.2 \times 10^{-23}$ \\
\hline
\end{tabular}

${ }^{\mathrm{a}} M_{\mathrm{H}} \equiv M\left(\mathrm{H} \mathrm{I}+\mathrm{H}_{2}\right)$.

b $M_{\text {dust }} / M_{\text {gas }}=\left[M_{\text {dust }} / M_{\mathrm{H}}\right] / 1.36$.

The dust models include amorphous silicate grains. We assume optically thin dust when modeling the global SEDs, and therefore the models presented here do not include silicate absorption at $9.8 \mu \mathrm{m}$. Such absorption is in fact seen in observed spectra of the nuclei of a few of the galaxies in this sample (e.g., NGC 3198; Smith et al. 2007), but we neglect dust absorption in modeling the global emission.

The dust models employed here do not include $\mathrm{H}_{2} \mathrm{O}$ and other ices. $\mathrm{H}_{2} \mathrm{O}$ ice is known to be present in dark clouds in the $\mathrm{MW}$, in regions where $A_{V} \gtrsim 3.3 \mathrm{mag}$ (Whittet et al. 1988). In these regions, ices will modify the optical properties of the dust. However, it appears that such dark regions contain at most a modest fraction of the total dust in a galaxy; furthermore, the weak starlight in such regions implies that the dust grains located there radiate only a very small fraction of the total IR power. As seen below, an ice-free dust model appears to be satisfactory for modeling the IR emission from normal galaxies.

\section{CONSTRAINING THE DUST MODEL AND STARLIGHT INTENSITIES}

In addition to choosing a physical dust mixture (relative numbers of carbonaceous grains and silicate grains of different sizes, including the fraction $q_{\mathrm{PAH}}$ of the dust mass that is in PAHs), we must specify the intensity of the radiation that is heating the dust grains. Almost all of the heating of dust grains in a normal galaxy is from absorption of $h \nu<13.6 \mathrm{eV}$ photons. In a normal star-forming galaxy a significant (but subdominant) fraction of the stellar luminosity may be at $h \nu>13.6 \mathrm{eV}$, but most of these photons end up photoionizing hydrogen or helium rather than being absorbed by dust. The $h \nu<13.6 \mathrm{eV}$ recombination radiation (especially $\operatorname{Ly} \alpha$ ) may be largely absorbed by dust. However, the total power in ionizing photons is a minor fraction of the total stellar luminosity in a galaxy with a more or less steady rate of star formation. Only in galaxies that have undergone an extreme burst of star formation within the last $\sim 10^{7} \mathrm{yr}$ will the dust heating be dominated by Ly $\alpha$ and Lyman continuum radiation. Only in rare cases, e.g., NGC 1377 (Roussel et al. 2006), where the massive stars formed in a starburst are surrounded by dense, dusty $\mathrm{H}$ II regions, will the dust grains directly absorb a major fraction of the $h \nu>13.6 \mathrm{eV}$ radiation.

The IR emission from dust is relatively insensitive to the detailed spectrum of the $h \nu<13.6 \mathrm{eV}$ photons, and the DL07 models simply adopt the spectrum of the local interstellar radiation field (ISRF) as a reasonable representation for the average spectrum 
of the interstellar radiation in a normal spiral galaxy. The specific energy density of starlight is therefore taken to be

$$
u_{\nu}=U u_{\nu}^{(\mathrm{MMP} 83)},
$$

where $u_{\nu}^{\text {(MMP83) }}$ is the specific energy density estimated by Mathis et al. (1983) for the local Galactic ISRF and $U$ is a dimensionless scale factor. The intensity of interstellar UV radiation is often characterized by $G_{0}$, the ratio of the $6-13.6 \mathrm{eV}$ energy density relative to the value $5.288 \times 10^{-14} \mathrm{ergs} \mathrm{cm}^{-3}$ for the radiation field estimated by Habing (1968). The Mathis et al. (1983) ISRF has $u(0-13.6 \mathrm{eV}$ starlight $)=8.65 \times 10^{-13} \mathrm{ergs} \mathrm{cm}^{-3}$ and $u(6-$ $13.6 \mathrm{eV})=6.01 \times 10^{-14} \mathrm{ergs} \mathrm{cm}^{-3}$. Thus, $U=0.88 G_{0}$.

Dust at different locations in the galaxy will be exposed to different radiation intensities. A physically correct model would specify the locations of the stellar sources and the absorbing dust and would solve the radiative transfer problem (e.g., Tuffs et al. 2004), but this would require many uncertain assumptions about the relative distributions of stars and dust, as well as massive numerical computations.

Following DL07, we suppose that a large fraction of the dust in a galaxy is located in the diffuse ISM, exposed to starlight with an intensity that does not vary much within the galaxy. This "diffuse ISM" dust is idealized as being heated by a single radiation field, with intensity factor $U=U_{\min }$. In addition, DL07 suppose that a small fraction of the dust is located in regions where the radiation field is more intense, with a wide range of intensities ranging from $U=U_{\min }$ to $U=U_{\max } \gg U_{\min }$. This would include dust in PDRs, so we refer to this as the "PDR" component, and we use the power-law distribution function used by DL07. Although this PDR component contains only a small fraction of the total dust mass, in some galaxies it contributes a substantial fraction of the total power radiated by the dust. The models do not include a component corresponding to cold dust in dark clouds; as discussed in $\S 9.1$, such cold dust appears to account for a minor fraction of the dust mass and a very small fraction of the total dust luminosity. Thus, following DL07, we take the dust mass $d M_{\text {dust }}$ exposed to radiation intensities in $[U, U+d U]$ to be a combination of a $\delta$-function and a power law,

$$
\begin{aligned}
\frac{d M_{\text {dust }}}{d U}= & (1-\gamma) M_{\text {dust }} \delta\left(U-U_{\min }\right) \\
& +\gamma M_{\text {dust }} \frac{(\alpha-1)}{U_{\min }^{1-\alpha}-U_{\max }^{1-\alpha}} U^{-\alpha}
\end{aligned}
$$

( $\left.U_{\min } \leq U \leq U_{\max }, \alpha \neq 1\right)$, where $\delta$ is the Dirac $\delta$-function. This distribution function has five parameters: the total dust mass $M_{\text {dust }}$, the fraction $\gamma$ of the dust mass that is associated with the power-law part of the starlight intensity distribution, and three parameters $\left(U_{\min }, U_{\max }, \alpha\right)$ characterizing the distribution of starlight intensities in the high-intensity regions. The $\delta$-function term represents the fraction $(1-\gamma)$ of the dust that is heated by a general diffuse ISRF with approximately uniform intensity $\sim U_{\min }$. The power-law part of the distribution function, introduced by Dale et al. (2001) and used by Li \& Draine (2002), represents the fraction $\gamma$ of the dust mass that is close to OB associations and is exposed to radiation with intensity $U>U_{\min }$; this includes the dust in PDRs, where radiation intensities can be orders of magnitude higher than the "average" dust grain is exposed to. Equation (6) is a very simplistic description of the distribution of dust grain environments; it neglects the possibility that the dust properties (composition, size distribution) might be variable and correlated with $U$, and it also neglects regional variations in the spectrum of the starlight irradiating the dust. Despite its simplicity, we see here that equation (6) appears to capture the essential elements of the distribution of starlight intensities found in galaxies, yielding models with SEDs that approximate what is observed.

Very small particles cool almost completely between photon absorptions, and therefore the shape of the emission spectrum from these grains is almost independent of $U$. The larger grains, which account for more than half of the total absorption of starlight in the MW, do not cool significantly between photon absorptions, and their temperature depends on the intensity $U$. The observed FIR emission spectrum is sensitive to the temperature of these larger grains and therefore allows us to constrain the distribution function for $U$. While Spitzer provides unprecedented sensitivity to FIR emission, the spectral coverage is somewhat sparse, with a factor of 3 gap in wavelength between the IRAC $7.9 \mu \mathrm{m}$ and MIPS $24 \mu \mathrm{m}$ bands and a factor of 2.9 gap between the MIPS 24 and $71 \mu \mathrm{m}$ bands. The IRS "peak-up" mode observations can be used for imaging at 16 and $26 \mu \mathrm{m}$, but these observations are not generally available. The large gaps in wavelength coverage leave considerable uncertainty in the SED at wavelengths between the bands.$^{16}$ Furthermore, the longest wavelength band, at $160 \mu \mathrm{m}$, falls on the short-wavelength side of the emission peak for dust temperatures $T_{d} \lesssim(1 / 6) h c /(160 \mu \mathrm{m})=15 \mathrm{~K}$. As a result, Spitzer photometry does not strongly constrain the abundance of dust with temperatures $T_{d} \lesssim 15 \mathrm{~K}$. To determine the amount of cool dust, global submillimeter observations are required. Fortunately, such data exist for 17 of the 65 galaxies for which we have complete Spitzer photometry (or near complete in the case of NGC 7552), and this subsample is analyzed first, in $\S 5$.

We select a model for the IR emission from a galaxy as follows. For each size and composition of dust, we precompute and tabulate the temperature distribution function $d P / d T$ for the dust grain illuminated by the radiation field of equation (5) for selected values of $U$ (with $0.1 \leq U \leq 10^{7}$ ), using heat capacities, absorption cross sections, and numerical methods described by Draine \& Li (2001), LD01, and DL07. Neutral and ionized PAHs are assumed to have different absorption cross sections. The ionized fraction $\phi_{i}\left(N_{\mathrm{C}}\right)$ (a function of the number $N_{\mathrm{C}}$ of carbon atoms in the PAH) will depend primarily on the ratio $U \sqrt{T_{e}} / n_{e}$, where $n_{e}$ and $T_{e}$ are the electron density and temperature (Bakes \& Tielens 1994; Weingartner \& Draine 2001b). Here we take the ionized fraction $\phi_{i}\left(N_{\mathrm{C}}\right)$ estimated by LD01 for the local diffuse $\mathrm{ISM}^{17}$ [we are therefore implicitly assuming that other galaxies have the same mix of $U \sqrt{T_{e}} / n_{e}$ as the MW]. Our model-fitting procedure assumes that all galaxies have the same shape for the PAH size distribution, the same PAH ionized fraction $\phi_{i}\left(N_{\mathrm{C}}\right)$, and the same spectral shape of the illuminating starlight. As a result, our model fits have fixed ratios of PAH emission band strengths. Observations do reveal variations in PAH band ratios from one galaxy to the next—for example, $L(7.7 \mu \mathrm{m}) / L(11.3 \mu \mathrm{m})$ varies from $\sim 4-5$ to $\lesssim 0.7$ as we progress from $\mathrm{H}_{\text {II }}$-dominated nuclei to AGN-dominated nuclei (Smith et al. 2007), and low values of $L(7.7 \mu \mathrm{m}) / L(11.3 \mu \mathrm{m})$ are also seen in some Galactic and AGN-free extragalactic regions (e.g., Reach et al. 2000; Hony et al. 2001; Pagani et al. 1999) — but most spiral galaxies

\footnotetext{
16 In principle, the IRS instrument can provide 6-36 $\mu \mathrm{m}$ photometry, and the MIPS SED mode covers 55-95 $\mu \mathrm{m}$, but observing time considerations preclude using these observing modes for large areas.

17 Following LD01, we assume $43 \%$ of the dust to be in the "cold neutral medium" (CNM; $\left.n_{e} / U \approx 0.045 \mathrm{~cm}^{-3}, T_{e} \approx 100 \mathrm{~K}\right), 43 \%$ to be in the "warm neutral medium" (WNM; $n_{e} / U \approx 0.04 \mathrm{~cm}^{-3}, T_{e} \approx 6000 \mathrm{~K}$ ), and $14 \%$ to be in the "warm ionized medium" (WIM; $n_{e} / U \approx 0.1 \mathrm{~cm}^{-3}, T_{e} \approx 8000 \mathrm{~K}$ ). The resulting $\phi_{i}\left(N_{\mathrm{C}}\right)$ is shown in Fig. 7 of LD01 and Fig. 8 of DL07.
} 
have global PAH feature ratios that are near average and characteristic of star-forming regions.

With these temperature distribution functions for the dust and the dust absorption cross section $C_{\text {abs }}(\lambda)$, we can calculate the time-averaged IR emission for a given grain type and size for each of the discrete values of $U$ considered; we then sum over the grain types and sizes for a dust model $j_{M}$ (see Table 3 ) to find the power radiated per unit frequency per unit mass of dust mixture $j_{M}$ exposed to starlight intensity $U$ :

$$
\begin{aligned}
p_{\nu}^{(0)}\left(j_{M}, U\right)= & \frac{1}{M\left(j_{M}\right)} \sum_{k} \int d a\left(\frac{d n_{k}}{d a}\right)_{j_{M}} \\
& \times \int d T \frac{d P(k, a)}{d T} 4 \pi B_{\nu}(T) C_{\mathrm{abs}}(a, \lambda), \\
M\left(j_{M}\right) \equiv & \sum_{k} \int d a\left(\frac{d n_{k}}{d a}\right)_{j_{M}} \frac{4 \pi a^{3}}{3} \rho_{k}
\end{aligned}
$$

where the sum runs over the different grain types $k$ (amorphous silicate, graphite, $\mathrm{PAH}^{0}, \mathrm{PAH}^{+}$) in the DL07 dust model, with $\rho_{k}$ the density of material $k$ and $n_{k}(a)$ being the number of dust grains of type $k$ per $\mathrm{H}$ nucleon, with radii $\leq a$.

We also calculate the specific power per unit dust mass

$$
\begin{aligned}
p_{\nu}\left(j_{M}, U_{\min }, U_{\max }, \alpha\right) \equiv & \frac{(\alpha-1)}{U_{\min }^{1-\alpha}-U_{\max }^{1-\alpha}} \\
& \times \int_{U_{\min }}^{U_{\max }} p_{\nu}^{(0)}\left(j_{M}, U\right) U^{-\alpha} d U
\end{aligned}
$$

for a power-law distribution of starlight intensities, $d M_{\text {dust }} / d U \propto$ $U^{-\alpha}$. For each dust model $j_{M}$ we consider a range of values of $U_{\min }$ and $U_{\max }$.

At wavelengths $\lambda \lesssim 5 \mu \mathrm{m}$, the emission from a galaxy is dominated by starlight. For $\lambda \geq 3.6 \mu \mathrm{m}$ we neglect reddening and approximate the stellar contribution by a dilute blackbody with color temperature $T_{\star}=5000 \mathrm{~K}$, which Smith et al. (2007) found to provide a suitable approximation to the stellar continuum at $\lambda>5 \mu \mathrm{m}$. The model emission spectrum is then

$$
\begin{aligned}
F_{\nu, \text { model }}= & \Omega_{\star} B_{\nu}\left(T_{\star}\right)+\frac{M_{\text {dust }}}{4 \pi D^{2}}\left[(1-\gamma) p_{\nu}^{(0)}\left(j_{M}, U_{\text {min }}\right)\right. \\
& \left.+\gamma p_{\nu}\left(j_{M}, U_{\min }, U_{\max }, \alpha\right)\right],
\end{aligned}
$$

where $\Omega_{\star}$ is the solid angle subtended by the stars. For each $\left(j_{M}, U_{\min }, U_{\max }, \alpha\right)$, we obtain $\Omega_{\star}, M_{\text {dust }}$, and $\gamma$ by minimizing

$$
\chi^{2} \equiv \sum_{b} \frac{\left(F_{\mathrm{obs}, b}-\left\langle F_{\nu, \text { model }}\right\rangle_{b}\right)^{2}}{\sigma_{\mathrm{obs}, b}^{2}+\sigma_{\text {model }, b}^{2}},
$$

where the sum is over observed bands $b,\left\langle F_{\nu, \text { model }}\right\rangle_{b}$ is the model spectrum convolved with the response function for band $b$, and $\sigma_{\mathrm{obs}, b}$ is the observational uncertainty in the observed flux density $F_{\mathrm{obs}, b}$ for band $b$. The ad hoc insertion of the $\sigma_{\text {model }, b}^{2}$ term in the denominator in equation (11) is to allow for the approximate nature of our models and is motivated by the very high photometric accuracy achieved for the MIPS $24 \mu \mathrm{m}$ photometry, with $\sigma_{\mathrm{obs}, 24 \mu \mathrm{m}} / F_{\mathrm{obs}, 24 \mu \mathrm{m}}=0.040$ achieved for many of the SINGS galaxies. We do not want the fitting procedure to give extreme weight to one or two bands where $F_{\mathrm{obs}, b}$ may have been mea- sured with very high precision (i.e., small $\sigma_{\mathrm{obs}, b} / F_{\mathrm{obs}, b}$ ) because we do not expect our models to fit perfectly in the limit of perfect observations. Our subjective sense is that the present models should be regarded as satisfactory if they are able to reproduce the actual fluxes (i.e., perfect data, with $\sigma_{\mathrm{obs}, b}=0$ ) to within, say, $\pm 10 \%$. We therefore arbitrarily set $\sigma_{\text {model }, b}=0.1\left\langle F_{\nu, \text { model }}\right\rangle_{b}$ for each band $b$. It is important to recognize that equation (11) differs from the usual $\chi^{2}$ used for testing models, and the numerical values of $\chi^{2}$ found here are not rigorous measures of the goodness of fit. Nevertheless, these $\chi^{2}$ values do appear to provide a practical guide to distinguish between good fits and poor fits.

The model components must be nonnegative: we require $\Omega_{*} \geq 0$, $M_{\text {dust }} \geq 0$, and $0 \leq \gamma \leq 1$. We select the dust model $j_{M}$ and intensity model $\left(\gamma, \bar{U}_{\min }, U_{\max }, \alpha\right)$ giving the best fit (smallest $\left.\chi^{2}\right)$ subject to these constraints.

For the MW dust models, there are effectively seven adjustable parameters: $\Omega_{\star}, M_{\text {dust }}, q_{\text {PAH }}\left(\right.$ via $\left._{M}\right), \gamma, U_{\text {min }}, U_{\text {max }}$, and $\alpha$. However, we fix one of these parameters from the outset, by setting $\alpha=2$. This choice is based on experimentation that indicated that the quality of the fit is not very sensitive to the precise value of $\alpha$, with $\alpha \approx 2$ providing satisfactory fits for diverse galaxies. Therefore, we start with six parameters to be adjusted. For the galaxies with SCUBA data, we examine the effects of varying $U_{\max }$, but we see in $\S 5.4 .2$ that setting $U_{\max }=10^{6}$ works well; therefore, the number of adjustable parameters is, effectively, five: $\Omega_{\star}, M_{\text {dust }}, q_{\mathrm{PAH}}, U_{\text {min }}$, and $\gamma$. If the number of observed bands is $N_{b}$, the number of degrees of freedom equals $N_{b}-5$, where $N_{b}$ ranges from $N_{b}=9$ (e.g., NGC 0024, with four IRAC bands, three MIPS bands, and IRAS 60 and $100 \mu \mathrm{m}$ ) to $N_{b}=12$ (e.g., NGC 3190, with four IRAC bands, three MIPS bands, IRS $16 \mu \mathrm{m}, I R A S 12,60$, and $100 \mu \mathrm{m}$, and SCUBA $850 \mu \mathrm{m})$. To assess the goodness of fit, we calculate $\chi^{2}$ per degree of freedom:

$$
\chi_{r}^{2} \equiv \frac{\chi^{2}}{N_{b}-5} .
$$

\section{SCUBA-CONSTRAINED DUST MODELS}

\subsection{Spectral Energy Distributions}

The distribution of grain temperatures in a dust cloud or a galaxy is never determined precisely. At wavelength $\lambda$ the thermal emission per unit dust mass $\propto\left(e^{h c / \lambda k T_{d}}-1\right)^{-1}$. For $\lambda \lesssim$ $360 \mu \mathrm{m}\left(20 \mathrm{~K} / T_{d}\right)$, small errors in the adopted $T_{d}$ lead to large errors in the dust mass inferred from the observed $F_{\nu}$. For example, varying the adopted $T_{d}$ from 22 to $18 \mathrm{~K}$ produces a $150 \%$ increase in the mass of dust required to produce a given $160 \mu \mathrm{m}$ flux, but only a $35 \%$ increase in the mass required to produce a given $850 \mu \mathrm{m}$ flux. Observations at $\lambda \gtrsim 450 \mu \mathrm{m}$ provide dust masses with the least sensitivity to dust temperature (for dust temperatures $T_{d} \gtrsim 15 \mathrm{~K}$ ). Therefore, the subset of 17 SINGSSCUBA galaxies will be those for which we can best determine the dust mass.

For each galaxy Table 4 lists the best-fit model for $10^{3} \leq$ $U_{\max } \leq 10^{7}$, for fixed $\alpha=2$. If the best-fit model has either LMC or SMC dust models (see NGC 5713), then we also list the parameters for the best-fit model using MW dust. Finally, if the best-fit MW dust model has $U_{\max } \neq 10^{6}$, we also list the parameters for the best-fit MW dust model with $U_{\max }=10^{6}$.

The models are generally very successful in fitting the observed SEDs. The SED of NGC 3190, together with a model fit, appears in Figure $3 a$. This is an example where the model (with $U_{\min }=2.5, U_{\max }=10^{7}, \gamma=1.5 \times 10^{-3}$ ) provides an excellent fit to the observations, with $\chi_{r}^{2}=0.38$. The inferred dust mass is 
TABLE 4

17 SiNGS Galaxies with IRAC, MIPS, and SCUBA Global Fluxes

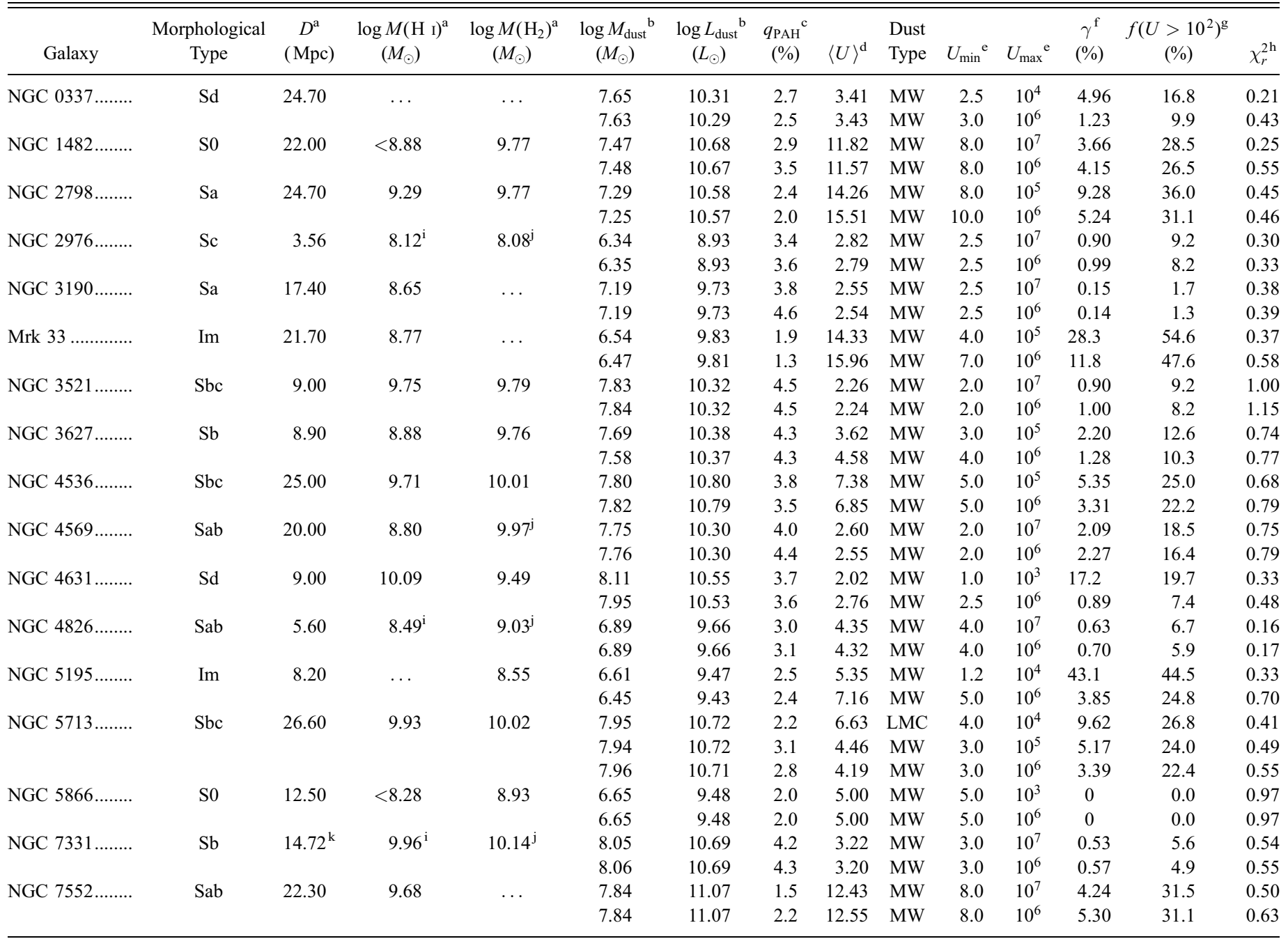

${ }^{a} D, 21 \mathrm{~cm}$ flux, and CO 1-0 flux from Kennicutt et al. (2003) unless otherwise noted.

b Estimated from dust model.

${ }^{c}$ Fraction of dust mass contributed by PAHs with $N_{\mathrm{C}}<10^{3} \mathrm{C}$ atoms.

d Dust-weighted mean starlight intensity scale factor.

e $\left(U_{\min }, U_{\max }\right)=$ (lower, upper) cutoff for starlight intensity scale factor $U$.

${ }^{\mathrm{f}}$ Fraction of dust mass in regions with $U>U_{\min }$.

${ }^{g}$ Fraction of dust luminosity from regions with $U>10^{2}$.

${ }^{\mathrm{h}} \chi^{2} /\left(N_{b}-5\right)$, where $N_{b}$ is the number of bands used for fitting.

i $21 \mathrm{~cm}$ flux from F. Walter (2005, private communication).

j CO 1-0 flux from Sheth et al. (2005).

${ }^{\mathrm{k}}$ Freedman et al. (2001).

$M_{\text {dust }}=10^{7.19} M_{\odot}$, and the mean starlight intensity is estimated to be $\langle U\rangle=2$.6. Only a small fraction $\gamma \approx 0.0015$ of the dust is in regions with starlight intensities $U>U_{\min }$. Also shown in Figure $3 a$ (solid line) is a fit with the upper cutoff fixed at $U_{\max }=$ $10^{6}$. The model SED is nearly identical (the solid line and dotdashed lines coincide in the plot), and the derived dust mass is unchanged.

Figure $3 b$ shows the SED of NGC 2798, a galaxy where the dust is considerably warmer than in NGC 3190 . The best-fit dust $\operatorname{model}\left(U_{\min }=8, U_{\max }=10^{5}, \gamma=0.09\right)$ provides a good fit $\left(\chi_{r}^{2}=\right.$ 0.45 ) to the observations, but the model with $U_{\max }=10^{6}$ provides nearly as good a fit $\left(\chi_{r}^{2}=0.46\right)$, a derived dust mass differing by only 0.04 dex, and similar $q_{\mathrm{PAH}}(2.0 \%$ vs. $2.5 \%)$.

The model fits to NGC 2798 both have a noticeable "bump" near $\sim 33 \mu \mathrm{m}$, produced by the warm graphite grains in the DL07 model. The small but nonzero conductivity for $\boldsymbol{E} \| c$ in graphite ${ }^{18}$ causes the absorption cross section $C_{\text {abs }}$ to rise to a broad local maximum near $\sim 33 \mu \mathrm{m}$ (Draine \& Lee 1984; DL07). When exposed to starlight with $U \gtrsim 10^{4}$, graphite grains produce a broad emission bump peaking in the $30-35 \mu \mathrm{m}$ region, depending on $U$. Unfortunately, the MIPS 24 and $71 \mu \mathrm{m}$ bands straddle this feature and do not constrain it. Implications of the model $33 \mu \mathrm{m}$ feature are discussed in $\S 9.3$.

\subsection{Dust-to-Gas Ratio}

If the interstellar abundances of carbon and all heavier elements were proportional to the gas-phase oxygen abundance,

\footnotetext{
18 The $c$-axis is normal to the sheets of hexagonally organized $\mathrm{C}$ atoms.
} 


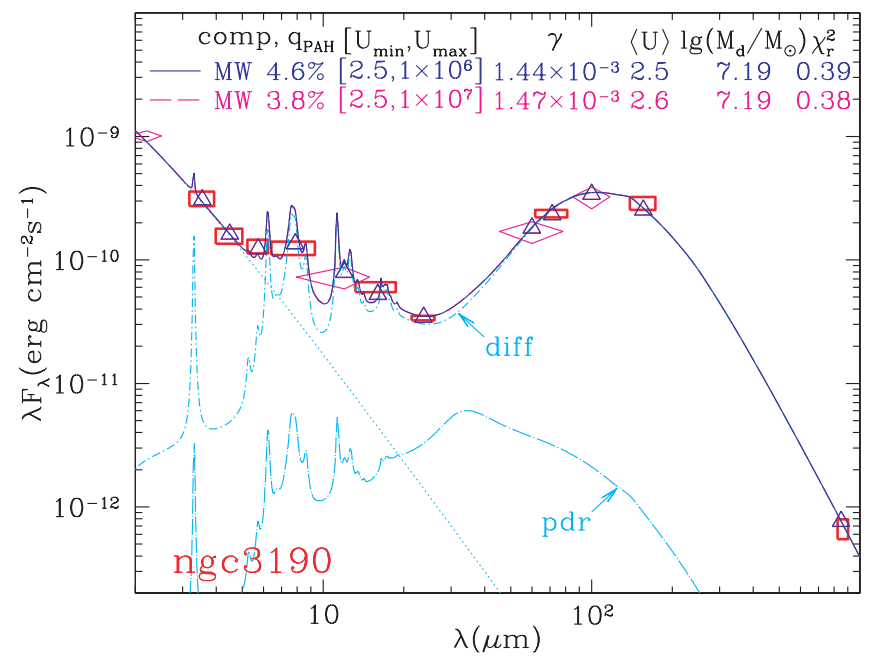

FIG. $3 a$

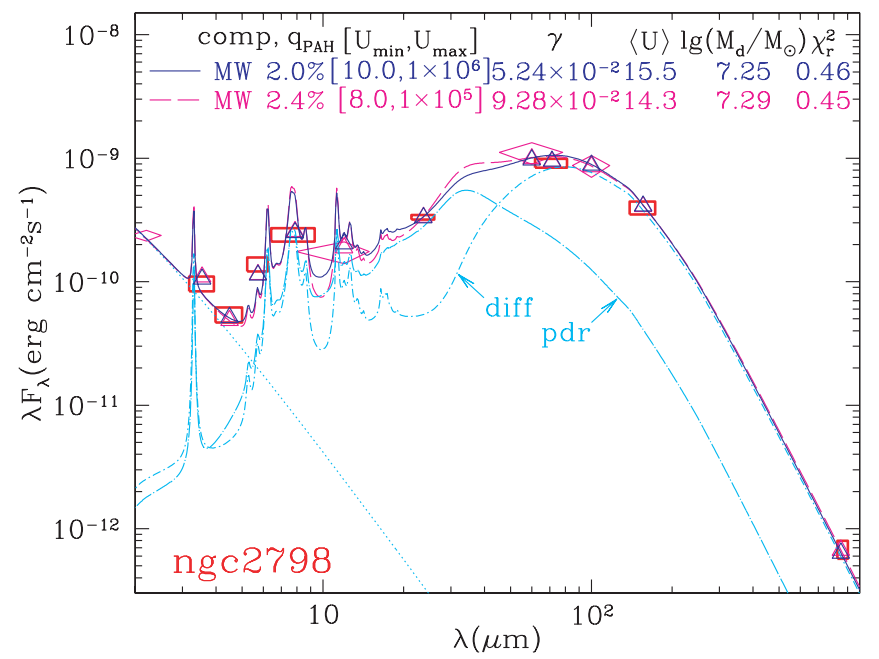

FIG. $3 b$

FIG. 3.- (a) SED for the SAap galaxy NGC 3190. Rectangles show observed fluxes in the IRAC, MIPS, SCUBA, and IRS $16 \mu$ m bands; diamonds show 2 MASS $2.2 \mu$ m and IRAS 12, 60, and $100 \mu \mathrm{m}$ fluxes. Vertical extent of rectangles and diamonds corresponds to $\pm 1 \sigma$ range, and width corresponds to nominal width of band. The solid line shows the best-fit $U_{\max }=10^{6}$ model, with IRAC, MIPS, IRS $16 \mu \mathrm{m}$, IRAS, and SCUBA data used to constrain the fit; $N_{b}=12$ for NGC 3190 . Triangles show the model convolved with the IRAC, MIPS, IRS $16 \mu \mathrm{m}, I R A S$, and SCUBA bands. Dot-dashed lines show separate contributions of starlight and emission from dust heated by $U=U_{\text {min }}$ (labeled "diff") and dust heated by $U_{\min }<U<U_{\max }$ (labeled "pdr"). The long-dashed line shows the best-fit model when $U_{\max }$ is unconstrained; for this case the model spectrum is nearly indistinguishable for the simple reason that there is relatively little dust $(\gamma \approx 0.0015)$, and therefore relatively little power, in the "pdr" component. (b) Same as (a), but for the SBa galaxy NGC 2798, with $N_{b}=11$ (global imaging in the IRS $16 \mu \mathrm{m}$ band is unavailable).

and if the same fraction of the major condensable elements $(\mathrm{C}$, $\mathrm{O}, \mathrm{Mg}, \mathrm{Si}, \mathrm{Fe}$ ) were in solid form as in the $\mathrm{MW}$, then all galaxies would be expected to conform to

$$
\frac{M_{\text {dust }}}{M_{\mathrm{H}}} \approx 0.010 \frac{(\mathrm{O} / \mathrm{H})}{(\mathrm{O} / \mathrm{H})_{\mathrm{MW}}}
$$

where $(\mathrm{O} / \mathrm{H})_{\mathrm{MW}}$ is the oxygen abundance in the local MW and the factor 0.010 is from the MW dust models in Table 3.

Table 4 gives the estimated dust mass $M_{\text {dust }}$ for the 17 galaxies. For many of the galaxies there are measurements of $21 \mathrm{~cm}$ emission and $\mathrm{CO}$ emission from which the masses of $\mathrm{H} \mathrm{I}$ and $\mathrm{H}_{2}$ can be estimated (see Table 4). The usual assumption is made that the $\mathrm{H}_{2}$ mass is proportional to the $\mathrm{CO} 1-0$ luminosity:

$$
\begin{aligned}
M\left(\mathrm{H}_{2}\right)= & 1.57 \times 10^{4} M_{\odot}\left[\frac{X_{\mathrm{CO}}}{4 \times 10^{20} \mathrm{~cm}^{-2}\left(\mathrm{~K} \mathrm{~km} \mathrm{~s}^{-1}\right)^{-1}}\right] \\
& \times\left(\frac{D}{\mathrm{Mpc}}\right)^{2}\left(\frac{S_{\mathrm{CO}}}{\mathrm{Jy} \mathrm{km} \mathrm{s}^{-1}}\right),
\end{aligned}
$$

where $X_{\mathrm{CO}}$ is the ratio of $\mathrm{H}_{2}$ column density to antenna temperature integrated over the CO 1-0 line, and $S_{\mathrm{CO}} \equiv \lambda_{1-0} \int F_{\nu}(1-0) d \nu$, where $\int F_{\nu}(1-0) d \nu$ is the $\mathrm{CO} 1-0$ line flux. Use of the so-called CO 1-0 luminosity to estimate $\mathrm{H}_{2}$ masses remains suspect, and the value to use for the proportionality constant $X_{\mathrm{CO}}$ is uncertain, with estimates for the MW ranging from $3.0 \times$ $10^{20} \mathrm{~cm}^{-2}\left(\mathrm{~K} \mathrm{~km} \mathrm{~s}^{-1}\right)^{-1}$ (Young \& Scoville 1991) to $1.56 \times$ $10^{20} \mathrm{~cm}^{-2}\left(\mathrm{~K} \mathrm{~km} \mathrm{~s}^{-1}\right)^{-1}$ (Hunter et al. 1997). Here we use the value $X_{\mathrm{CO}}=4 \times 10^{20} \mathrm{~cm}^{-2}\left(\mathrm{~K} \mathrm{~km} \mathrm{~s}^{-1}\right)^{-1}$, the value recommended by Blitz et al. (2007) for giant molecular clouds (GMCs) in Local Group galaxies. We discuss the value of $X_{\mathrm{CO}}$ in $\S 9.4$, where we argue that dust mass estimates for the SINGS galaxies favor $X_{\mathrm{CO}}=4 \times 10^{20} \mathrm{~cm}^{-2}\left(\mathrm{~K} \mathrm{~km} \mathrm{~s}^{-1}\right)^{-1}$.

Figure 4 shows the dust/hydrogen mass ratio for the 12 SINGSSCUBA galaxies for which the total (atomic and molecular) gas masses are known. The derived dust-to-gas ratios are tightly clustered, with median $M_{\text {dust }} / M_{\mathrm{H}} \approx 0.005$. The lowest dust-togas ratio is for NGC 2798 , with $M_{\text {dust }} / M_{\mathrm{H}} \approx 0.0023$; the highest is for NGC 2976, with $M_{\text {dust }} / M_{\mathrm{H}} \approx 0.0089$.

The dust-to-gas mass ratios in Figure 4 are broadly comparable to the value $\sim 0.007$ estimated from gas-phase abundances in the local MW (Table 2) or 0.010 estimated from models for the observed MW extinction (Table 3 ). The observed range $0.003 \lesssim$ $M_{\text {dust }} / M_{\mathrm{H}} \lesssim 0.01$ is consistent with galactic metallicities between $\sim 0.3$ and $\sim 1$ times solar, if a similar fraction of the heavy elements is in the form of dust as in the MW.

Based on Figure 4, we conclude that the model-fitting procedure provides dust mass estimates for this sample of galaxies that are in reasonable agreement with the dust masses expected if depletions are similar to depletions in the MW. Furthermore, the model appears to be able to reproduce the observed emission spectrum (see, e.g., Fig. 3). The metallicity dependence of the dust-to-gas ratio is examined in $\S 7.1$.

\subsection{Role of LMC and SMC Dust Models}

We now ask whether it is necessary to use the LMC and SMC dust models in the determination of dust masses: do the dust models developed for the LMC and SMC lead to improved fits to the observed spectra, and, if so, how different are the inferred dust masses and PAH abundances? For each galaxy in the SINGSSCUBA subset, we searched for the model that minimized $\chi_{r}^{2}$; for 16 of the 17 galaxies, an MW dust model was preferred, for NGC 5713 an LMC dust model was favored, but in no case did the SMC bar dust model give the best fit. For NGC 5713, where an LMC dust model gave the smallest $\chi_{r}^{2}$, we also give in Table 4 the parameters for the best-fit MW model; the derived $M_{\text {dust }}$ differs from that obtained using the LMC model by only $0.01 \mathrm{dex}$, and $q_{\mathrm{PAH}}=3.1 \%$, versus $2.2 \%$ for the LMC model; when the MW model fit is constrained to have $U_{\max }=10^{6}$, the derived $q_{\mathrm{PAH}}=2.8 \%$. Therefore, at least for the 17 galaxies with SCUBA photometry, the model fitting can be limited to MW-type 


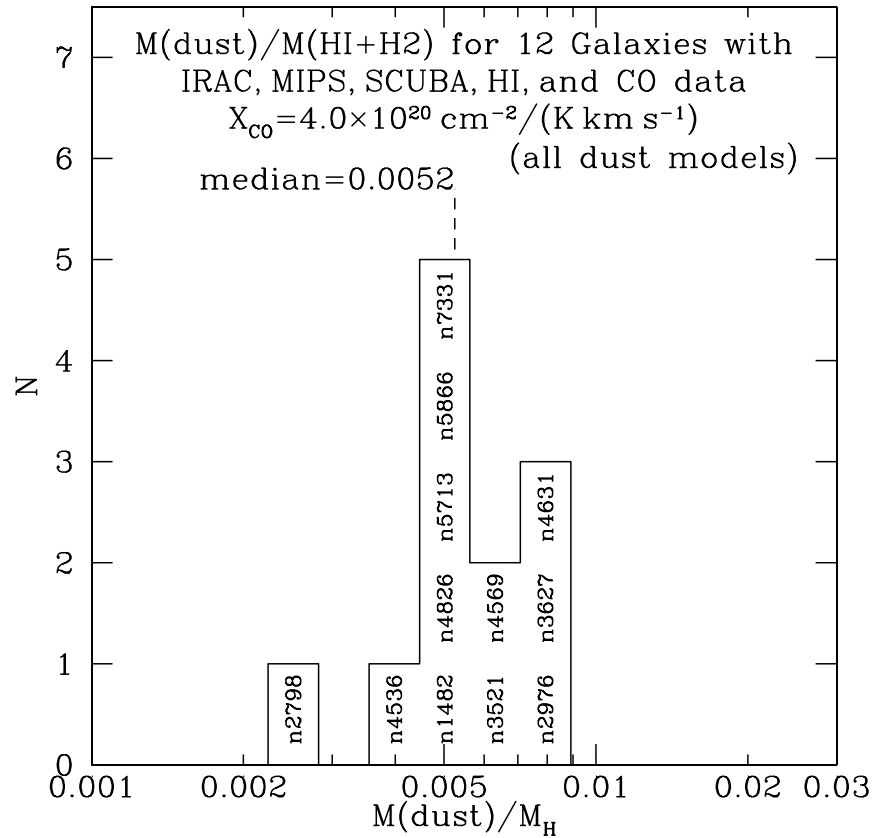

FIG. 4.- Histograms of $M_{\text {dust }} / M\left(\mathrm{H} \mathrm{I}_{\mathrm{I}}+\mathrm{H}_{2}\right)$ for the 12 galaxies in the sample for which both $\mathrm{H}_{\mathrm{I}} 21 \mathrm{~cm}$ and $\mathrm{CO} 1-0$ have been measured, using $X_{\mathrm{CO}}=4 \times$ $10^{20} \mathrm{~cm}^{-2}\left(\mathrm{~K} \mathrm{~km} \mathrm{~s}^{-1}\right)^{-1}$ to estimate $M\left(\mathrm{H}_{2}\right)$.

dust models with minimal impact on the inferred dust masses or PAH index.

In $\S 6$ we demonstrate that we can obtain good estimates for $M_{\text {dust }}$ and $q_{\text {PAH }}$ even for galaxies lacking submillimeter photometry, by limiting the search to models with $U_{\min } \geq 0.7$. Here we compare estimates for $q_{\mathrm{PAH}}$ and $M_{\text {dust }}$ obtained using LMC and SMC dust models with those obtained using MW dust models.

\subsubsection{PAH Abundance $q_{\mathrm{PAH}}$}

Let $q_{\mathrm{PAH}}(\mathrm{MW})$ be the best-fit estimates for $q_{\mathrm{PAH}}$ when fitting with only MW dust models, and let $q_{\mathrm{PAH}}(x \mathrm{MC})$ be the estimate obtained using only LMC+SMC dust models. As discussed below (see $\S 7.4$ ), we exclude four galaxies for which $q_{\mathrm{PAH}}$ cannot be reliably estimated. Because the LMC and SMC dust models are limited to $q_{\mathrm{PAH}} \leq 2.4 \%$, we further limit consideration to galaxies for which $q_{\mathrm{PAH}}(\mathrm{MW}) \leq 2.4 \%$. The MW models have $q_{\text {PAH }}(\mathrm{MW}) \geq 0.4 \%$. Therefore, for the seven galaxies where the best-fit MW model has $q_{\text {PAH }}=0.4 \%$, we can conclude only that the actual $q_{\text {PAH }} \leq 0.4 \%$.

As seen in Figure $5 a$, the best-fit MW models and the best-fit LMC+SMC models generally have similar values of $q_{\mathrm{PAH}}$ : $16 / 19$ galaxies have $\left|q_{\text {PAH }}(\mathrm{MW})-q_{\text {PAH }}(x \mathrm{MC})\right| \leq 0.4 \%$. There is one extreme outlier: the S0 galaxy NGC 5866. NGC 5866 has relatively little dust: the flux in IRAC band 3 is consistent with starlight alone, and there is only a modest excess due to dust in IRAC band 4 . As a result, $q_{\text {PAH }}$ is not very securely determined for NGC 5866. When the fitting is limited to LMC and SMC dust models, the best fit is produced by the SMC model, with $q_{\mathrm{PAH}}=$ $0.1 \%$. However, this fit, with $\chi_{r}^{2}=1.92$, is poor compared to $\chi_{r}^{2}=0.97$ for the MW dust model, so the MW fit would be strongly favored over the SMC model. Spectroscopy of the center of NGC 5866 (Smith et al. 2007) shows strong PAH emission features, consistent with the value $q_{\mathrm{PAH}}(\mathrm{MW}) \approx 2.0 \%$.

NGC 6822 is another case of a large discrepancy between $q_{\mathrm{PAH}}=0.7 \%$ obtained from MW models and the value $q_{\mathrm{PAH}}=$ $0.1 \%$ obtained when fitting is limited to LMC+SMC dust mod-
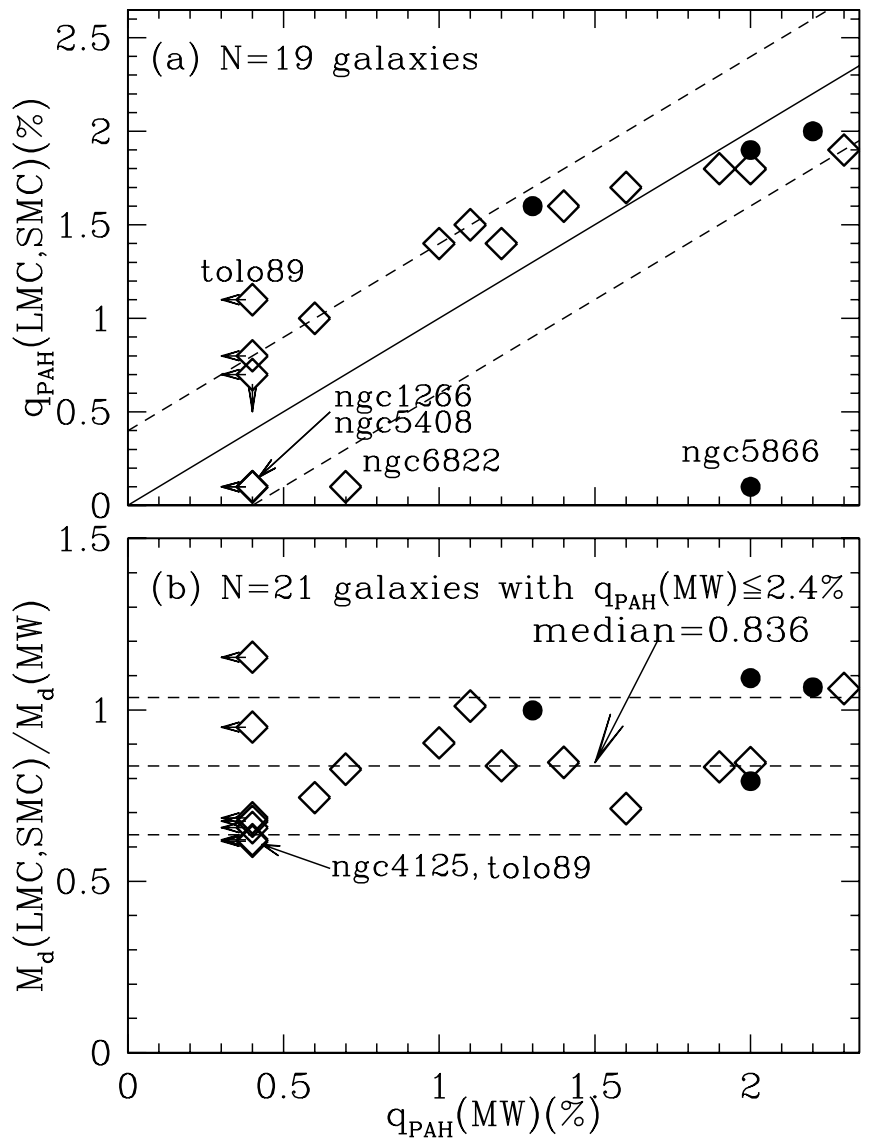

FIG. 5.- (a) $q_{\mathrm{PAH}}$ determined by fitting LMC or SMC models vs. $q_{\mathrm{PAH}}$ determined by fitting MW models, for galaxies with $q_{\mathrm{PAH}}(\mathrm{MW}) \leq 2.4 \%$. The estimates of $q_{\mathrm{PAH}}$ are in good agreement: $16 / 19$ galaxies have $\left|q_{\mathrm{PAH}}(\mathrm{MW})-q_{\mathrm{PAH}}(x \mathrm{MC})\right| \leq$ $0.4 \%$ (region bounded by dashed lines). (b) Ratio of dust masses for best-fit $\mathrm{LMC}+\mathrm{SMC}$ model and best-fit MW dust model, for galaxies with $q_{\mathrm{PAH}}(\mathrm{MW}) \leq$ $2.4 \%$. The dust masses are in good agreement: the median ratio is 0.836 , and $15 / 21$ galaxies are within \pm 0.20 of the median. [See the electronic edition of the Journal for a color version of this figure.]

els. Once again, the MW dust model produces a significantly better fit $\left(\chi_{r}^{2}=1.13\right)$ than the SMC model $\left(\chi_{r}^{2}=2.04\right)$.

For Tol $89(=\mathrm{NGC} 5398)$, on the other hand, $q_{\mathrm{PAH}} \leq 0.4 \%$ is obtained using the MW dust models, but the best-fit LMC+SMC model is an LMC dust model with $q_{\mathrm{PAH}}=1.1 \%$. In this case the fitting is confused by the apparent lack of dust emission in IRAC band 3, despite a strong dust excess in IRAC band 4. The MW fit has $\chi_{r}^{2}=2.50$, and the LMC fit has $\chi_{r}^{2}=2.47$, an indication that there is something wrong with either the data or the dust model.

Since 16 of the 19 galaxies have $q_{\mathrm{PAH}}(\mathrm{MW})-q_{\mathrm{PAH}}(x \mathrm{MC}) \mid \leq$ $0.4 \%$, we conclude that the estimates for $q_{\mathrm{PAH}}$ are generally not sensitive to the details of the trial dust models, and restricting the fitting to the MW dust models will give reasonable results for $q_{\mathrm{PAH}}$.

\subsubsection{Dust Mass}

Figure $5 b$ shows, for the 21 galaxies with $q_{\mathrm{PAH}}(\mathrm{MW})<2.4 \%$, the ratio of dust masses obtained using the two different dust models. The median ratio is 0.84 , with $15 / 21$ falling within the interval $M_{\text {dust }}(\mathrm{LMC}, \mathrm{SMC}) / M_{\text {dust }}(\mathrm{MW})=0.85 \pm 0.20$. It is clear that the inferred dust mass is relatively insensitive to the details of the model dust mixture used in the modeling.

Given that we obtain similar values for the dust mass $M_{\text {dust }}$ and the PAH abundance $q_{\text {PAH }}$ using either the MW or the LMC+ SMC dust models, we henceforth limit the modeling to the MW 


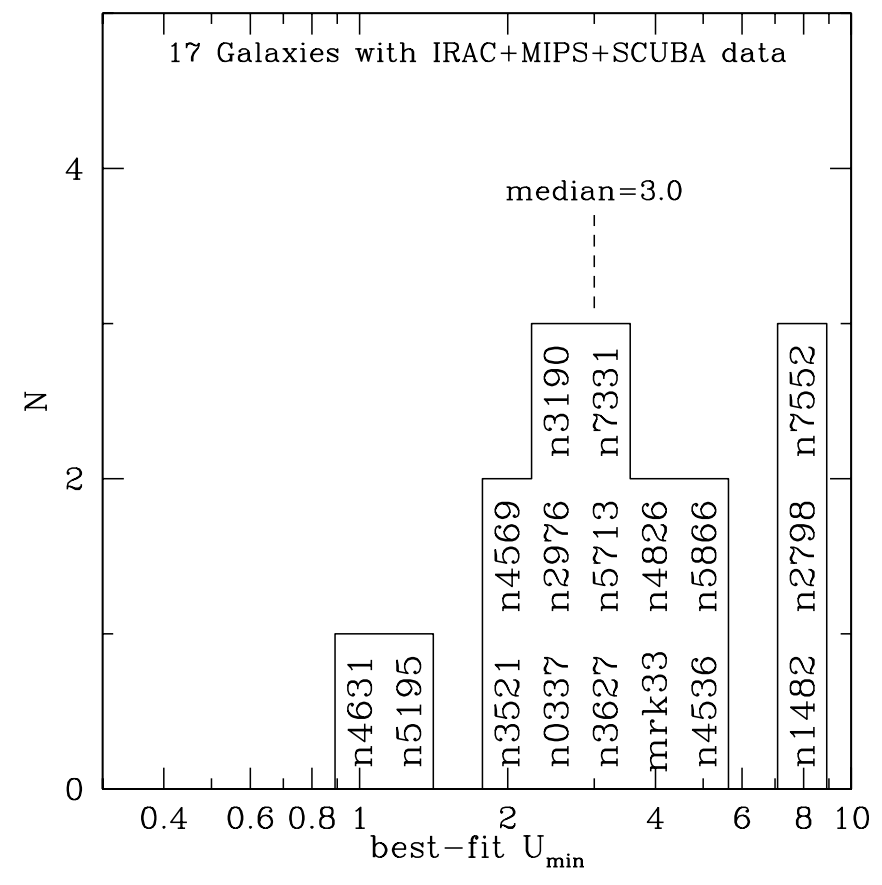

FIG. $6 a$

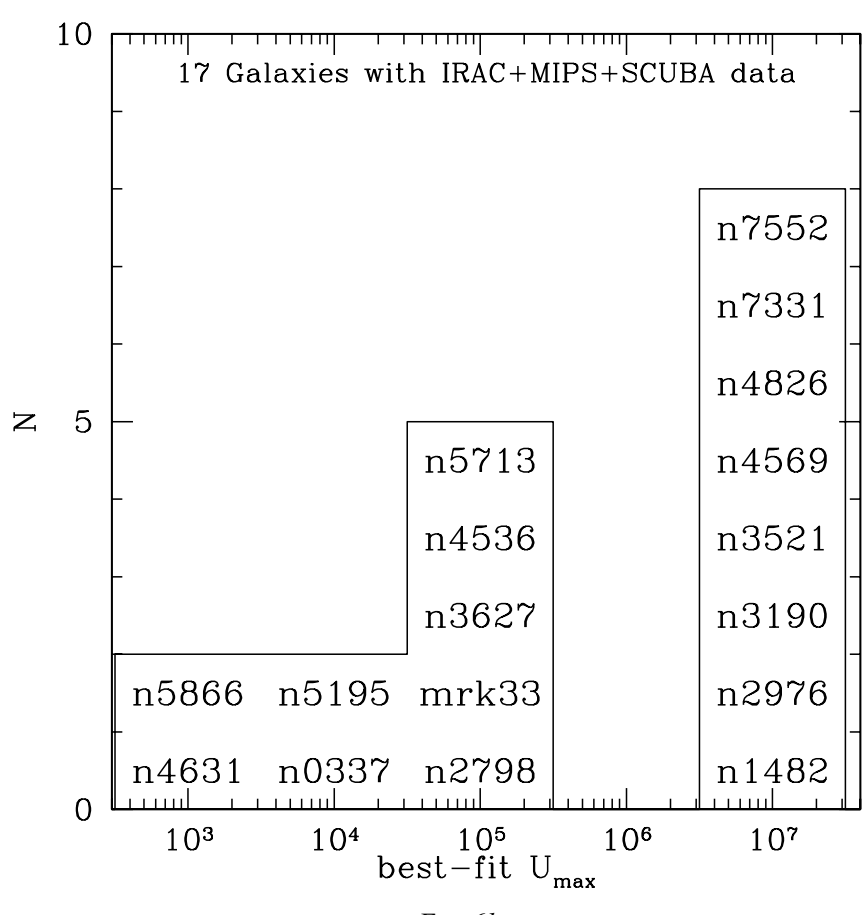

FIG. $6 b$

FIG. 6.- (a) Distribution of minimum starlight intensity scale factor $U_{\min }$. (b) Distribution of maximum starlight intensity scale factor $U_{\text {max }}$. The fits were limited to MW dust models.

dust models. This has the advantage of providing a set of models with $q_{\mathrm{PAH}}$ ranging from $0.4 \%$ to $4.6 \%$ (see Table 3 ), allowing systematic comparisons of the level of PAH emission among different galaxies. The resulting estimates for dust masses and starlight intensity distributions should be relatively robust, and even the PAH abundances appear to be reliable to within $\Delta q_{\mathrm{PAH}}=$ 0.004 . From this point on we limit the model fitting to the MW dust models.

\subsection{Radiation Field Parameters

$$
\text { 5.4.1. Lower Cutoff } U_{\min }
$$

Figure $6 a$ shows the distribution of the best-fit lower cutoff $U_{\min }$ for the SINGS-SCUBA galaxies. In no case did the best-fit model have $U_{\min }<1$, even though the model-fitting procedure considered values as small as 0.1 . We therefore conclude that, at least in the SINGS-SCUBA sample, only a very small fraction of the overall FIR emission is produced by cool grains within dark clouds (grains inside dark clouds, shielded from external starlight, will have $U \ll 1$ and will be cool, unless embedded star formation is present). The 160 and $850 \mu \mathrm{m}$ emission appears to be produced primarily by dust in diffuse regions with $U \gtrsim 1$. Cool grains in dark clouds could, in principle, contain a substantial fraction of the dust mass while contributing only a minor fraction of the total IR luminosity. However, we see below ( $\S 9.1)$ that the derived mass of dust heated by starlight with $U \gtrsim 0.7$ is generally close to the value expected based on the observed total (atomic and molecular) gas mass, indicating that the model-fitting procedure is not overlooking substantial amounts of cooler dust in dark regions.

\subsubsection{Upper Cutoff $U_{\max }$}

Figure $6 b$ also shows that the model-fitting procedure does not strongly favor any particular value of $U_{\max }$. We have experimented with fixing $U_{\max }$, and we find that fixing $U_{\max }=10^{5}$ results in only an increase in $\chi^{2}$ per galaxy of 0.47 , while the number of degrees of freedom per galaxy is increased by 1 . Fixing $U_{\max }=10^{6}$ works almost as well (the mean increase in $\chi^{2}$ per galaxy is 0.70 ), but fixing $U_{\max }$ at $10^{4}$ or $10^{7}$ results in larger increases in average $\chi^{2}$ per galaxy (in both cases $>1$ ) relative to the fits where $U_{\max }$ is allowed to vary.

Because of the insensitivity of the results to the precise value of $U_{\max }$, we simply set $U_{\max }=10^{6}$ and reduce the number of adjustable parameters from six to five: $\Omega_{\star}, M_{\text {dust }}, q_{\text {PAH }}, U_{\text {min }}$, and $\gamma$. Having $U_{\max }$ as large as $10^{6}$ is not surprising. The ultraviolet intensity in the Orion Bar PDR, for example, is $\sim 3 \times 10^{4}$ times stronger than the local starlight background (Marconi et al. 1998; Allers et al. 2005), and the dust within compact and ultracompact $\mathrm{H}$ II regions, as well as in the surrounding PDRs, is expected to be heated by radiation fields as large as $\sim 10^{6}$. We therefore expect the IR spectrum of a star-forming galaxy to have a noticeable contribution from dust heated by $U \approx 10^{6}$. From this point onward, we discuss only MW dust models restricted to $U_{\text {max }}=10^{6}$.

\subsubsection{Mean Intensity $\langle U\rangle$ and Fraction of Power from High-Intensity Regions}

Let $P_{0}$ be the power absorbed per unit dust mass in a radiation field $U=1$. For the intensity distribution given by equation (6) with $\alpha=2$, the dust luminosity $L_{\text {dust }}$ is obtained by integrating

$$
\begin{aligned}
d L_{\text {dust }}= & U P_{0} d M_{\text {dust }} \\
= & P_{0} M_{\text {dust }}\left[(1-\gamma) U \delta\left(U-U_{\text {min }}\right)\right. \\
& \left.+\frac{\gamma}{U_{\min }^{-1}-U_{\max }^{-1}} U^{-1}\right] d U .
\end{aligned}
$$

Thus, the total dust luminosity is

$$
L_{\text {dust }}=\langle U\rangle P_{0} M_{\text {dust }},
$$




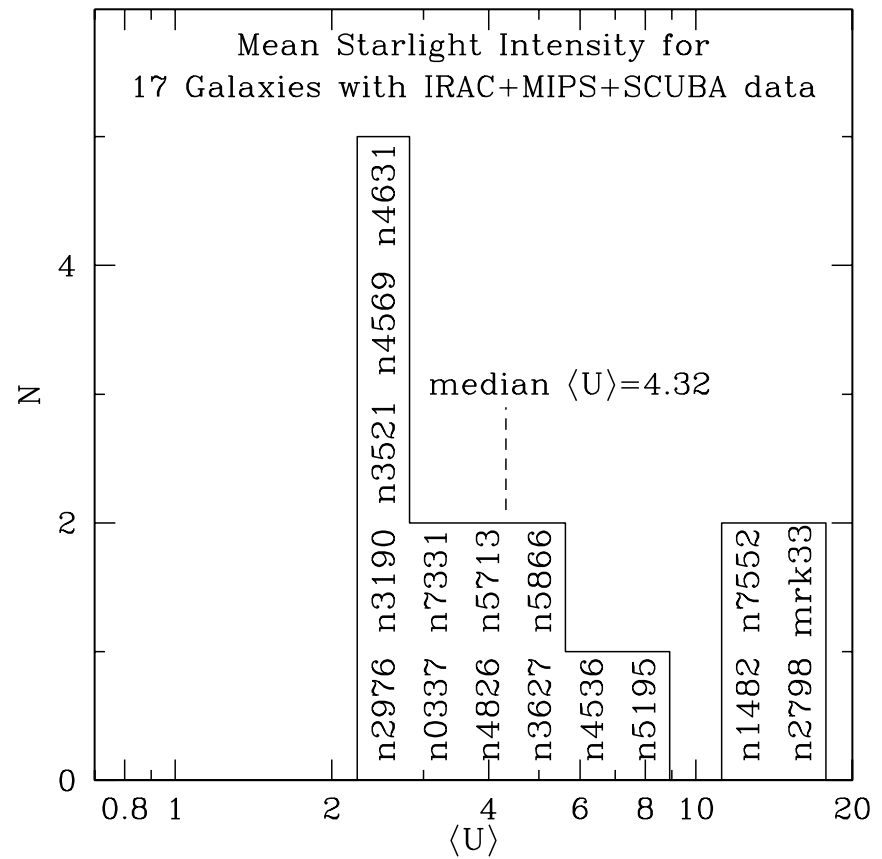

FIG. 7.-Distribution of $\langle U\rangle$ for 17 galaxies with SCUBA data (fits limited to MW dust with $U_{\max }=10^{6}$, with adjustable $\gamma$ and $U_{\min }$ ).

where the dust-weighted mean starlight intensity scale factor is

$$
\langle U\rangle=\left[(1-\gamma) U_{\min }+\frac{\gamma \ln \left(U_{\max } / U_{\min }\right)}{U_{\min }^{-1}-U_{\max }^{-1}}\right] .
$$

Figure 7 shows the distribution of $\langle U\rangle$ for the 17 SINGSSCUBA galaxies, fitted with MW dust models with $U_{\max }=10^{6}$. These galaxies are clearly not all alike: $\langle U\rangle$ varies by a factor of 6 over the sample, from $\langle U\rangle=2.6$ (for NGC 4569) to $\langle U\rangle=16$ (for Mrk 33), with median $\langle U\rangle=4.3$.

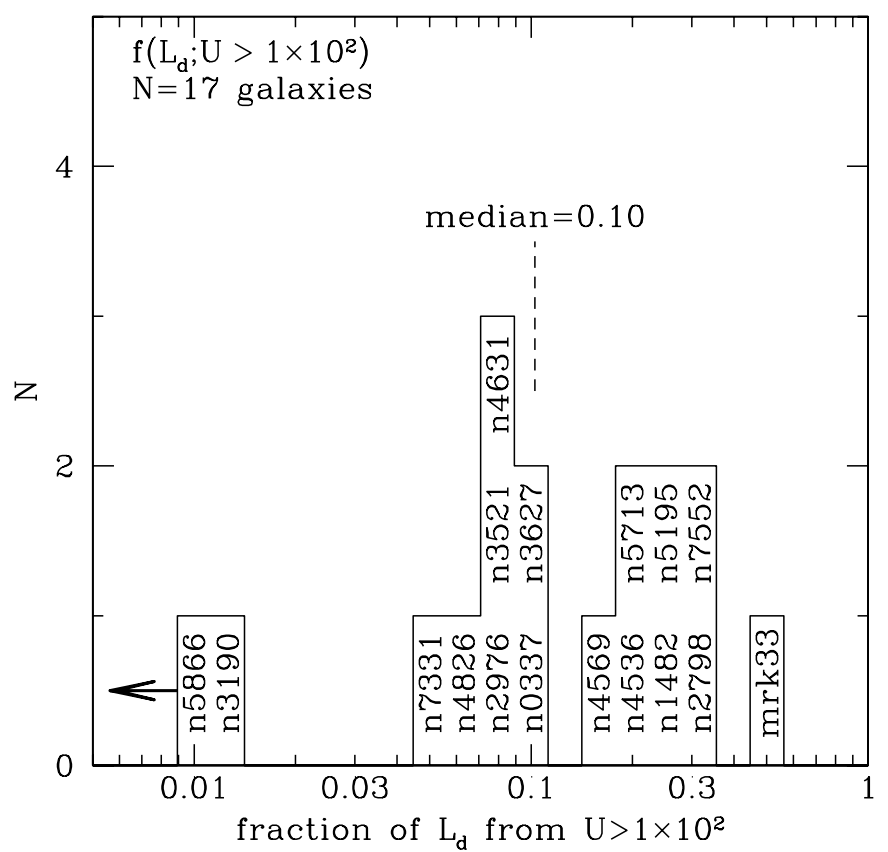

The fraction of the dust luminosity radiated from regions with $U>U_{c}$ (for $U_{\min }<U_{c}<U_{\max }$ ) is

$$
\begin{aligned}
& f\left(L_{\text {dust }} ; U>U_{c}\right) \\
& =\frac{\gamma \ln \left(U_{\max } / U_{c}\right)}{(1-\gamma)\left(1-U_{\min } / U_{\max }\right)+\gamma \ln \left(U_{\max } / U_{\min }\right)} .
\end{aligned}
$$

Figure 8 shows the distribution of $f\left(L_{\text {dust }} ; U>U_{c}\right)$ for $U_{c}=$ $10^{2}$ and $10^{3}$ for the 17 galaxies with SCUBA data. The median galaxy in the SINGS-SCUBA sample has $\sim 10 \%$ of the dust power originating in regions with $U>10^{2}$. In extreme cases, this fraction can be considerably larger, e.g., Mrk 33, with $f\left(L_{\text {dust }} ; U>\right.$ $\left.10^{2}\right) \approx 48 \%$, and $>30 \%$ for NGC 2798 and NGC 7552 . At the other extreme, the best-fit model for NGC 5866 (see $\S 5.6 .1$ ) has $\gamma=0$ and $f\left(L_{\text {dust }} ; U>10^{2}\right)=0$. Values of $U \gtrsim 10^{2}$ are expected to arise primarily in star-forming regions where dust is found near luminous stars. The values of $f\left(L_{\text {dust }} ; U>U_{c}\right)$ estimated from this fitting procedure are only approximate, but values of $f\left(L_{\text {dust }} ; U>10^{2}\right) \gtrsim 0.03$ should be indicative of significant star formation rates.

\subsection{PAH Abundances}

Observations with the Infrared Space Observatory (ISO) showed that normal star-forming galaxies routinely have strong 6-9 $\mu \mathrm{m}$ PAH emission (Dale et al. 2001). This is confirmed for the present sample of 17 SINGS-SCUBA galaxies. Figure 9 shows a histogram of $q_{\mathrm{PAH}}$ for these galaxies. All of the galaxies show PAH emission, with median $q_{\mathrm{PAH}}=3.5 \%$. None of these galaxies have $q_{\mathrm{PAH}}<1.3 \%$. The distribution of PAH abundances over a total of 61 SINGS galaxies is discussed in $\S 7.4$.

\subsection{Discussion of Selected Galaxies}

\subsubsection{Discrepant Cases: NGC 3521 and NGC 5866}

Most of the 17 galaxies in the SINGS-SCUBA sample have SEDs that are satisfactorily reproduced by the adopted dust model (see Fig. 3). If we consider only MW dust models, with fixed $U_{\max }=10^{6}$, the 17 galaxies have median $\chi_{r}^{2}=0.55 ; 16 / 17$

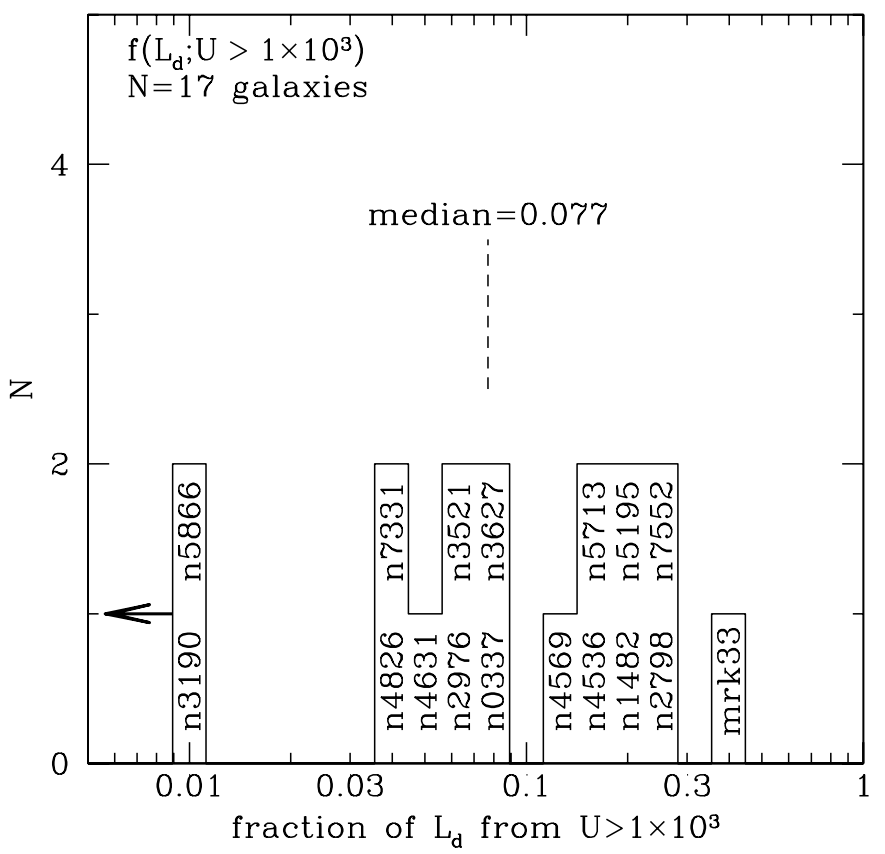

FIG. 8.-Distribution of the fraction of the dust luminosity radiated by dust exposed to starlight intensities $U>10^{2}$ and $U>10^{3}$. MW dust models are used for the fits, with fixed $U_{\max }=10^{6}$ and adjustable $\gamma$ and $U_{\min }$. Galaxies with $f \leq 0.01$ have been grouped in a bin at 0.01 . 


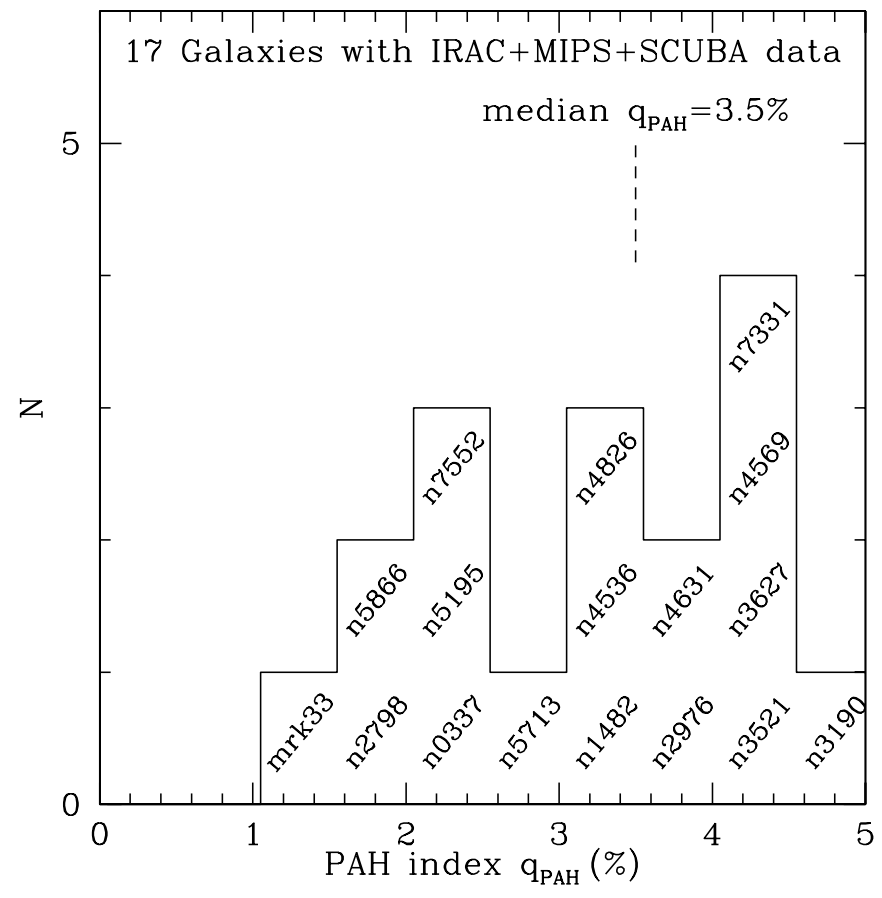

FIG. 9.- PAH index for 17 galaxies with SCUBA data, estimated by fitting MW dust models, with $U_{\max }$ allowed to vary.

of the galaxies have $\chi_{r}^{2}<1$. The SINGS-SCUBA galaxies with the worst fits (largest $\left.\chi_{r}^{2}\right)$ are NGC $3521\left(\chi_{r}^{2}=1.37\right)$ and NGC $5866\left(\chi_{r}^{2}=0.97\right)$. Here we consider why the model has difficulty fitting these two galaxies.

In the case of NGC 3521, the best-fit model underpredicts the $7.9 \mu \mathrm{m}$ emission, but the more significant discrepancies are at long wavelengths, where the model is high at $71 \mu \mathrm{m}$, low at $160 \mu \mathrm{m}$, and high at $850 \mu \mathrm{m}$. To illustrate the difficulty with reproducing these three fluxes, Figure 10 also shows a single-temperature component with a $\nu^{2}$ opacity with the temperature and power chosen to fit the 71 and $160 \mu \mathrm{m}$ data. Note that even this single-temperature

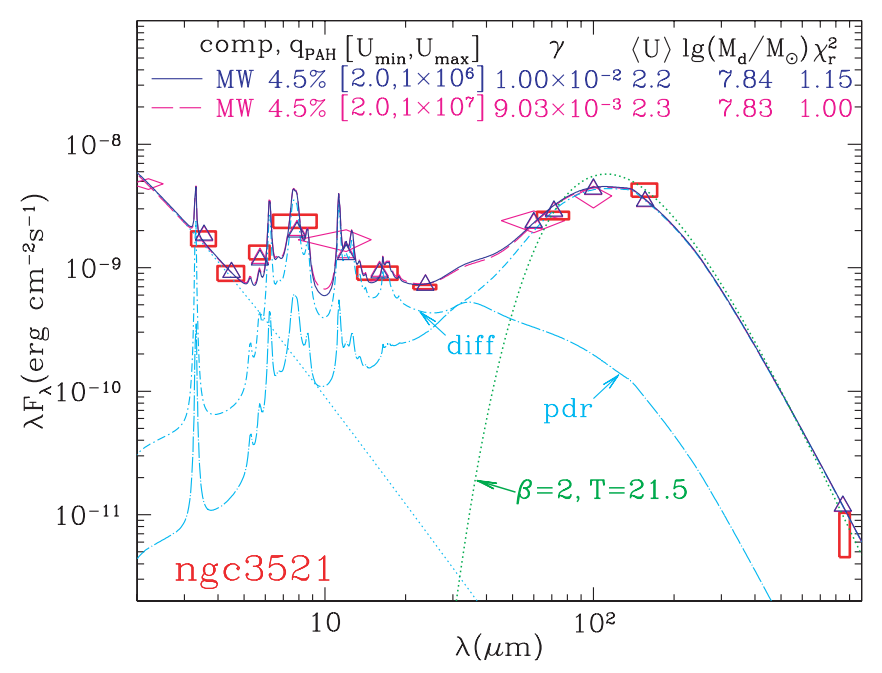

FIG. $10 a$ component is too "broad," i.e., it exceeds the $850 \mu \mathrm{m}$ datum. If a range of dust temperatures is present, it will broaden the spectrum and worsen the fit. Either there is a problem with some of the photometric data, or the dust in this galaxy has an opacity that is steeper than $\nu^{2}$.

The second-worst fit is for NGC 5866, an S0 galaxy with a substantial edge-on disk and the lowest value of $\left\langle\nu F_{\nu}\right\rangle_{24} /\left\langle\nu L_{\nu}\right\rangle_{71}$ among the SINGS-SCUBA galaxies (see Fig. 1). The best-fit model has $\gamma=0$ : all of the dust is exposed to starlight with intensity $U=U_{\min }=5$. The small value of $\gamma$ is presumably because the illumination of the dusty disk is dominated by the distributed old stellar population, with little contribution from star formation in the disk. This is consistent with nondetection of $\mathrm{H} \alpha$, with SFR $<0.1 M_{\odot} \mathrm{yr}^{-1}$ (Kennicutt et al. 2003) (although dust extinction may also be important). The nuclear spectrum of NGC 5866 (Smith et al. 2007) includes ionic emission lines (e.g., [Ne II], [Si II], [S III]), but they are relatively weak, consistent with only low levels of star formation; the low $[\mathrm{S}$ III $] /[\mathrm{Si}$ II $]$ flux ratio also suggests a deficiency of massive stars.

Roberts et al. (1991) estimated the dust mass to be $1.4 \times$ $10^{6} M_{\odot}$ (for our adopted distance of $12.5 \mathrm{Mpc}$ ). Here we estimate the dust mass to be $4.5 \times 10^{6} M_{\odot}$, larger by a factor of 3 . Our estimate for $M_{\text {dust }}$ results in $M_{\text {dust }} / M_{\mathrm{H}} \approx 0.005$, consistent with $A_{\mathrm{O}} \equiv \log _{10}(\mathrm{O} / \mathrm{H})+12=8.43 \pm 0.18$ (J. Moustakas et al. 2007, in preparation), $\sim 55 \%$ of solar $\left[\left(A_{\mathrm{O}}\right)_{\odot}=8.69\right.$ from Table 2].

Once again, the difficulty is in fitting the long-wavelength data. In this case, a single-temperature $T=24.5 \mathrm{~K}$ component with a $\nu^{2}$ opacity can fit the 71,160 , and $850 \mu \mathrm{m}$ photometry but exceeds the $450 \mu \mathrm{m}$ datum. Once again, a distribution of temperatures would exacerbate this problem, either indicating problems with the photometry or requiring an opacity substantially steeper than $\nu^{2}$ between 160 and $450 \mu \mathrm{m}$.

\subsubsection{Mrk 33}

The dwarf starburst galaxy Mrk 33 (see Fig. 11) has the highest value of $\left\langle\nu F_{\nu}\right\rangle_{24} /\left\langle\nu F_{\nu}\right\rangle_{71}$ among the SINGS-SCUBA galaxies (see Fig. 1). $\mathrm{H} \alpha$ observations of Mrk 33 indicate an SFR $\approx$ $1.5 M_{\odot} \mathrm{yr}^{-1}$, giving it the third-highest $\mathrm{SFR} / L_{\mathrm{opt}}$ ratio in the

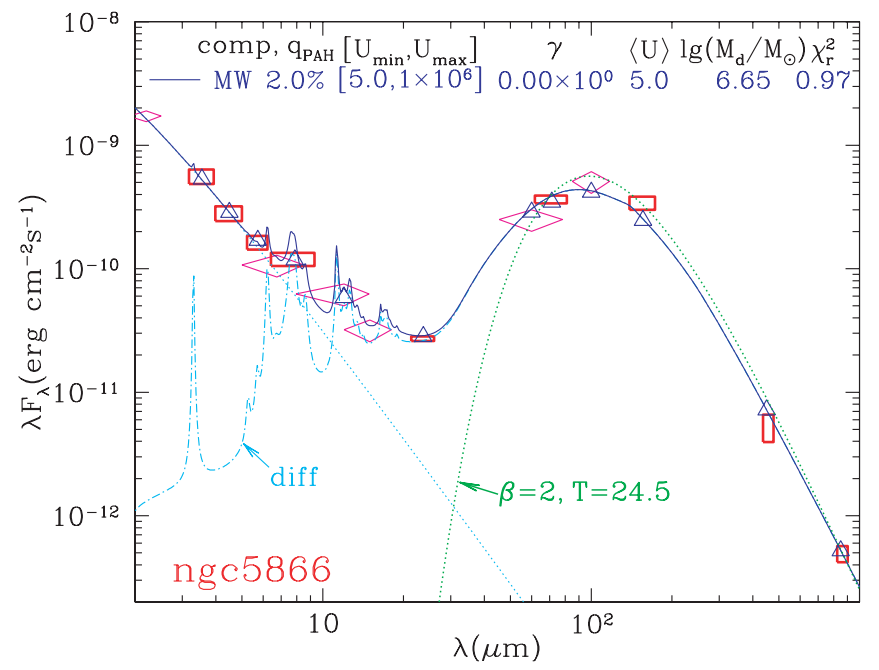

FIG. $10 b$

FIG. 10.- (a) SED of the SABbc galaxy NGC 3521, with $\chi_{r}^{2}=1.37$ for the best-fit MW dust model with $U_{\max }=10^{6}$. The dotted line shows emission from hypothetical $T=21.5 \mathrm{~K}$ dust with $\kappa_{\nu} \propto \nu^{2}$, showing that dust with $\kappa_{\nu} \propto \nu^{2}$ cannot reproduce the observed $160 \mu \mathrm{m}$ emission without exceeding either the $71 \mu \mathrm{m}$ flux (if $T>21.5 \mathrm{~K}$ ) or the $850 \mu \mathrm{m}$ flux (if $T<21.5 \mathrm{~K}$ ). Lines labeled "diff" and "pdr" show contributions of dust heated by $U=U_{\min }$ and $U>U_{\min }$, respectively, for the MW model with $U_{\max }=10^{6}$. (b) SED for the S0 galaxy NGC 5866. The best-fit MW dust model, with a single starlight intensity $U=5$, has $\chi_{r}^{2}=0.97$. The model has difficulty reproducing the sharpness of the peak in $\lambda F_{\lambda}$ near $100 \mu \mathrm{m}$ and overestimates the flux at $450 \mu \mathrm{m}$. 

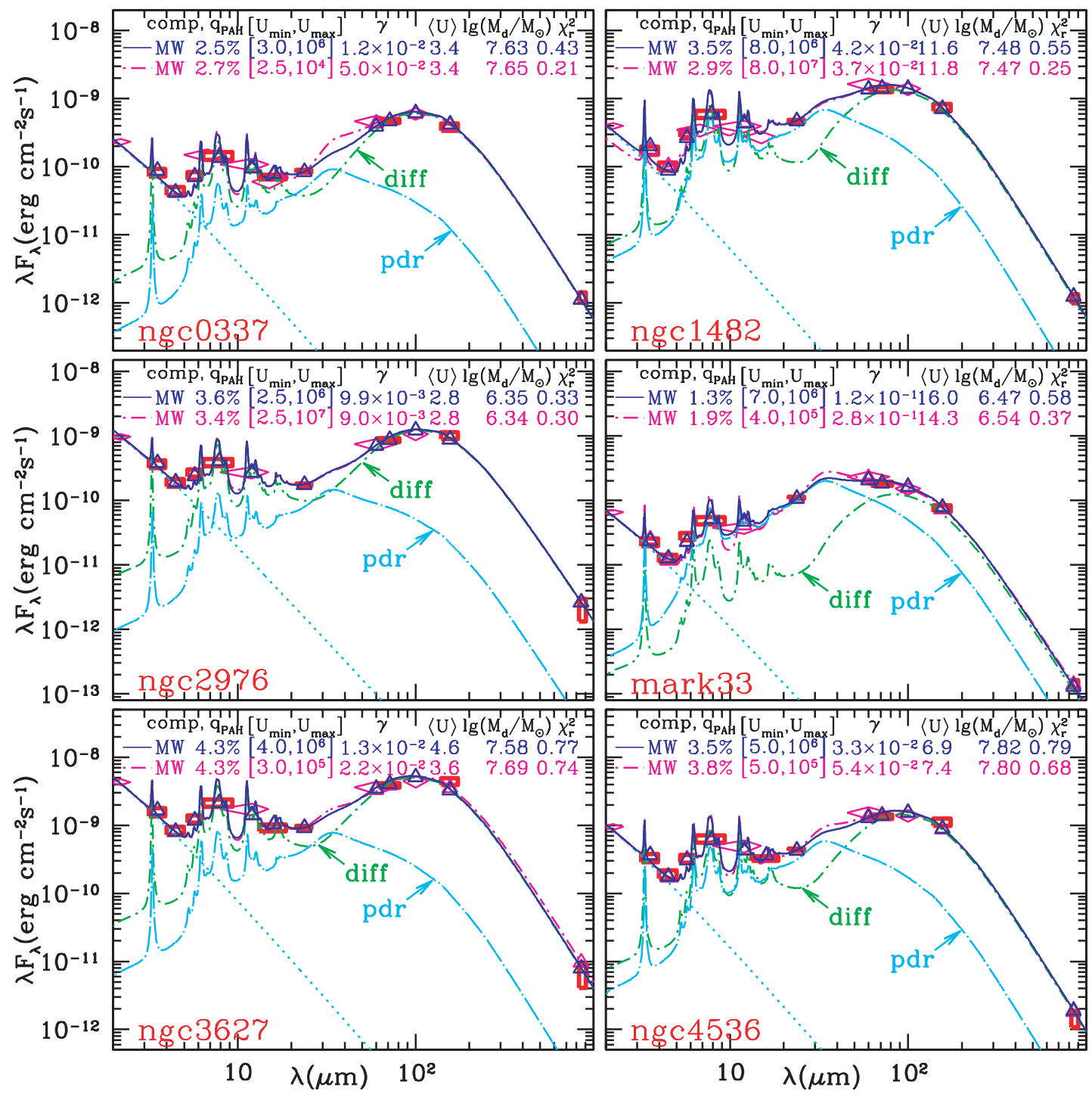

FIG. 11.-Observed fluxes and model emission spectra for galaxies with IRAC, MIPS, and IRAS data and at least one submillimeter flux measured by SCUBA. Some galaxies (e.g., NGC 3627) have also been measured in the IRS $16 \mu \mathrm{m}$ peak-up filter. Rectangles and diamonds show observed fluxes; vertical extent is $\pm 1 \sigma$. Lines show models fitted to IRAC, MIPS, SCUBA, and IRAS 12, 60, and $100 \mu \mathrm{m}$ data; triangles are models convolved with bandpass used for observations. Solid lines are models with MW dust and $U_{\max }=10^{6}$; dashed lines are used for other models that for some galaxies give smaller $\chi_{r}^{2}$ (see text). Dotted and dot-dashed lines show separate contributions of stars, dust with $U=U_{\min }$ (labeled "diff") and dust with $U>U_{\min }$ (labeled "pdr"). See Fig. 3 for NGC 2798 and NGC 3190 and Fig. 10 for NGC 3521 and NGC 5866

SINGS sample (Kennicutt et al. 2003). The best-fit $U_{\max }=10^{6}$ model has a very high mean starlight intensity $\langle U\rangle \approx 16$ and $f\left(L_{\text {dust }} ; U>10^{2}\right) \approx 48 \%$, the highest value in the sample.

\section{ESTIMATING DUST MASSES WITHOUT SUBMILLIMETER DATA}

Thus far we have been fitting dust models constrained by IRAC, MIPS, IRAS, and at least one submillimeter flux measured by SCUBA. Unfortunately, submillimeter observations are unavailable at this time for most of the SINGS galaxies. How well can dust masses be estimated in the absence of submillimeter constraints?

To address this question, we first repeat the dust modeling procedure used in $\S 5$ for the 17 SINGS-SCUBA galaxies, but with the SCUBA data omitted. The dust masses so obtained are shown in Figure 12, where they are plotted versus the dust masses estimated when SCUBA data are also used to constrain the models.
If the dust masses obtained with the SCUBA fluxes included are regarded as our "gold standard," it is clear that dropping the SCUBA data from the fitting procedure leads to substantial uncertainties in the model fitting. Five of the 17 galaxies have estimated dust masses that exceed the gold standard estimate by more than a factor of 1.5 (NGC 3521, NGC 3627, NGC 4536, NGC 4569, and NGC 7331). In five cases the dust mass estimate is lowered when the SCUBA data are not used, in one case by a factor of more than 1.5 (NGC 5195).

Figure 13 shows estimated dust-to-gas mass ratios for the 17 galaxies with SCUBA data for which the gas mass is known. Figure $13 a$ shows the dust/gas ratios estimated when the SCUBA fluxes are used in the fitting: the median dust/hydrogen mass ratio is 0.0053 for this sample, comparable to the value $\sim 0.007 \mathrm{ex}-$ pected for solar metallicity material with $\sim \frac{2}{3}$ of the $\mathrm{C}$ and most of the $\mathrm{Mg}, \mathrm{Si}$, and $\mathrm{Fe}$ incorporated into carbonaceous and silicate material (see Table 2). Figure $13 b$ shows the effect of fitting the SED without using the SCUBA data: for most of the galaxies, 

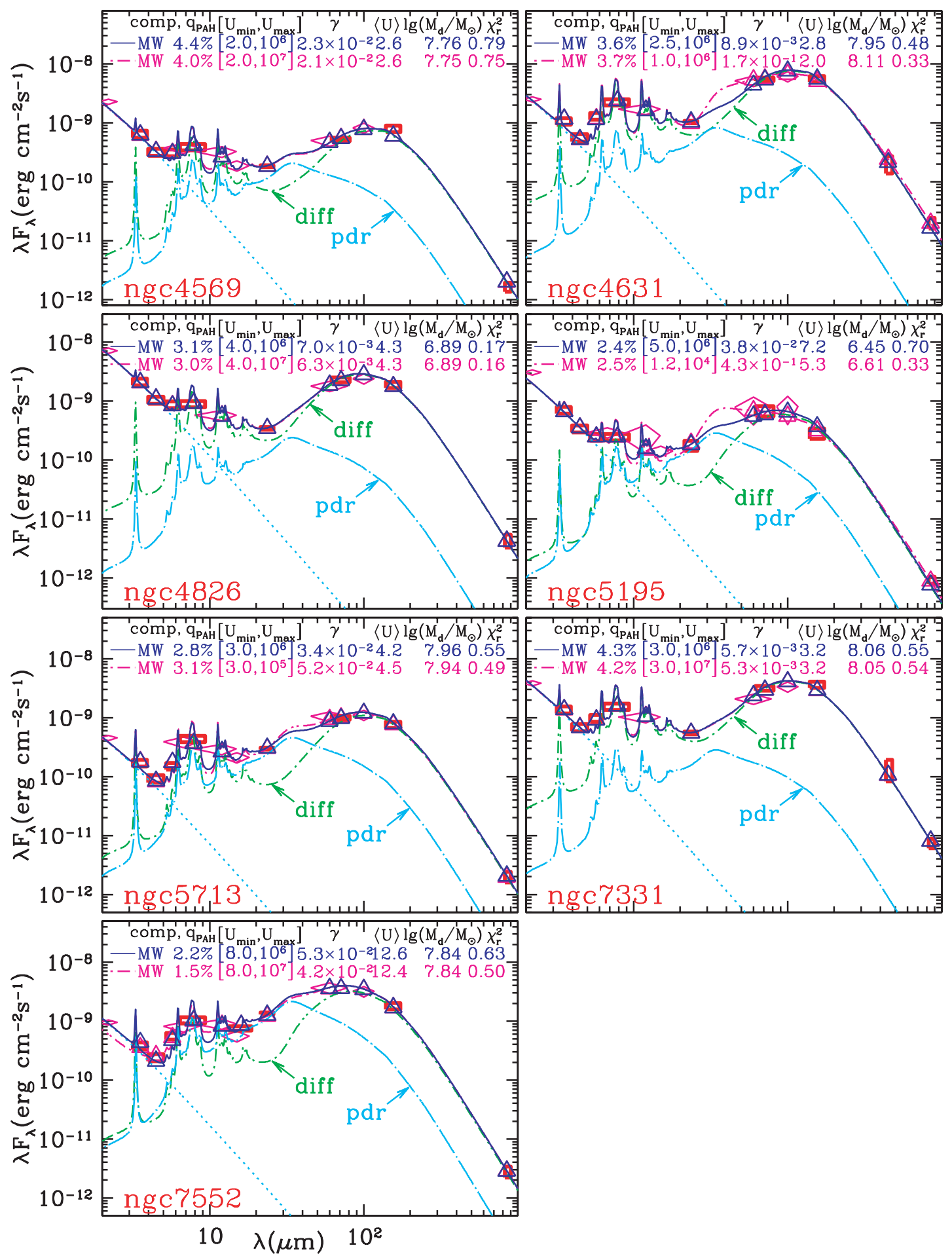

Fig. 11-Continued

the estimated dust/hydrogen ratio rises modestly, with a median value of only 0.0077 .

Because these models reproduce the SED fairly well and the IR power is mainly shortward of $200 \mu \mathrm{m}$, the total IR power is relatively unaffected when the SCUBA data are removed. What is changing is the estimated mean dust-weighted starlight intensity, with $\langle U\rangle$ often falling a factor of $\sim 1.5$ or more below the value obtained when the SCUBA data are employed. In the absence of submillimeter measurements to constrain the mass of cool dust, there is a risk that the model-fitting procedure may invoke a large mass of cool dust, heated by weak starlight. To prevent this, when submillimeter data are unavailable, we restrict the model fitting to use radiation fields with $U_{\text {min }} \geq 0.7$. This of course runs the risk of underestimating the dust mass if there are galaxies without submillimeter (e.g., SCUBA) observations that actually contain large amounts of cool dust heated by starlight with $U \lesssim 0.5$. However, we note that all the galaxies with SCUBA data had $U_{\min } \geq 1$ for the best-fit model (see Fig. $6 a$ ). 


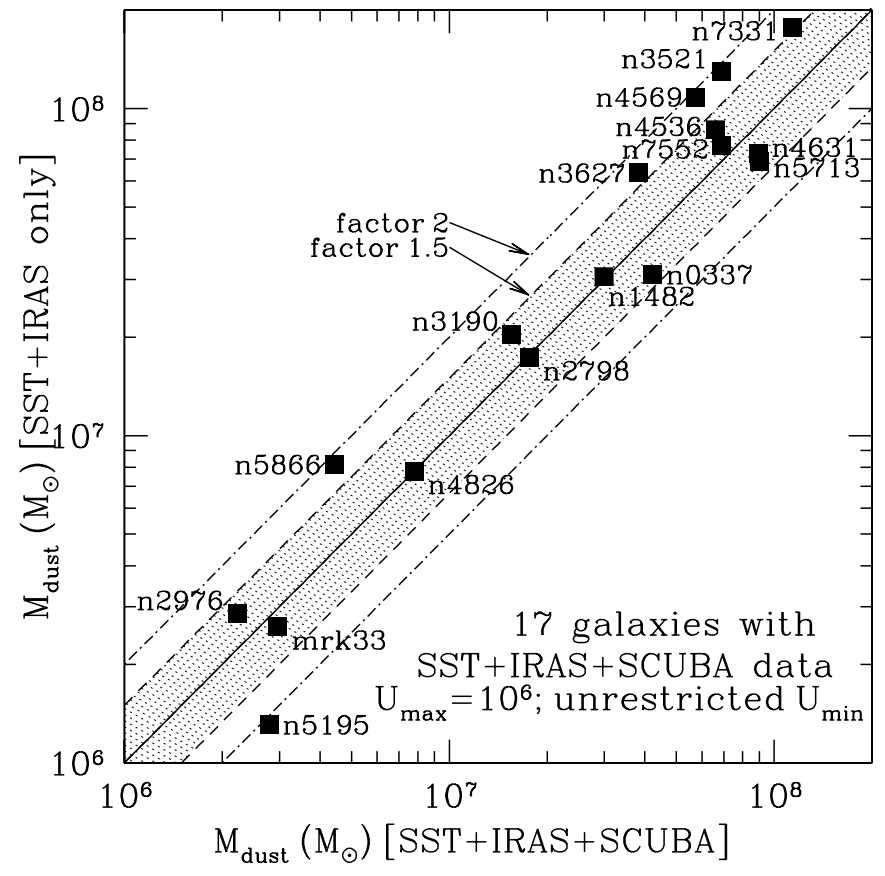

FIG. 12.-Dust mass $M_{\text {dust }}$ determined for 17 galaxies using IRAS and Spitzer data only, vs. the masses derived using IRAS, Spitzer, and SCUBA data combined. Data are fitted by MW dust models with $U_{\max }=10^{6}$ but no restriction on $U_{\min }$. Without SCUBA data, cool dust is not strongly constrained. Nevertheless, $11 / 17$ of the galaxies fall within a factor of 1.5 of the value obtained when SCUBA data are employed, and all 17 galaxies are within a factor of 2.2. [See the electronic edition of the Journal for a color version of this figure.]

Based on the above discussion, we adopt the following "restricted" fitting procedure when submillimeter data are unavailable:

1. Models limited to MW dust models only, with $0.4 \% \leq$ $q_{\mathrm{PAH}} \leq 4.6 \%$.

2. $U_{\max }=10^{6}$.

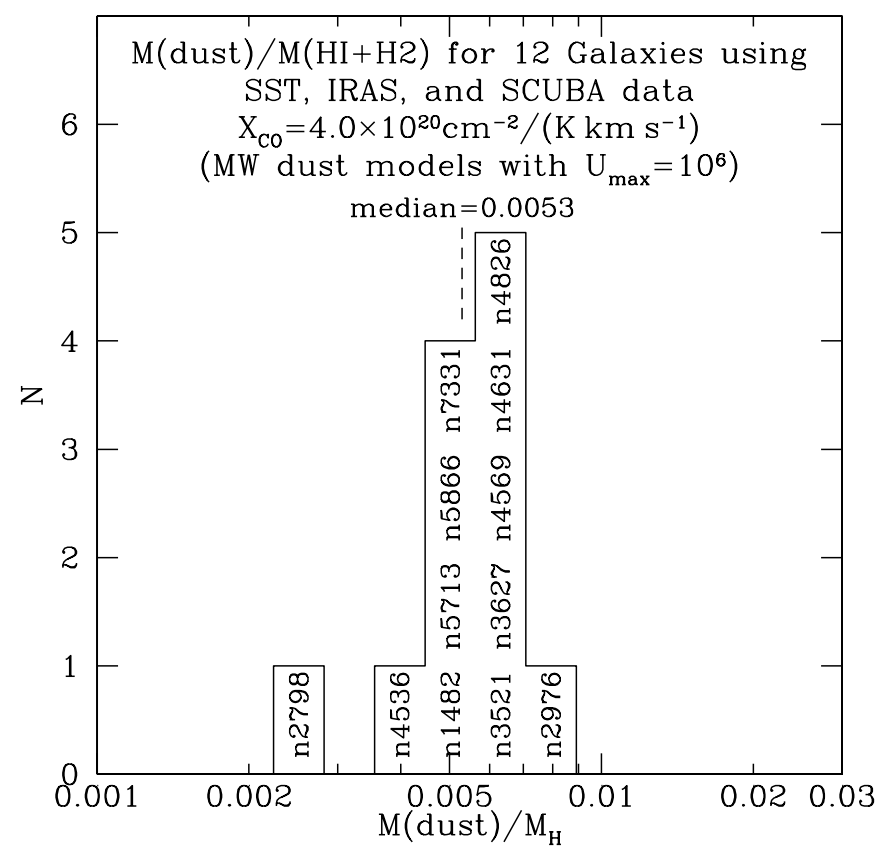

FIG. $13 a$
3. $\alpha=2$.

4. $0.7 \leq U_{\min } \leq 25$.

This restricted fitting procedure (with $U_{\min } \geq 0.7$ ) of course implies that the resulting fits will necessarily have $\langle U\rangle \geq 0.7$. This is generally consistent with what we observe for the sample of 17 galaxies with SCUBA data: as seen in Figure 7, these galaxies tend to have $\langle U\rangle \gtrsim 2$. However, the SINGS-SCUBA galaxy sample may be biased in favor of increased dust mass and associated star formation. If other galaxies have very low mean starlight intensities $\langle U\rangle$, the restricted fitting procedure adopted here would underestimate the dust mass (because it would overestimate $\langle U\rangle$ ). To study the dust in such galaxies, submillimeter observations are essential.

\section{DUST PROPERTIES FOR 65 SINGS GALAXIES}

\subsection{Dust-to-Gas Mass Ratio}

For 48 galaxies in the SINGS sample we have complete IRAC and MIPS data but no SCUBA fluxes. Dust masses have been estimated for these galaxies using the restricted fitting procedure described above. The SEDs for these galaxies, together with the best-fit models, are shown in Figure 14. The results for the dust mass $M_{\text {dust }}$ are given in Table 5.

For 20 of these galaxies both $\mathrm{H}$ I $21 \mathrm{~cm}$ and $\mathrm{CO} 2.6 \mathrm{~mm}$ fluxes have been detected (or there is a strong upper limit on the $\mathrm{CO}$ flux), and we are able to estimate the total gas mass. For another 24 galaxies $\mathrm{H}$ I $21 \mathrm{~cm}$ has been measured, but the CO $2.6 \mathrm{~mm}$ flux is unknown, so we have only a lower limit on the gas mass. For four galaxies (the E galaxies NGC 0855 and NGC 4125, and the S0 galaxies NGC 1266 and NGC 1316) neither H i nor CO has been detected.

Figure 15 shows the distribution of $M_{\text {dust }} / M_{\mathrm{H}}$ for the 20 galaxies lacking SCUBA data for which $M_{\mathrm{H}}$ is known. The distribution looks similar to the distribution in Figure 13b, except for two galaxies, IC 2574 and NGC 4236, with $M_{\text {dust }} / M_{\mathrm{H}} \approx 0.0005$. Note, however, that many of the sample galaxies do not appear on the histogram because $M_{\mathrm{H}}$ is not known.

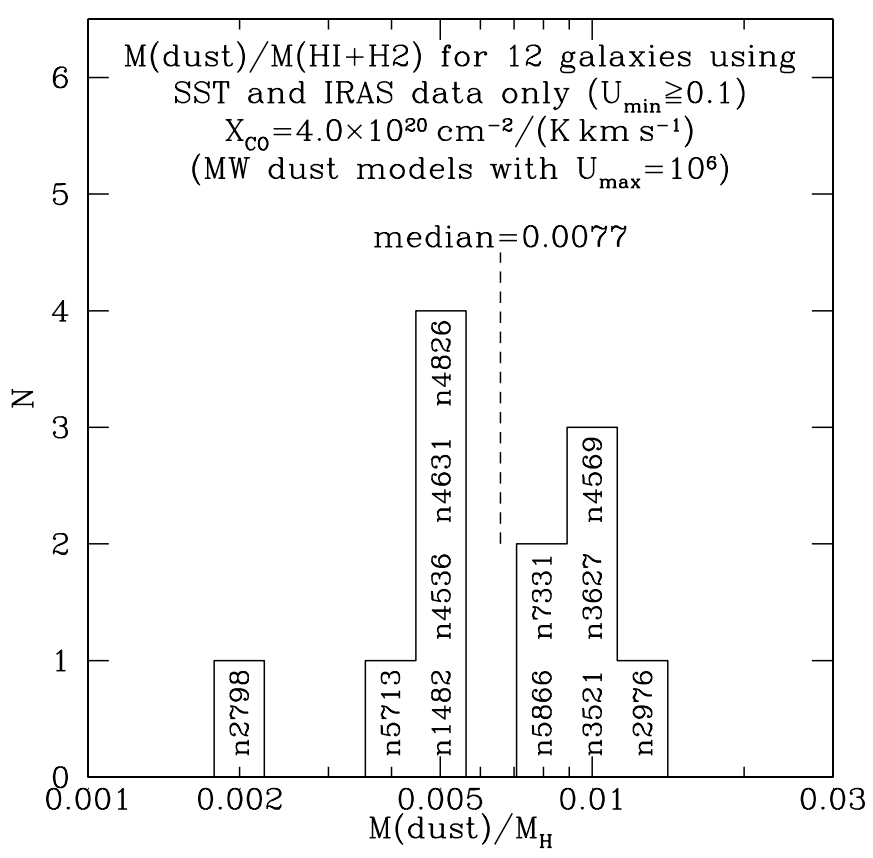

FIG. $13 b$

FIG. 13.- (a) Histogram of $M_{\mathrm{dust}} / M_{\mathrm{H}}$ obtained using IRAC+MIPS+SCUBA data for 12 galaxies for which $\mathrm{H}_{\mathrm{I}} 21 \mathrm{~cm}$ and $\mathrm{CO} 1-0$ fluxes have been measured. (b) Same as $(a)$, but estimated without using SCUBA data, with $U_{\max }=10^{6}$ but no restrictions on $U_{\min }$. 

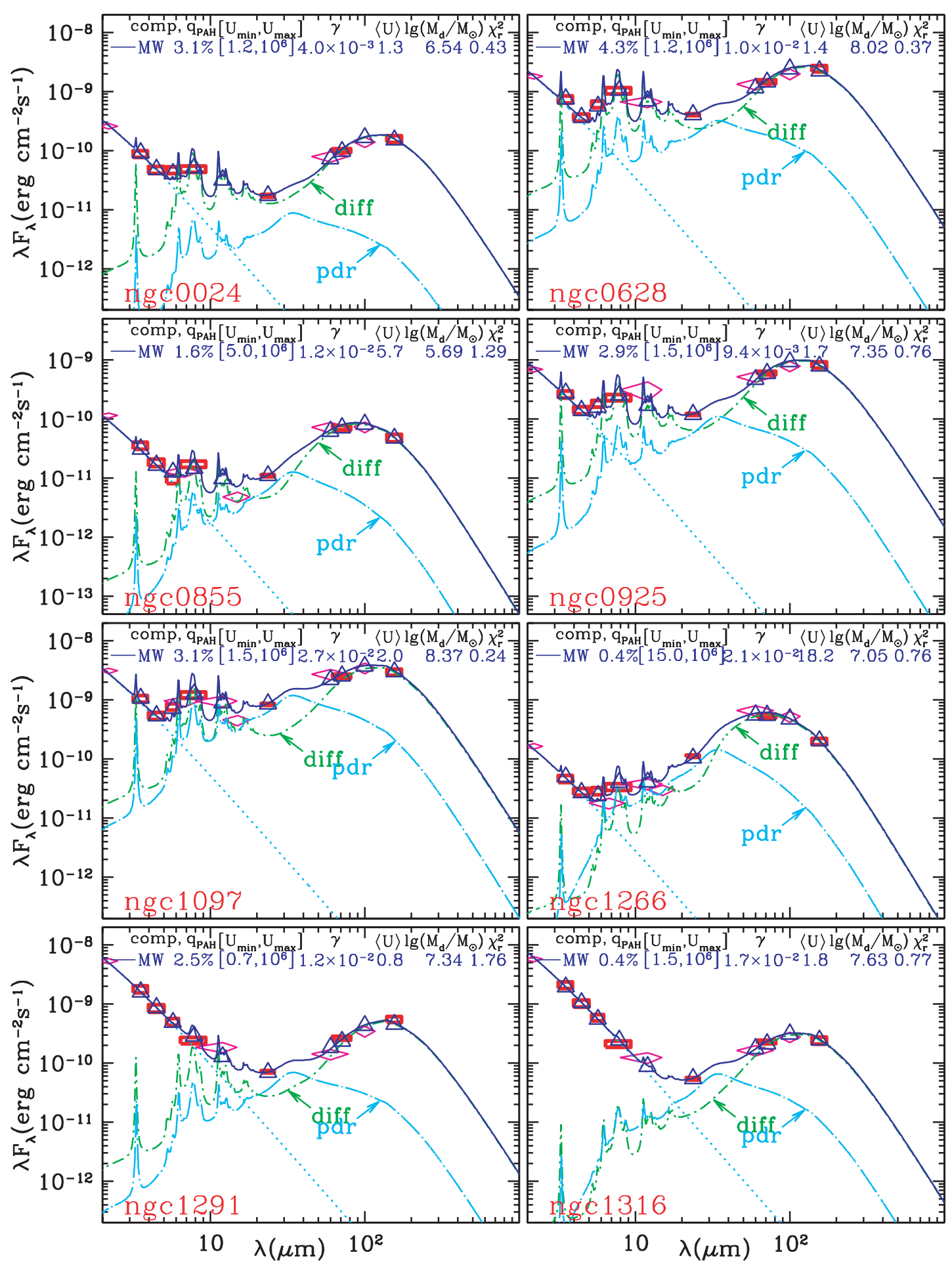

FIG. 14.- Same as Fig. 11, but for galaxies with only IRAC, MIPS, and IRAS data. [See the electronic edition of the Journal for additional panels of this figure.]

The dust-to-gas mass ratio reflects the enrichment of the gas by heavy elements $(\mathrm{C}, \mathrm{O}, \mathrm{Mg}, \mathrm{Si}, \mathrm{Fe})$ produced in stars; the production and growth of solid grains from these elements in supernovae, stellar winds, and the ISM; and destruction of grains by supernova blast waves and other violent events in the ISM.

If the same fraction of the condensable elements $(\mathrm{C}, \mathrm{Mg}, \mathrm{Si}$, $\mathrm{Fe}, \ldots$ ) is in grains as in the local $\mathrm{MW}$, then $M_{\text {dust }} / M_{\mathrm{H}}$ should conform to equation (13). However, it is possible that in some other galaxies, the balance between grain formation and destruction may be such that most interstellar $\mathrm{C}, \mathrm{Mg}, \mathrm{Si}$, and $\mathrm{Fe}$ is in the gas rather than in grains. We therefore expect equation (13) to define an upper envelope to the dust-to-gas mass ratio.

Models have been proposed to attempt to predict the dust-togas ratio in galaxies as they evolve in metallicity (Dwek 1998; Lisenfeld \& Ferrara 1998; Edmunds 2001; Hirashita et al. 2002). James et al. (2002) compared models to dust masses estimated from IR and submillimeter observations and argued that the fraction of the metals condensed into dust grains appeared to be the same in dwarf and giant galaxies, i.e., dust-to-gas mass ratios consistent with equation (13), for $8.1 \lesssim A_{\mathrm{O}} \lesssim 9.0$. 
TABLE 5

48 SingS Galaxies with IRAC and MIPS Global Fluxes, but No SCUBA Data

\begin{tabular}{|c|c|c|c|c|c|c|c|c|c|c|c|c|c|}
\hline Galaxy & $\begin{array}{c}\text { Morphological } \\
\text { Type }\end{array}$ & $\begin{array}{c}D^{\mathrm{a}} \\
(\mathrm{Mpc})\end{array}$ & $\begin{array}{c}\log M\left(\mathrm{H}_{\mathrm{I}}\right)^{\mathrm{a}} \\
\left(M_{\odot}\right)\end{array}$ & $\begin{array}{c}\log M\left(\mathrm{H}_{2}\right)^{\mathrm{a}, \mathrm{b}} \\
\left(M_{\odot}\right)\end{array}$ & $\begin{array}{c}\log M_{\text {dust }}{ }^{\mathrm{c}} \\
\left(M_{\odot}\right)\end{array}$ & $\begin{array}{c}\log L_{\text {dust }}{ }^{\mathrm{c}} \\
\left(L_{\odot}\right)\end{array}$ & $\begin{array}{l}q_{\mathrm{PAH}}{ }^{\mathrm{d}} \\
(\%)\end{array}$ & $\langle U\rangle^{\mathrm{e}}$ & $\begin{array}{l}\text { Dust } \\
\text { Type }\end{array}$ & $U_{\min }^{\mathrm{f}}$ & $\begin{array}{l}\gamma^{\mathrm{g}} \\
(\%)\end{array}$ & $\begin{array}{c}f\left(U>10^{2}\right)^{\mathrm{h}} \\
(\%)\end{array}$ & $\chi_{r}^{2 \mathrm{i}}$ \\
\hline NGC $0024 \ldots \ldots \ldots \ldots . . . .$. & $\mathrm{Sc}$ & 8.20 & 9.07 & $\ldots$ & 6.54 & 8.78 & 3.1 & 1.26 & MW & 1.2 & 0.40 & 3.5 & 0.43 \\
\hline NGC $0628 \ldots \ldots \ldots \ldots$ & $\mathrm{Sc}$ & 11.40 & $9.97^{\mathrm{j}}$ & $9.64^{\mathrm{k}}$ & 8.02 & 10.29 & 4.3 & 1.36 & MW & 1.2 & 1.04 & 8.4 & 0.37 \\
\hline NGC $0855 \ldots \ldots \ldots \ldots . . .$. & $\mathrm{E}$ & 9.60 & $\ldots$ & $\ldots$ & 5.69 & 8.58 & 1.6 & 5.70 & MW & 5.0 & 1.25 & 10.1 & 1.29 \\
\hline NGC $0925 \ldots \ldots \ldots \ldots . .$. & $\mathrm{Sd}$ & 10.10 & $9.75^{\mathrm{j}}$ & 9.18 & 7.35 & 9.71 & 2.9 & 1.67 & MW & 1.5 & 0.94 & 7.7 & 0.76 \\
\hline NGC $1097 \ldots \ldots \ldots \ldots . . .$. & $\mathrm{Sb}$ & 16.90 & 10.03 & $\ldots$ & 8.37 & 10.81 & 3.1 & 2.01 & MW & 1.5 & 2.72 & 18.7 & 0.24 \\
\hline NGC $1266 \ldots \ldots \ldots \ldots . . . .$. & So & 31.30 & $\ldots$ & $\ldots$ & 7.05 & 10.44 & 0.4 & 18.17 & MW & 15.0 & 2.09 & 15.9 & 0.76 \\
\hline NGC $1291 \ldots \ldots \ldots \ldots . . .$. & $\mathrm{S} 0 / \mathrm{a}$ & 9.70 & 9.19 & $\ldots$ & 7.34 & 9.38 & $2.5^{1}$ & 0.81 & MW & 0.7 & 1.17 & 9.4 & 1.76 \\
\hline NGC $1316 \ldots \ldots \ldots \ldots . . . .$. & S0 & 26.30 & $<8.87$ & $\ldots$ & 7.63 & 10.03 & $0.4^{1}$ & 1.82 & MW & 1.5 & 1.71 & 13.0 & 0.77 \\
\hline NGC $1512 \ldots \ldots \ldots \ldots$. & $\mathrm{Sa}$ & 10.40 & 9.77 & $\ldots$ & 7.21 & 9.45 & 3.8 & 1.28 & MW & 1.2 & 0.55 & 4.7 & 0.57 \\
\hline NGC $1566 \ldots \ldots \ldots \ldots . . .$. & Sbc & 18.00 & 10.01 & $\ldots$ & 8.25 & 10.62 & 4.0 & 1.72 & MW & 1.5 & 1.20 & 9.6 & 0.54 \\
\hline NGC $1705 \ldots \ldots \ldots \ldots$ & $\mathrm{Im}$ & $5.10^{\mathrm{m}}$ & $8.02^{\mathrm{j}, \mathrm{m}}$ & $\ldots$ & 4.86 & 7.89 & 0.6 & 7.72 & MW & 7.0 & 0.94 & 7.9 & 0.91 \\
\hline NGC $2403 \ldots \ldots \ldots . . . .$. & Scd & 3.50 & $9.48^{j}$ & 8.01 & 7.08 & 9.56 & 3.5 & 2.22 & MW & 2.0 & 0.90 & 7.5 & 0.62 \\
\hline Holmberg II............ & Im & $3.39^{\mathrm{n}}$ & $8.77^{\mathrm{j}}$ & $\ldots$ & 5.07 & 7.90 & 0.4 & 4.92 & MW & 4.0 & 2.01 & 15.1 & 4.13 \\
\hline DDO $053 \ldots \ldots \ldots \ldots . . . .$. & $\operatorname{Im}$ & 3.56 & $7.78^{j}$ & $\ldots$ & 4.00 & 7.08 & 1.1 & 8.95 & MW & 7.0 & 2.56 & 18.4 & 0.48 \\
\hline NGC $2841 \ldots \ldots \ldots \ldots . . . .$. & $\mathrm{Sb}$ & 9.80 & $9.62^{j}$ & 9.45 & 7.74 & 9.73 & 4.3 & 0.72 & MW & 0.7 & 0.21 & 1.8 & 0.66 \\
\hline NGC $2915 \ldots \ldots \ldots \ldots . . . .$. & $\operatorname{Im}$ & 2.70 & 8.25 & $\ldots$ & 4.14 & 7.33 & 1.4 & 11.22 & MW & 10.0 & 1.16 & 9.6 & 0.37 \\
\hline Holmberg I............. & $\mathrm{Im}$ & $3.84^{\mathrm{n}}$ & $8.15^{\mathrm{j}}$ & $\ldots$ & 4.83 & 7.16 & 1.2 & 1.58 & MW & 1.5 & 0.40 & 3.5 & 0.76 \\
\hline NGC $3049 \ldots \ldots \ldots \ldots . . . .$. & $\mathrm{Sab}$ & 19.60 & 9.10 & $\ldots$ & 6.74 & 9.58 & 2.9 & 5.13 & MW & 3.0 & 6.06 & 32.6 & 0.38 \\
\hline NGC $3031 \ldots \ldots \ldots \ldots . .$. & $\mathrm{Sab}$ & 3.50 & $9.53^{j}$ & $\ldots$ & 7.53 & 9.66 & 4.0 & 1.00 & MW & 1.0 & 0 & 0.0 & 0.33 \\
\hline NGC $3184 \ldots \ldots \ldots \ldots . . .$. & Scd & 8.60 & $9.26^{\mathrm{j}}$ & 9.11 & 7.70 & 9.76 & 4.5 & 0.86 & MW & 0.8 & 0.53 & 4.6 & 0.47 \\
\hline NGC $3198 \ldots \ldots \ldots \ldots . . . . .$. & $\mathrm{Sc}$ & 9.80 & $9.71^{\mathrm{j}}$ & $\ldots$ & 7.42 & 9.63 & 3.7 & 1.20 & MW & 1.0 & 1.58 & 12.1 & 0.48 \\
\hline IC $2574 \ldots \ldots \ldots \ldots \ldots$ & $\mathrm{Sm}$ & $4.02^{n}$ & $9.17^{\mathrm{j}}$ & $6.85^{\circ}$ & 5.86 & 8.36 & 0.4 & 2.30 & MW & 2.0 & 1.22 & 9.8 & 0.60 \\
\hline NGC $3265 \ldots \ldots \ldots \ldots . . .$. & $\mathrm{E}$ & 20.00 & 8.24 & $\ldots$ & 6.17 & 9.47 & 2.8 & 14.81 & MW & 10.0 & 4.58 & 28.5 & 0.14 \\
\hline NGC $3351 \ldots \ldots \ldots \ldots . . .$. & $\mathrm{Sb}$ & 9.30 & $9.01^{\mathrm{j}}$ & 8.98 & 7.46 & 9.89 & 3.2 & 1.95 & MW & 1.5 & 2.42 & 17.1 & 0.56 \\
\hline NGC $3621 \ldots \ldots \ldots \ldots$. & $\mathrm{Sd}$ & 6.20 & $9.79^{j}$ & $\ldots$ & 7.38 & 9.85 & 4.5 & 2.19 & MW & 2.0 & 0.78 & 6.5 & 0.68 \\
\hline NGC $3773 \ldots \ldots \ldots \ldots . . .$. & S0 & 12.90 & 7.99 & $\ldots$ & 5.90 & 8.86 & 2.3 & 6.82 & MW & 5.0 & 3.25 & 22.0 & 0.40 \\
\hline NGC $3938 \ldots \ldots \ldots \ldots . . . .$. & $\mathrm{Sc}$ & 12.20 & 9.57 & 9.61 & 7.69 & 9.94 & 4.6 & 1.30 & MW & 1.2 & 0.67 & 5.7 & 0.44 \\
\hline NGC $4125 \ldots \ldots \ldots \ldots . . . .$. & $\mathrm{E}$ & 21.40 & $\ldots$ & $\ldots$ & 6.38 & 9.07 & $0.4^{1}$ & 3.59 & MW & 3.0 & 1.69 & 13.0 & 0.38 \\
\hline NGC $4236 \ldots \ldots \ldots \ldots . . . .$. & $\mathrm{Sdm}$ & $4.45^{\mathrm{n}}$ & 9.44 & $<8.65$ & 6.15 & 8.67 & 1.0 & 2.41 & MW & 2.0 & 1.68 & 12.9 & 1.71 \\
\hline NGC $4254 \ldots \ldots \ldots \ldots . . .$. & $\mathrm{Sc}$ & 20.00 & 9.86 & 10.42 & 8.55 & 10.91 & 4.5 & 1.68 & MW & 1.5 & 0.96 & 7.9 & 0.72 \\
\hline NGC $4321 \ldots \ldots \ldots \ldots . .$. & Sbc & 20.00 & 9.67 & 10.32 & 8.57 & 10.82 & 4.2 & 1.33 & MW & 1.2 & 0.85 & 7.1 & 0.39 \\
\hline NGC $4450 \ldots \ldots \ldots \ldots . . .$. & $\mathrm{Sab}$ & 20.00 & 8.61 & 9.45 & 7.78 & 9.78 & 2.4 & 0.73 & MW & 0.7 & 0.36 & 3.1 & 0.30 \\
\hline NGC $4559 \ldots \ldots \ldots \ldots$ & Scd & 11.60 & 10.05 & $\ldots$ & 7.57 & 9.92 & 3.4 & 1.64 & MW & 1.5 & 0.73 & 6.1 & 0.69 \\
\hline NGC $4579 \ldots \ldots \ldots \ldots . . . . .$. & $\mathrm{Sb}$ & 20.00 & 8.91 & 9.75 & 8.18 & 10.25 & 3.5 & 0.86 & MW & 0.8 & 0.58 & 5.0 & 1.37 \\
\hline NGC $4594 \ldots \ldots \ldots \ldots . . . .$. & $\mathrm{Sa}$ & $9.25^{\mathrm{p}}$ & 8.43 & $8.65^{\mathrm{q}}$ & 7.56 & 9.55 & $3.7^{1}$ & 0.72 & MW & 0.7 & 0.24 & 2.1 & 1.00 \\
\hline NGC $4625 \ldots \ldots \ldots \ldots$. & $\mathrm{Sm}$ & 9.50 & 9.02 & $\ldots$ & 6.35 & 8.81 & 4.4 & 2.13 & MW & 2.0 & 0.53 & 4.6 & 0.22 \\
\hline NGC $4725 \ldots \ldots \ldots \ldots . .$. & $\mathrm{Sab}$ & 17.10 & 9.87 & 9.95 & 8.20 & 10.18 & 4.5 & 0.71 & MW & 0.7 & 0.16 & 1.4 & 0.57 \\
\hline NGC $4736 \ldots \ldots \ldots \ldots . . .$. & $\mathrm{Sab}$ & 5.30 & $8.71^{\mathrm{j}}$ & 9.05 & 7.11 & 9.89 & 4.1 & 4.37 & MW & 4.0 & 0.81 & 6.8 & 0.33 \\
\hline NGC $5033 \ldots \ldots \ldots \ldots . . . .$. & $\mathrm{Sc}$ & 13.30 & 9.97 & 9.66 & 7.95 & 10.28 & 4.1 & 1.61 & MW & 1.5 & 0.58 & 5.0 & 0.35 \\
\hline NGC $5055 \ldots \ldots \ldots \ldots . . .$. & Sbc & 8.20 & $9.78^{j}$ & 9.77 & 8.19 & 10.35 & 4.1 & 1.06 & MW & 1.0 & 0.48 & 4.1 & 0.31 \\
\hline NGC 5194............. & $\mathrm{Sbc}$ & 8.20 & $9.43^{\mathrm{j}}$ & 10.03 & 8.26 & 10.61 & 4.5 & 1.66 & MW & 1.5 & 0.84 & 7.0 & 0.40 \\
\hline Tol $89 \ldots \ldots \ldots \ldots \ldots \ldots$ & $\mathrm{Sdm}$ & 15.00 & 9.11 & $\ldots$ & 6.46 & 9.15 & 0.4 & 3.55 & MW & 2.0 & 6.41 & 33.2 & 2.50 \\
\hline NGC $5408 \ldots \ldots \ldots \ldots . . . .$. & Im & $4.81^{\mathrm{r}}$ & 8.51 & $\ldots$ & 4.67 & 8.28 & 0.4 & 29.45 & MW & 20.0 & 4.81 & 30.1 & 2.12 \\
\hline NGC $5474 \ldots \ldots \ldots \ldots$ & Scd & 6.90 & 9.10 & $\cdots$ & 6.39 & 8.74 & 1.9 & 1.63 & MW & 1.5 & 0.71 & 6.0 & 0.46 \\
\hline IC $4710 \ldots \ldots \ldots \ldots \ldots . . . . .$. & $\mathrm{Sm}$ & 8.50 & 8.51 & $\ldots$ & 5.71 & 8.60 & 2.0 & 5.73 & MW & 5.0 & 1.30 & 10.4 & 0.49 \\
\hline NGC $6822 \ldots \ldots \ldots \ldots . . .$. & $\mathrm{Im}$ & $0.49^{\mathrm{s}}$ & 8.13 & $\ldots$ & 5.04 & 7.62 & 0.7 & 2.77 & MW & 2.5 & 0.89 & 7.4 & 1.13 \\
\hline NGC $6946 \ldots \ldots \ldots \ldots . . .$. & Scd & 5.50 & $9.37^{t}$ & 9.77 & 7.74 & 10.35 & 4.3 & 3.03 & MW & 2.5 & 1.77 & 13.5 & 0.51 \\
\hline NGC $7793 \ldots \ldots \ldots \ldots . . .$. & $\mathrm{Sd}$ & 3.20 & $8.77^{\mathrm{j}}$ & $\ldots$ & 6.92 & 9.14 & 3.6 & 1.25 & MW & 1.2 & 0.31 & 2.7 & 0.85 \\
\hline
\end{tabular}

Note.-All models assume MW dust, with $U_{\max }=10^{6}$.

${ }^{a} D, 21 \mathrm{~cm}$ flux, and CO 1-0 flux from Kennicutt et al. (2003) unless otherwise noted.

b $M\left(\mathrm{H}_{2}\right)$ calculated using $X_{\mathrm{CO}}=4 \times 10^{20} \mathrm{~cm}^{-2}\left(\mathrm{~K} \mathrm{~km} \mathrm{~s}^{-1}\right)^{-1}$.

c Estimated from dust model.

${ }^{\mathrm{d}}$ Fraction of dust mass contributed by PAHs with $N_{\mathrm{C}}<10^{3} \mathrm{C}$ atoms.

e Dust-weighted mean starlight intensity scale factor.

${ }^{f}$ Lower cutoff for starlight intensity scale factor $U$.

g Fraction of dust mass exposed to $U>U_{\min }$.

h Fraction of dust luminosity from regions with $U>10^{2}$.

${ }^{i} \chi^{2} /\left(N_{b}-5\right)$, where $N_{b}$ is the number of bands used for fitting.

j $21 \mathrm{~cm}$ flux from $\mathrm{F}$. Walter (2005, private communication).

k CO 1-0 flux from Sheth et al. (2005).

${ }^{1}$ Parameter $q_{\text {PAH }}$ very uncertain; see $\S$ 7.4.

$\mathrm{m}$ Tosi et al. (2001).

${ }^{\mathrm{n}}$ Karachentsev et al. (2002a).

o CO 1-0 flux from Leroy et al. (2005).

p $D$ is the weighted mean of Ford et al. (1996) and Ajhar et al. (1997).

q CO 1-0 flux from Bajaja et al. (1991).

${ }^{\mathrm{r}}$ Karachentsev et al. (2002b).

s Cannon et al. (2005).

t $21 \mathrm{~cm}$ flux for radius $<6$ from Boulanger \& Viallefond (1992). 


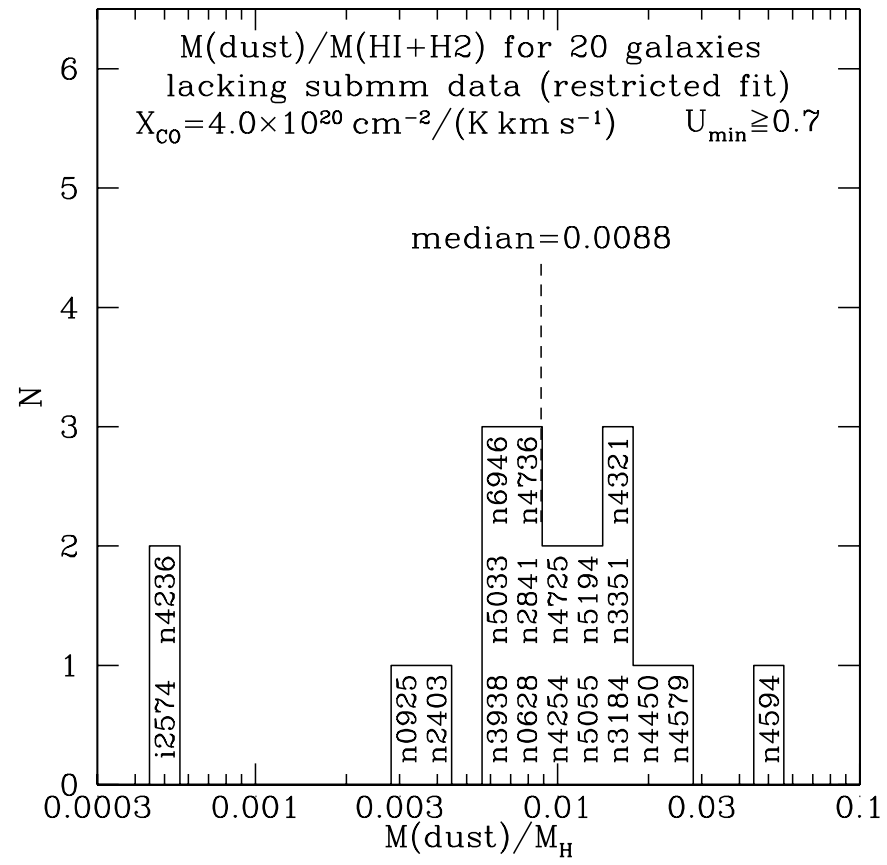

FIG. 15.- Dust-to-H mass ratio determined from IRAC+MIPS data only, using restricted fits with $U_{\min } \geq 0.7$ and taking $X_{\mathrm{CO}}=4 \times 10^{20} \mathrm{~cm}^{-2}\left(\mathrm{~K} \mathrm{~km} \mathrm{~s}^{-1}\right)^{-1}$.

Galliano et al. $(2003,2005)$ estimated the dust masses for four dwarf galaxies and found that NGC 1569 and He 2-10 had $M_{\text {dust }} / M_{\mathrm{H}}$ a factor of 4-7 and 2-10, respectively, below equation (13). Hunt et al. (2005) used IR and submillimeter observations to estimate dust masses for a sample of seven low-metallicity blue compact dwarfs. For SBS 0335-052, with metallicity $A_{\mathrm{O}}=$ $7.29 \pm 0.01$ (Izotov \& Thuan 1998), Hunt et al. (2005) found an extremely low dust-to-gas mass ratio $M_{\text {dust }} / M_{\mathrm{H}} \approx 10^{-7}$, far below the value $\sim 2 \times 10^{-4}$ given by equation (13). The results of Galliano et al. (2005) and Hunt et al. (2005) are clearly inconsistent with the conclusion of James et al. (2002) that dwarf galaxies have the same dust-to-metals ratio as the MW.

Figures $16 a$ and $16 b$ show how $M_{\text {dust }} / M_{\mathrm{H}}$ correlates with oxygen abundance for the SINGS galaxy sample, where we use the "characteristic" oxygen abundances ${ }^{19}$ from J. Moustakas et al. (2007, in preparation) based on only strong nebular lines. J. Moustakas et al. (2007, in preparation) show that different "strongline" methods (e.g., Kobulnicky \& Kewley 2004 vs. Pilyugin \& Thuan 2005) give differing estimates for $(\mathrm{O} / \mathrm{H})$, with the Pilyugin \& Thuan (2005) values tending to be smaller than the Kobulnicky $\&$ Kewley (2004) values by a factor of 4 , on average. Here we adopt abundances based on the Pilyugin \& Thuan (2005) method and refer the reader to J. Moustakas et al. (2007, in preparation) for discussion of the various systematic uncertainties. For the local MW we take the gas-phase $A_{\mathrm{O}}=8.59$ as determined by absorption-line studies of low-density sight lines (Cartledge et al. 2004). Figures $16 a$ and $16 b$ differ only in the assumed value of $X_{\mathrm{CO}}$, used to estimate the mass of $\mathrm{H}_{2}$. For both trial values of $X_{\mathrm{CO}}$, galaxies with $8.1 \lesssim A_{\mathrm{O}} \lesssim 8.5$ appear to conform to equation (13) to within a factor of $\sim 2$.

The two lowest metallicity galaxies in Figure 16, IC 2574 and NGC 4236, both fall well below equation (13), seemingly showing these systems to have only a small fraction of their

\footnotetext{
19 The characteristic abundance is intended to be representative of the luminosityweighted ISM material. For normal spirals, it is taken to be the metallicity at $0.4 R_{25}$ (J. Moustakas et al. 2007, in preparation). The quoted uncertainties in oxygen abundances do not include systematic uncertainties in the calibrations.
}

condensable elements in dust. However, we see below that this is not necessarily the case. The points labeled i2574* and n4236* show estimates for the dust/gas ratio for the regions in these galaxies where IR emission is observed (see $\S 7.2$ for further details). IC 2574 now falls right on equation (13). NGC 4236 is low by a factor of $\sim 2.5$, but the estimate for the mass of gas in the IR-emitting region is very uncertain ( $\operatorname{see} \S 7.2$ ).

The highest metallicity system in our sample is NGC 4594 (the Sombrero galaxy, M104). NGC 4594 also has the highest dustto-metals ratio; even for $X_{\mathrm{CO}}=4 \times 10^{20} \mathrm{~cm}^{-2}\left(\mathrm{~K} \mathrm{~km} \mathrm{~s}^{-1}\right)^{-1}$, $\left(M_{\text {dust }} / M_{\mathrm{H}}\right) /(\mathrm{O} / \mathrm{H})$ is a factor of $\sim 4$ above equation (13). Although this galaxy harbors a massive AGN, the dusty disk and ring are strongly detected at 24, 71, and $160 \mu \mathrm{m}$ (Bendo et al. 2006a). The dust-to-gas ratio estimated for the disk of this galaxy is quite high, $M_{\text {dust }} / M_{\mathrm{H}}=0.05$; the reason for this high value is not clear. Perhaps the dust properties in this system are significantly different from the MW dust properties that have been assumed in the modeling. Alternatively, perhaps the molecular mass in the dusty ring has been underestimated. The dusty ring has only been observed at a few positions in $\mathrm{CO}$; additional $\mathrm{CO}$ observations would be valuable. It is also possible that the ratio of $\mathrm{CO} \mathrm{lu}-$ minosity to molecular mass in this galaxy may differ from that in spiral galaxies where the so-called $\mathrm{X}$ factor has been calibrated. Unfortunately, the dust mass estimate for NGC 4594 is not yet constrained by submillimeter data: while NGC 4594 is strongly detected at $850 \mu \mathrm{m}$, the flux is dominated by the AGN, and the $850 \mu \mathrm{m}$ flux from the dusty disk has not yet been determined (Bendo et al. 2006a). We have seen above (Fig. 12) that dust mass estimates made with and without SCUBA data can differ by factors of $\sim 2$. Measurement of the submillimeter continuum emission from the dust ring in NGC 4594 would be of great value.

\subsection{Dust-to-Gas Mass Ratios in Low-Metallicity Galaxies}

There are many galaxies for which we have only lower limits on the gas mass because either $\mathrm{H}$ i or $\mathrm{CO}$ has not been observed. The resulting upper limits on $M_{\text {dust }} / M_{\mathrm{H}}$ are included in Figure 17, which now includes systems with $A_{\mathrm{O}}$ as low as $7.54 \pm 0.35$ (Holmberg I). For $A_{\mathrm{O}}>8.1$, upper limits on the global dust-togas ratio are generally consistent with equation (13), to within a factor of $\sim 2$. However, every galaxy in Figure 17 with $A_{\mathrm{O}}<8.1$ has a global dust-to-gas ratio that falls below equation (13), sometimes by a very large factor. IC 2574 is low by a factor of 5 , and four galaxies (Holmberg II, DDO 053, NGC 5408, NGC 2915) are factors of 8 or more below equation (13). The most extreme case is NGC 2915, below equation (13) by a factor of 40 . At face value, this might suggest that the balance between dust grain formation and destruction in low-metallicity galaxies such as NGC 2915 is much less favorable to grain formation and survival than in the MW. However, we argue here that (1) the dust-tometals ratio in the central regions of these galaxies is in fact normal (i.e., consistent with eq. [13]) and (2) the dust-to-metals ratio outside the central regions is not yet known.

Walter et al. (2007) compare the stellar, IR, and H I morphologies of the dwarf irregular ( Im) galaxies Holmberg II, Holmberg I, IC 2474, and DDO 053: in each of these galaxies, most of the $\mathrm{H}_{\mathrm{I}}$ is located outside the region where IR emission is detected. For example, only a fraction $f\left(\mathrm{H}_{\mathrm{I}}\right) \approx 0.19$ of the $\mathrm{H}$ i in IC 2574 lies within the regions where 70 and $160 \mu \mathrm{m}$ emission is seen. Similarly, the fraction of the $\mathrm{H}$ I within the contours where dust emission is detected is $f(\mathrm{H} \mathrm{I}) \approx 0.2,0.29$, and 0.33 for Holmberg II, Holmberg I, and DDO 053, respectively.

We have estimated the radii $\theta_{\mathrm{IR}}$ containing the bulk of the infrared emission for four additional low-metallicity dwarfs: 

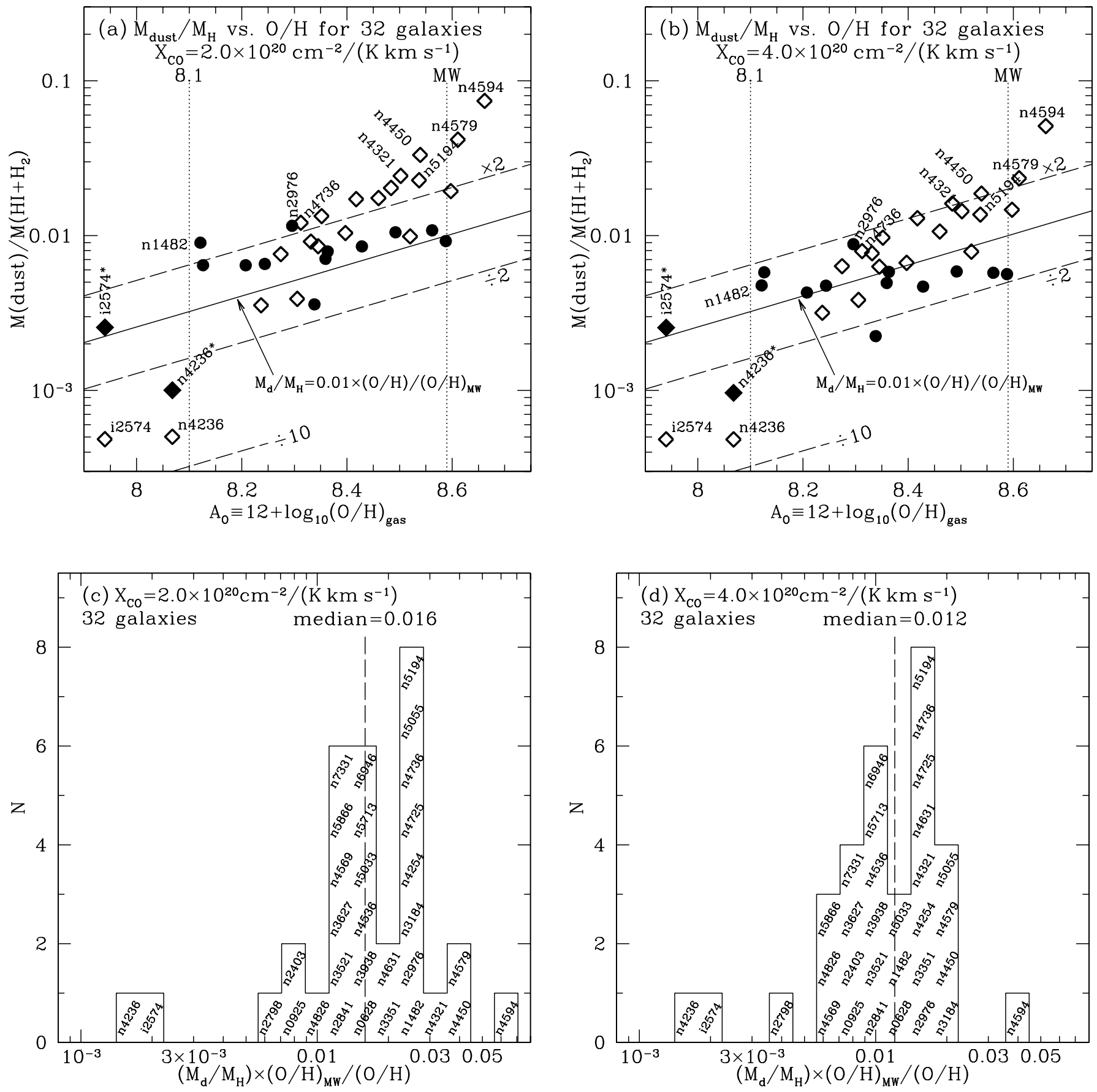

FIG. 16.- Ratio of $M_{\text {dust }}$ to $M_{\mathrm{H}}$ vs. oxygen abundance (see text) for galaxies with and without SCUBA fluxes (circles and diamonds, respectively), showing 32 galaxies

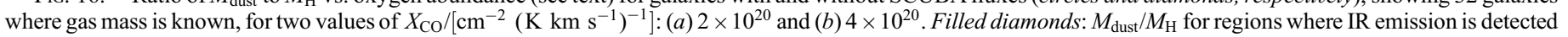

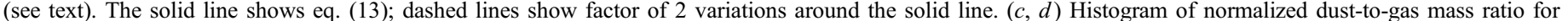
$X_{\mathrm{CO}} /\left[\mathrm{cm}^{-2}\left(\mathrm{~K} \mathrm{~km} \mathrm{~s}^{-1}\right)^{-1}\right]=2 \times 10^{20}$ and $4 \times 10^{20}$, respectively. [See the electronic edition of the Journal for a color version of panels a-b of this figure.]

NGC 1705 (Cannon et al. 2006a), NGC 2915, NGC 4236, and NGC 6822 (Cannon et al. 2006b). Our estimates for $\theta_{\mathrm{IR}}$ and for the fraction $f\left(\mathrm{H}\right.$ I) of the $\mathrm{H}_{\mathrm{I}}$ located at $\theta<\theta_{\mathrm{IR}}$ are given in Table 6 , with $f$ ranging from 0.028 (for NGC 2915) to 0.5 (for NGC 4236). The dust-to-gas mass ratios for the regions where IR emission is detected are shown in Figure 17. Six of the eight galaxies in Table 6 now have upper limits consistent with equation (13) to within a factor of 2, with only two (DDO 053 and NGC 4236) falling low by a factor of $\sim 2.5$. Given the uncertainties in estimation of both $M_{\text {dust }}$ and $f(\mathrm{H} \mathrm{I})$, it is entirely possible that $>50 \%$ of the interstellar $\mathrm{Mg}, \mathrm{Si}$, and $\mathrm{Fe}$ may be in solid grains, at least in systems with $A_{\mathrm{O}} \gtrsim 7.5$.
What can we say about the dust-to-metals ratio in the extended $\mathrm{H}$ I envelopes? Both the metal abundances and the dust masses in these regions are uncertain. The metallicities are estimated using $\mathrm{H}$ II regions and therefore apply only to regions with recent star formation. The outer $\mathrm{H}$ i envelopes lack bright $\mathrm{H}$ II regions, and therefore the metallicity there is unknown. It would not be surprising if it were substantially lower than the "characteristic" metallicities used in Figure 17. For the outer regions of some spiral galaxies, oxygen has been found to be underabundant, relative to the characteristic metallicity of the central regions, by factors of 4 or more (e.g., Ferguson et al. 1998; Tüllmann et al. 2003). 


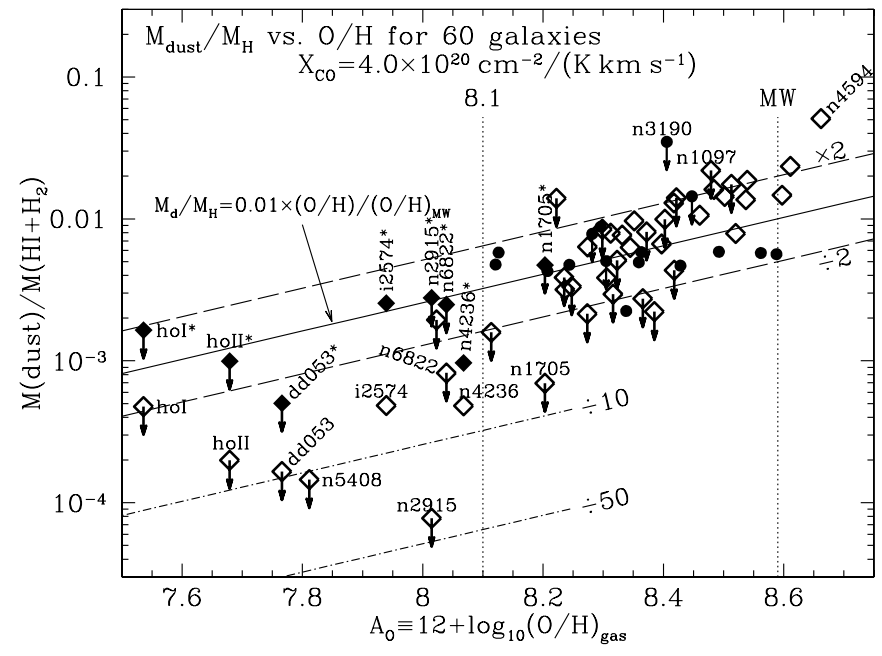

FIG. 17.-Galaxies with and without SCUBA fluxes (circles and diamonds, respectively), including 28 galaxies with only lower limits on the gas mass. The solid line shows eq. (13); dashed lines show factor of 2 variations around the solid line. Note that the upper bounds are all galaxies where $M_{\text {dust }}$ has been determined, but either $\mathrm{H}$ I or $\mathrm{CO}$ has not been observed. Filled diamonds show $M_{\text {dust }} / M_{\mathrm{H}}$ for regions where IR emission is detected (see $\S 7.2$ ). [See the electronic edition of the Journal for a color version of this figure.]

In the case of NGC 1705, UV absorption line studies show that the $\mathrm{H}$ I envelope has $A_{\mathrm{O}}=7.43 \pm 0.22$ (Heckman et al. 2001): the oxygen abundance in the $\mathrm{H}$ I envelope is a factor of 6 below the oxygen abundance determined from the $\mathrm{H}$ II regions (J. Moustakas et al. 2007, in preparation). For this oxygen abundance, $M_{\text {dust }} / M_{\mathrm{H}}$ for NGC 1705 is consistent with equation (13).

The dust content of the envelope is often also uncertain. The stellar densities in the outer regions of these galaxies are low, and the starlight will be dominated by light coming from the central regions. Table 6 lists $\left(\nu L_{\nu}\right)_{B}$, the total stellar luminosity in the $B$ band. For each of these galaxies we give the estimated half-mass radius $\theta_{0.5}$ for the $\mathrm{H}$ I, and we have estimated the starlight intensity scale factor $U$ at the $\mathrm{H}$ I half-mass radius,

$$
U_{0.5}=\frac{1}{\left(1.982 \times 10^{-13} \mathrm{ergs} \mathrm{cm}^{-3}\right)} \frac{\left(\nu L_{\nu}\right)_{B}}{4 \pi c\left(D \theta_{0.5}\right)^{2}},
$$

where $1.982 \times 10^{-13} \mathrm{ergs} \mathrm{cm}^{-3}$ is $\left(\nu u_{\nu}\right)_{B}$ for the local ISRF (Mathis et al. 1983). Equation (19) neglects internal extinction within the dwarf galaxy [illumination of the outer envelope will suffer the same attenuation as radiation escaping the galaxy, from which $\left(\nu L_{\nu}\right)_{B}$ was obtained]. Only one galaxy in Table 6, the dwarf starburst NGC 1705, has $U_{0.5}>0.2$.

The $a \gtrsim 0.03 \mu \mathrm{m}$ grains that normally dominate the $160 \mu \mathrm{m}$ emission are expected to have temperatures $T \approx 17 U^{1 / 6} \mathrm{~K}=$ $12(U / 0.1)^{1 / 6} \mathrm{~K}$, with peak $\nu p_{\nu}$ at $\lambda \approx h c / 6 k T \approx 180(0.2 / U)^{1 / 6} \mu \mathrm{m}$. Therefore, for the seven galaxies in Table 6 with $U_{0.5}<0.2$, even the MIPS $160 \mu \mathrm{m}$ band (with half-response points at 140 and $174 \mu \mathrm{m}$ ) is relatively insensitive to whatever dust may be present at and beyond $\theta_{0.5}$ except for a small fraction that may be close to local stellar sources. MIPS photometry for these galaxies is dominated by the dust in the luminous regions, and the SED fitting is therefore fitting the parameters for this dust. If there is dust present beyond the half-mass radius, submillimeter observations will be required to detect it in all of the galaxies in Table 6 except NGC 1705.

In the case of SBS 0335-052, where Hunt et al. (2005) report an extraordinarily low dust-to-gas mass ratio $M_{\text {dust }} / M_{\mathrm{H}}=1 \times 10^{-7}$, a factor of 5000 below equation (13), we note that this galaxy has not been detected at wavelengths between $100 \mu \mathrm{m}$ and $1 \mathrm{~cm}$. The dust models are poorly constrained, and it is possible that they may have significantly underestimated the mass of cool dust. In addition, much of the $\mathrm{H}$ I mass in SBS 0335-052 may be in an extended envelope, where the metallicity is unknown and where the dust temperatures would be very low. Deep submillimeter observations would be of value to better determine the dust content of SBS 0335-052.

\subsection{Dust-to-Gas Mass Ratio and Galaxy Type}

The dependence of the dust-to-gas mass ratio on galaxy type is explored in Figure 18. Spiral galaxies ( $\mathrm{Sab}, \mathrm{Sb}, \mathrm{Sbc}, \mathrm{Sc}, \mathrm{Scd}$, $\mathrm{Sd}$ ) routinely have $M_{\mathrm{dust}} / M_{\mathrm{H}} \approx 0.007$ to within a factor of 2 .

We have dust mass estimates for three elliptical galaxies (NGC 0855, NGC 3265, and NGC 4125) but lack information on the gas content of NGC 0855 and NGC 4125. Detection of $21 \mathrm{~cm}$ emission gives a lower bound on the gas mass in NGC 3265 . If the molecular and ionized gas mass in NGC 3265 is small compared to the $\mathrm{H}$ I mass, then this elliptical galaxy has a dustto-gas ratio consistent with $\sim$ solar metallicity.

The three S0 galaxies in our samples have dust-to-gas ratios that are consistent with the range seen for normal spirals: NGC 3773 has $M_{\text {dust }} / M_{\mathrm{H}}<0.01$, and NGC 1482 and NGC 5866 have $M_{\text {dust }} / M_{\mathrm{H}} \approx 0.005$.

TABLE 6

Parameters for Selected Dwarf Galaxies

\begin{tabular}{|c|c|c|c|c|c|c|c|c|}
\hline Galaxy & Morphological Type & $\begin{array}{c}D \\
(\mathrm{Mpc})\end{array}$ & $M_{B}$ & $\begin{array}{c}\left(\nu L_{\nu}\right)_{B} \\
\left(L_{\odot}\right)\end{array}$ & $\begin{array}{c}\theta_{\mathrm{IR}} \\
(\operatorname{arcmin})\end{array}$ & $\begin{array}{l}f\left(\mathrm{H} \mathrm{I}_{\mathrm{I}}\right. \\
\theta<\theta_{\mathrm{IR}}\end{array}$ & $\begin{array}{l}\theta_{0.5}(\mathrm{H} \mathrm{I}) \\
(\operatorname{arcmin})\end{array}$ & $U_{0.5}(\mathrm{H} \mathrm{I})$ \\
\hline DDO 053 & $\operatorname{Im}$ & 3.56 & -13.44 & $2.1 \times 10^{7}$ & $\ldots$ & $0.33^{\mathrm{a}}$ & 1 & 0.11 \\
\hline 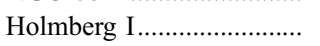 & Im & 3.84 & -15.12 & $1.0 \times 10^{8}$ & & $0.29^{\mathrm{c}}$ & 2 & 0.11 \\
\hline NGC $1705 \ldots \ldots \ldots \ldots \ldots \ldots \ldots$ & $\mathrm{Im}$ & 5.10 & -15.76 & $1.8 \times 10^{8}$ & $0.33^{\mathrm{d}}$ & 0.15 & $0.69^{\mathrm{e}}$ & 4.0 \\
\hline 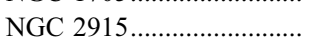 & $\operatorname{Im}$ & 2.70 & -16.41 & $3.3 \times 10^{8}$ & 0.5 & $0.028^{\mathrm{f}}$ & $4.75^{\mathrm{a}}$ & 0.13 \\
\hline 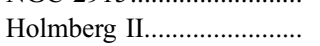 & $\operatorname{Im}$ & 3.39 & -16.69 & $4.2 \times 10^{8}$ & $\ldots$ & $0.20^{\mathrm{c}}$ & 4 & 0.15 \\
\hline
\end{tabular}

\footnotetext{
${ }^{\text {a }}$ Becker et al. (1988).

b Estimated from H I profile in Cannon et al. (2006b).

c Walter et al. (2007).

d Cannon et al. (2006a)

${ }^{\mathrm{e}}$ Meurer et al. (1998).

${ }^{f}$ Estimated from H I data in Meurer (1997).

$\mathrm{g}$ Estimated from Rots (1980) map, assuming FWHM $=10^{\prime}$ Gaussian beam.
} 


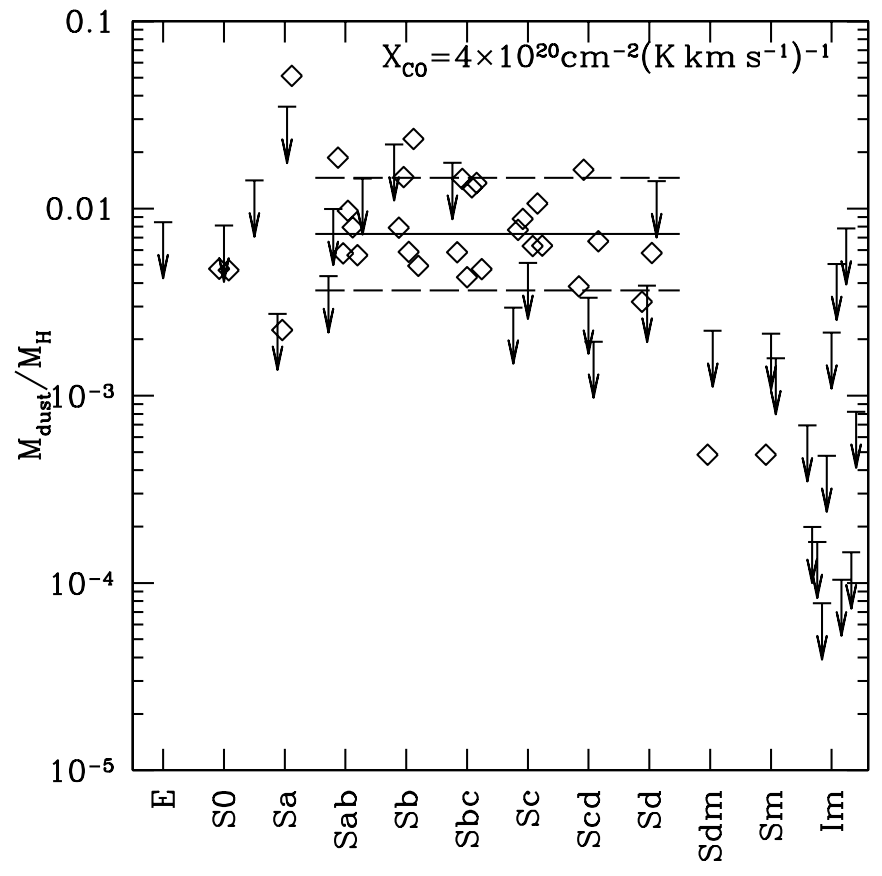

FIG. 18.-Dust-to-gas mass ratio as a function of Hubble type, assuming $X_{\mathrm{CO}}=4 \times 10^{20} \mathrm{~cm}^{-2}\left(\mathrm{~K} \mathrm{~km} \mathrm{~s}^{-1}\right)^{-1}$. The solid horizontal line corresponds to $M_{\text {dust }} / M_{\mathrm{H}}=0.0073$, and the dashed lines to factor of 2 variations around this. Note that the upper bounds are all cases where the dust mass has been estimated, but only lower bounds are available for the gas mass because $\mathrm{CO}$ has not yet been observed. [See the electronic edition of the Journal for a color version of this figure.]

\subsection{PAH Abundance}

The set of 65 SINGS galaxies includes galaxies with strong PAH emission detected in IRAC bands 3 and 4, as well as cases where there is little evidence of nonstellar emission in these bands. For some galaxies the dust emission (relative to starlight) is so weak that $q_{\text {PAH }}$ (the fraction of the total dust mass contributed by PAHs with $N_{\mathrm{C}}<10^{3}$ ) cannot be reliably determined from the broadband photometry. The DL07 models that are used here have

$$
\left\langle\nu F_{\nu}^{\mathrm{ns}}\right\rangle_{7.9 \mu \mathrm{m}} \approx 0.070\left(\frac{q_{\mathrm{PAH}}}{0.01}\right)\left(\frac{L_{\mathrm{dust}}}{4 \pi D^{2}}\right) .
$$

With the existing uncertainties in IRAC calibration and foreground/background subtraction, we do not attempt to estimate $q_{\text {PAH }}$ unless

$$
\left[\nu B_{\nu}\left(T_{\star}\right)\right]_{7.9 \mu \mathrm{m}} \Omega_{\star}<0.15\left(\frac{L_{\mathrm{dust}}}{4 \pi D^{2}}\right),
$$

so that the PAH power in the IRAC $7.9 \mu \mathrm{m}$ band would be $>50 \%$ of the stellar contribution if $q_{\mathrm{PAH}}=0.01$. This implies no estimate of $q_{\mathrm{PAH}}$ for four galaxies: NGC 1291, NGC 1316, NGC 4125, and NGC 4594, all galaxies with relatively large bulge/ disk ratios.

Figure 19 shows the distribution of $q_{\mathrm{PAH}}$ for the remaining 61 galaxies for which we have both IRAC and MIPS data. The distribution is broad: $7 / 61$ of the galaxies have $q_{\mathrm{PAH}}<0.75 \%$, while $30 / 61$ of the galaxies have $q_{\mathrm{PAH}}>3.25 \%$.

Roche et al. (1991) noted that the $8-13 \mu \mathrm{m}$ spectra of AGNs were often deficient in the PAH emission features commonly seen in $\mathrm{H}$ II region galaxies. The spectrum of PAH emission

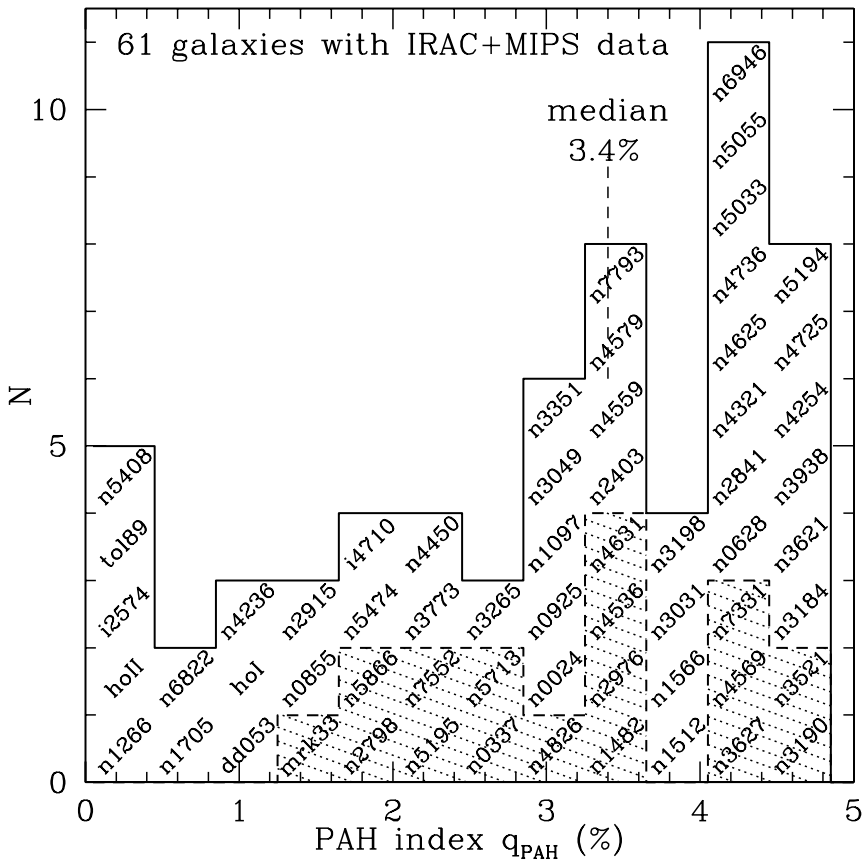

FIG. 19.-Shaded histogram: the 17 galaxies with SCUBA data for which we can estimate the PAH index $q_{\mathrm{PAH}}$. The solid histogram includes 44 additional galaxies without SCUBA fluxes for which $q_{\mathrm{PAH}}$ can be estimated. Four galaxies for which $q_{\text {PAH }}$ cannot be determined have been omitted (see text).

around AGNs has since been found to often exhibit unusual band ratios, with some AGN systems showing extremely low values of $L(7.7 \mu \mathrm{m}) / L(11.3 \mu \mathrm{m})$ (Smith et al. 2007). The suppression of $7.7 \mu \mathrm{m}$ emission in AGNs may be attributable to destruction of small PAHs by, e.g., X-rays from the AGN (Voit 1992). Figure 20 shows the distribution of global values of $q_{\mathrm{PAH}}$ for SINGS galaxies with (a) low-luminosity AGNs (Seyfert or LINER) or (b) H II nuclei (the AGN/H II nuclear classification is from J. Moustakas et al. 2007, in preparation). Only galaxies with characteristic metallicities $A_{\mathrm{O}}>8.1$ are included. The galaxies with AGNs tend

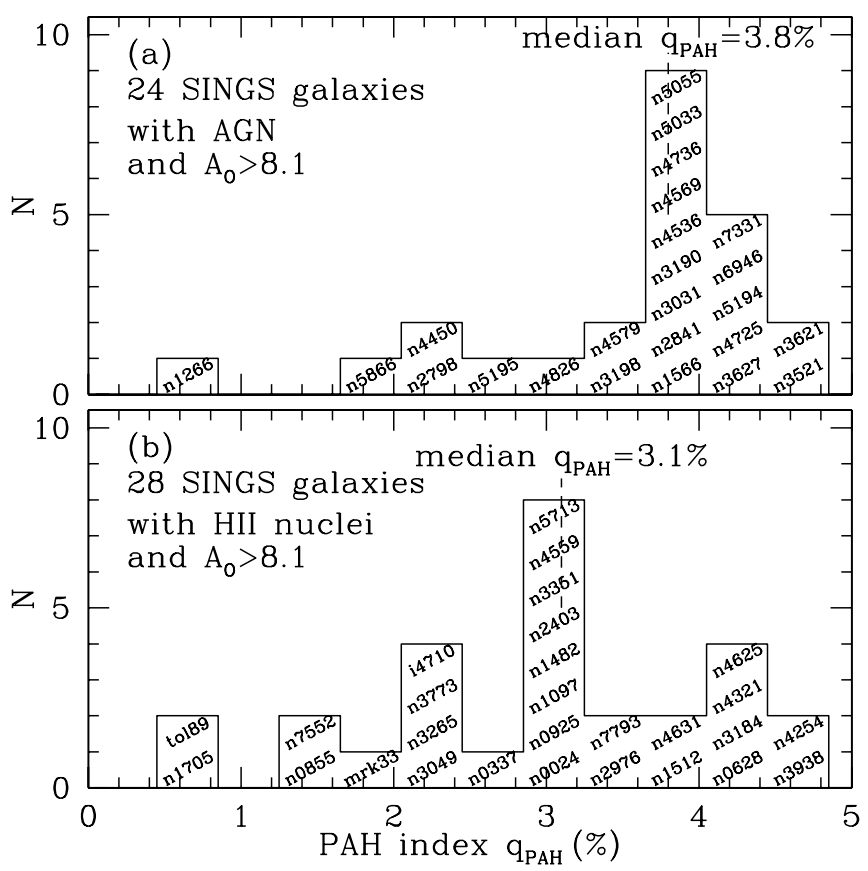

FIG. 20.- Histogram of global $q_{\mathrm{PAH}}$ for SINGS galaxies with $A_{\mathrm{O}}>8.1$, (a) with and $(b)$ without an AGN component. 


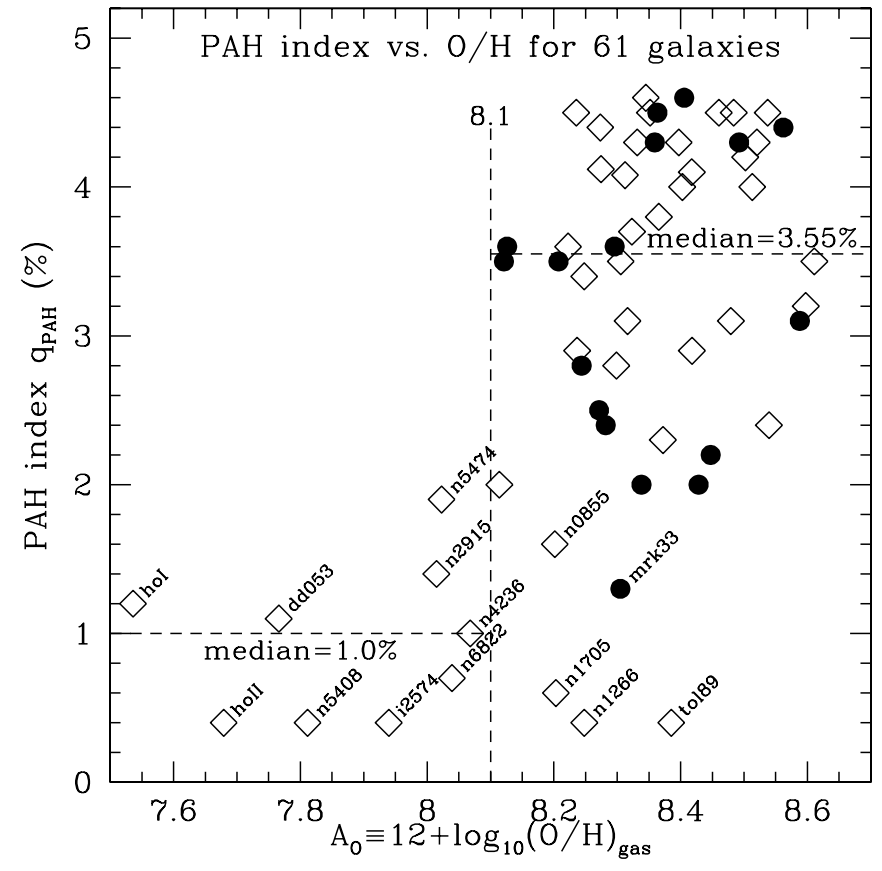

FIG. 21.-PAH index (percentage of dust mass contributed by PAHs with $N_{\mathrm{C}}<10^{3} \mathrm{C}$ atoms) vs. galaxy metallicity (see text). Low-metallicity galaxies always have low PAH index $q_{\mathrm{PAH}}$. Filled circles are SINGS-SCUBA galaxies; diamonds are SINGS galaxies lacking submillimeter data. [See the electronic edition of the Journal for a color version of this figure.]

to have higher values of $q_{\mathrm{PAH}}$ than the $\mathrm{H}$ II galaxies: the global SEDs show no evidence for PAH suppression. This is presumably because the SINGS sample excludes powerful AGNs, and lowluminosity AGNs have little effect on the global SED. Note that the five galaxies in Figure $20 b$ with $q_{\mathrm{PAH}}<2 \%$ include two irregulars (NGC 1705, Mrk 33), one elliptical (NGC 0855), and a dwarf starburst ( Tol 89).

Figure 21 shows $q_{\text {PAH }}$ versus metallicity for the 61 galaxies in the present study for which $q_{\mathrm{PAH}}$ has been determined. We are able to estimate $q_{\text {PAH }}$ for galaxies spanning a range of at least 0.9 dex in metallicity, from $A_{\mathrm{O}}=7.68 \pm 0.03$ (Holmberg II) to $8.60 \pm 0.06(\mathrm{NGC} 3351) .\left(A_{\mathrm{O}}=7.54 \pm 0.34\right.$ of Holmberg $\mathrm{I}$ is nominally lower than for Holmberg II but is uncertain.) A clear separation is seen: all nine of the galaxies with $A_{\mathrm{O}}<8.1$ have $q_{\text {PAH }} \leq 1.9 \%$, with median $q_{\text {PAH }}=1.0 \%$. When $A_{\mathrm{O}} \gtrsim 8.1$, higher PAH abundances are seen, with median $q_{\mathrm{PAH}}=3.55 \%$ for the 52 galaxies with $A_{\mathrm{O}}>8.1$, although note that only 3 of the 52 galaxies with $A_{\mathrm{O}}>8.1$ have $q_{\mathrm{PAH}}<1.0 \%$ (the median for $A_{\mathrm{O}}<8.1$ ). $\mathrm{PAH}$ emission strength, as a fraction of total IR emission, therefore appears to always be low for $A_{\mathrm{O}}<8.1$.

The weakness of PAH emission from low-metallicity galaxies was first noted by Roche et al. (1991) using ground-based spectroscopy and further investigated with ISO data by Boselli et al. (1998), Sturm et al. (2000), and Madden (2000). The SMC, with $A_{\mathrm{O}}=8.0$ (Kurt \& Dufour 1998; Garnett 1999), falls just below the apparent threshold $A_{\mathrm{O}}=8.1$ seen in Figure 21. Li \& Draine (2002) concluded that the SED of the SMC Bar was consistent with the "SMC" dust model of WD01 with a PAH index $q_{\mathrm{PAH}}$ of only $0.1 \%$ (see Table 3 ). ${ }^{20}$ ISO observed PAH emission from molecular cloud SMC B1 No. 1 (Reach et al. 2000) outside the SMC Bar; Li \& Draine (2002) showed that the observed emis-

20 This result is controversial: for the SMC Bar, Bot et al. (2004) have argued that the PAH abundance as a fraction of the dust mass is similar to the MW value, which we estimate to correspond to $q_{\mathrm{PAH}} \approx 5 \%$. Observations of the SMC with Spitzer should soon clarify this issue. sion could be reproduced with a model with only $3 \%$ of the SMC carbon in PAHs, corresponding to $q_{\mathrm{PAH}} \approx 0.8 \%$. Thus, the SMC appears to have $q_{\text {PAH }}<1 \%$.

Hunt et al. (2005) observed PAH emission to be weak in a number of low-metallicity blue compact dwarf galaxies. In a photometric study of 34 galaxies spanning two decades in metallicity, Engelbracht et al. (2005) concluded that there was a sharp difference in the ratio of $8 \mu \mathrm{m}$ emission from PAHs to $24 \mu \mathrm{m}$ emission from warm dust for metallicities above and below a threshold value $A_{\mathrm{O}} \approx 8.2 .^{21}$ Hogg et al. (2005) compared PAH emission with starlight for a sample of 313 SDSS galaxies and noted that low-luminosity (presumably low metallicity) star-forming galaxies tended to have low ratios of PAH emission to starlight. Using ISOCAM data, Madden et al. (2006) concluded that PAH abundance, relative to larger grains, was positively correlated with metallicity and suppressed in systems with hard radiation fields (i.e., high [ $\mathrm{Ne}$ III]/[Ne II] ratio). In a spectroscopic study of blue compact dwarf galaxies, Wu et al. (2006) found that the equivalent width of the PAH 6.2 and $11.2 \mu \mathrm{m}$ features appeared to be suppressed for $Z / Z_{\odot} \lesssim 0.2$, or $A_{\mathrm{O}} \lesssim 7.9$.

The threshold $A_{\mathrm{O}}=8.1$ found here is close to the value 8.2 found by Engelbracht et al. (2005) and the value 8.0 found by $\mathrm{Wu}$ et al. (2006). The reason for the small value of $q_{\text {PAH }}$ when $A_{\mathrm{O}}<8.1$ is not clear at this time. A number of possibilities exist:

1. Low $A_{\mathrm{O}}$ might imply more rapid destruction of PAHs by $\mathrm{UV}$ radiation in an ISM with reduced shielding by dust.

2. Low $A_{\mathrm{O}}$ might imply more effective destruction of PAHs by thermal sputtering in shock-heated gas that cools slowly because of the reduced metallicity.

3. Galaxies with low $\mathrm{O} / \mathrm{H}$ have lower values of $\mathrm{C} / \mathrm{O}$ (Henry $\&$ Worthey 1999); the reduced PAH abundance in these galaxies might be due to a deficiency of PAH-producing carbon stars and C-rich planetary nebulae.

4. PAH formation and growth in the ISM would be suppressed by low gas-phase $\mathrm{C}$ abundances.

5. We also call attention to the relatively low value of $q_{\mathrm{PAH}} \approx$ $1.3 \%$ for Mrk 33, which with $A_{\mathrm{O}}=8.30 \pm 0.10$ (J. Moustakas et al. 2007, in preparation) is well above the threshold $A_{\mathrm{O}} \approx 8.1$. The relatively low value of $q_{\mathrm{PAH}}$ in Mrk 33 may be a consequence of the vigorous star formation in Mrk 33, which, via the combined effects of hard UV and supernova blast waves, may be enhancing the rate of destruction of PAHs, thus acting to lower the steady state PAH abundance.

6. The strong $7.6 \mu \mathrm{m}$ emission feature requires free-flying PAHs with $N_{\mathrm{C}}<10^{3} \mathrm{C}$ atoms (DL07), and emission can therefore be suppressed if small PAHs coagulate with larger grains. Such coagulation can take place in molecular clouds. (How this might correlate with metallicity is uncertain, as the metallicity dependence of molecular cloud properties is not understood.)

While we can speculate about various effects that may be important, our limited understanding of the dynamics of the ISM in other galaxies, together with our limited knowledge regarding many of the processes acting to form and destroy PAHs in the ISM, precludes definite explanations of the observed galaxy-togalaxy variations in $q_{\mathrm{PAH}}$.

\section{STARLIGHT PROPERTIES FOR 65 SINGS GALAXIES}

The distribution of $U_{\min }$ values is shown in Figure 22. The shaded portion of the histogram is contributed by the 17 galaxies

\footnotetext{
21 Note that the oxygen abundances used by Engelbracht et al. (2005) were based on a heterogeneous compilation of measurements from the literature, whereas the abundances in our study have been derived self-consistently and placed on a common abundance scale (for details see J. Moustakas et al. 2007, in preparation).
} 


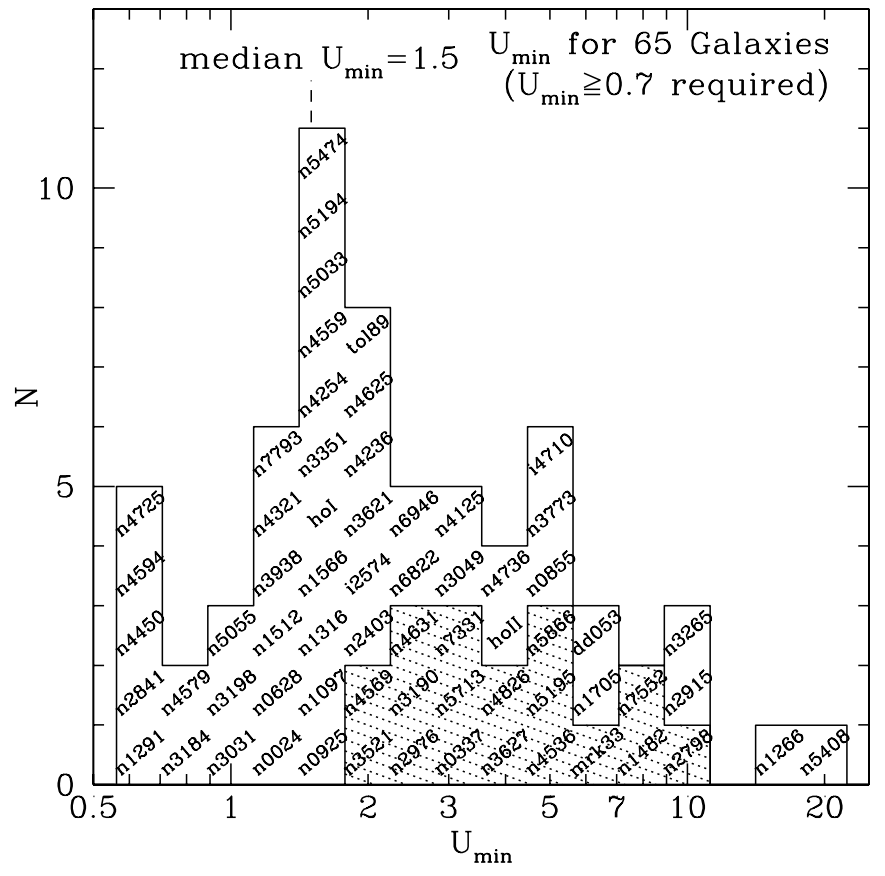

FIG. $22 a$

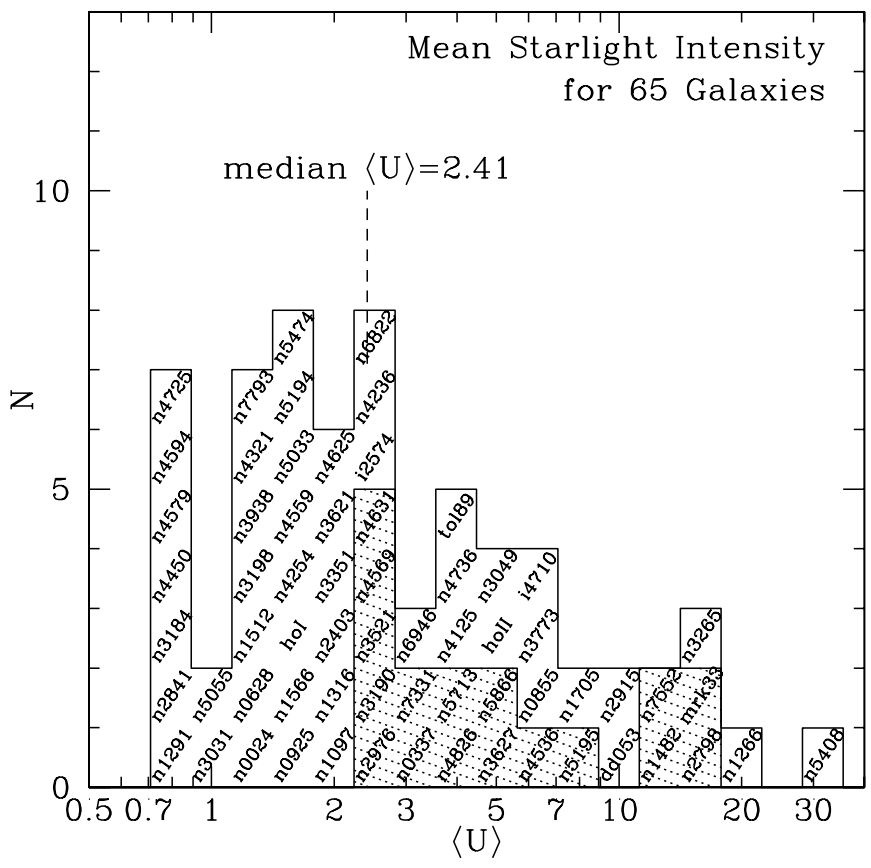

FIG. $22 b$

FIG. 22. $-U_{\min }$ and $\langle U\rangle$ for 65 galaxies (see text). The shaded portion of the histogram consists of the 17 galaxies for which SCUBA global fluxes constrain the dust models. [See the electronic edition of the Journal for a color version of this figure.]

for which SCUBA data were available and used in the model fitting with fixed $U_{\max }=10^{6}$; for these galaxies, there was no lower limit imposed on $U_{\min }$. Two of the 17 galaxies have $U_{\min }=$ 2.0 , and the other 15 have $U_{\min } \geq 2.5$. The remaining 48 galaxies lack submillimeter data, and for these the restricted fitting pro- cedure allows only $U_{\min } \geq 0.7$. This lower limit on $U_{\min }$ results in a small "pileup" of five galaxies at $U_{\min }=0.7$ in Figure 22; possibly some of these should have smaller values of $U_{\min }$. What is needed, of course, are submillimeter observations so that the restricted fitting procedure becomes unnecessary.

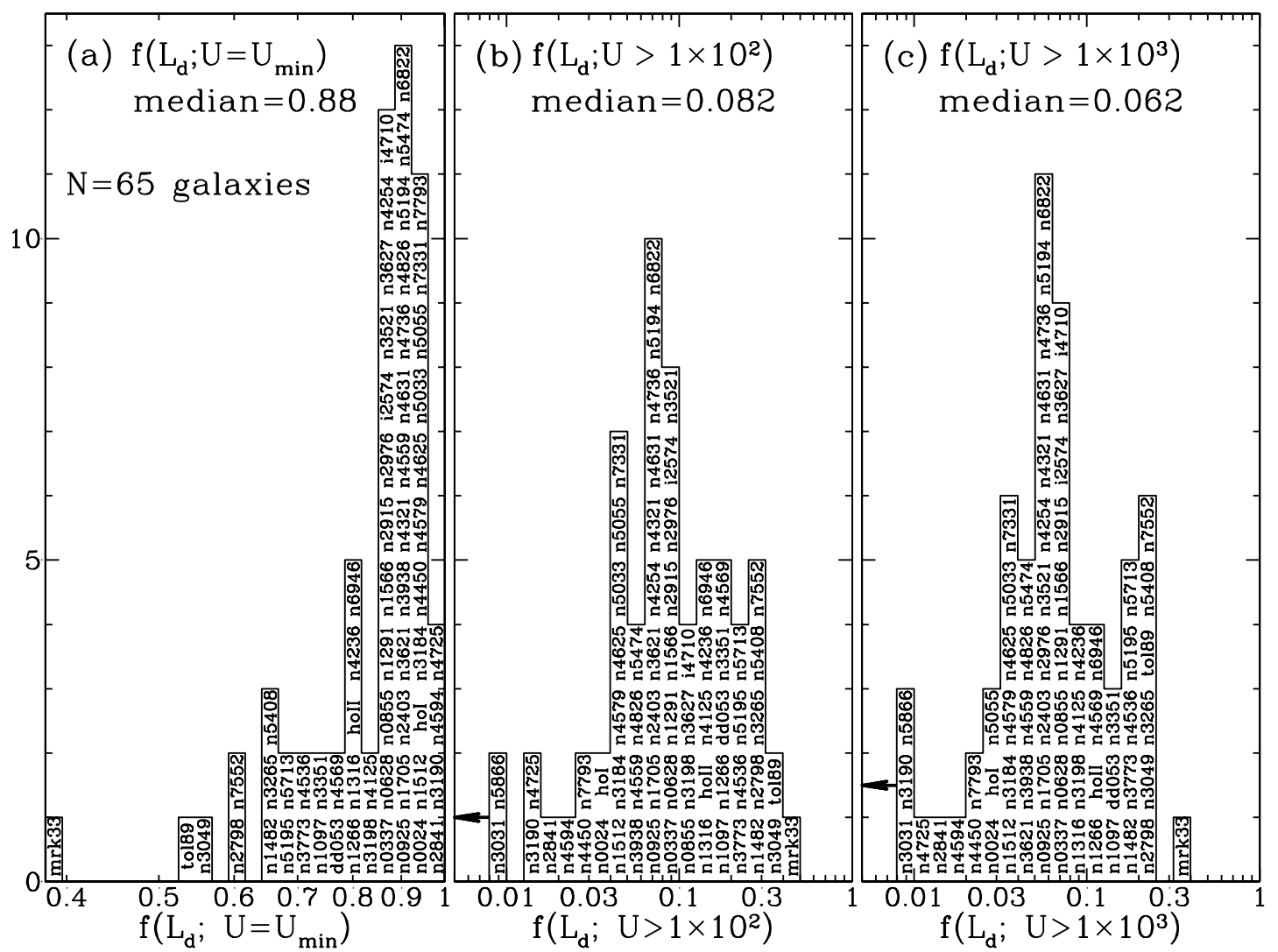

FIG. 23. - Fraction of the dust luminosity originating in regions with $(a) U=U_{\min },(b) U>10^{2}$, and $(c) U>10^{3}$ (see text). Galaxies with $f \leq 0.01$ have been grouped in a bin at 0.01 . 
The form of the starlight distribution function (eq. [6]) is based on the expectation that the bulk of the IR luminosity would be radiated by grains in a diffuse ISM with a more or less uniform starlight background, with $U \approx U_{\min }$. This assumption appears to be validated by the success of the model in reproducing the observed dust emission extending from 5.7 to $850 \mu \mathrm{m}$. It is interesting that the median $U_{\min }=1.5$ is only slightly more intense than our local ISRF $(U=1)$, suggesting that interstellar processes (star formation, absorption of starlight by dust) regulate the diffuse starlight intensity to have $U \approx 1.5$ to within a factor of 2 .

The distribution of mean starlight intensities is shown in Figure 22b. As before, the galaxies with SCUBA data are shaded. The galaxies with SCUBA data and those without SCUBA data both show a tendency to have $\langle U\rangle \approx 2.5$, although about $12 \%$ of the sample has $\langle U\rangle>10$.

The contribution of high-intensity regions to the dust heating is shown in Figure 23. In many of the sample galaxies, the observed $24 \mu \mathrm{m}$ emission appears to require that a significant fraction of the dust heating take place in regions with high starlight intensities. Figure 23 shows that the median galaxy has $\sim 8 \%$ of the total IR power originating from regions with $U>10^{2}$ and $\sim 6 \%$ of the IR power originating from regions with $U>10^{3}$. In most cases these high-intensity regions are probably PDRs bounding dense $\mathrm{H}$ II regions. For example, the dust just outside the ionization front in the Orion Bar PDR is heated by starlight with $U \approx 3 \times 10^{4}$ (Allers et al. 2005), and similar regions should be present in other star-forming galaxies. For steady star formation with the initial mass function observed in the MW, perhaps $\sim 10 \%$ of the bolometric luminosity is contributed by the massive $\left(M \gtrsim 20 M_{\odot}\right)$ stars that tend to spend most of their luminous existences close to their natal clouds, photodissociating and photoionizing the gas, and heating the dust. Therefore, the median galaxies in Figure 23 do not seem surprising.

The galaxies with the highest values of $f\left(U>10^{3}\right)$ are Mrk 33 (Im), Tol 89 (Im), NGC 5408 (Im), NGC 2798 (Sa), NGC 3049 (Sab), and NGC 7552 (Sab). All of these galaxies appear to be forming stars at a high rate per unit area (see, e.g., discussion of Mrk 33 in $\S 5.6 .2)$, and all have high ratios of $\left(\nu F_{\nu}\right)_{24 \mu \mathrm{m}} /$ $\left(\nu F_{\nu}\right)_{160 \mu \mathrm{m}}$, ranging from 0.52 for Tol 89 to 1.47 for Mrk 33, indicative of a substantial fraction of the dust power originating in regions where the grains are hot enough to radiate at $24 \mu \mathrm{m}$.

DL07 showed that, for their dust models, the fraction of the starlight heating contributed by high-intensity regions could be related to the observed quantity

$$
P_{24}-0.14 P_{7.9} \equiv \frac{\left\langle\nu F_{\nu}^{\mathrm{ns}}\right\rangle_{24}-0.14\left\langle\nu F_{\nu}^{\mathrm{ns}}\right\rangle_{7.9}}{\left\langle\nu F_{\nu}\right\rangle_{71}+\left\langle\nu F_{\nu}\right\rangle_{160}}
$$

where $F_{\nu}^{\text {ns }}$ is the nonstellar (i.e., dust) contribution to the flux density. The term $0.14\left\langle\nu F_{\nu}^{\mathrm{ns}}\right\rangle_{7.9}$ in the numerator of equation (22) is intended to approximate the contribution to the $24 \mu \mathrm{m}$ flux arising from single-photon heating of PAHs, so that the numerator of equation (22) is the $24 \mu \mathrm{m}$ flux contributed by larger grains, which will only radiate at $24 \mu \mathrm{m}$ when exposed to high-intensity starlight. Figure 24 shows the estimated fraction of the dust power originating in regions with $U>10^{2}$ versus the observed value of the quantity $\left(P_{24}-0.14 P_{7.9}\right)$. The galaxies fall in an approximately one-dimensional sequence, and the fraction of the starlight absorption taking place in high-intensity PDRs can be estimated from the observed $\left\langle F_{\nu}^{\text {ns }}\right\rangle_{7.9},\left\langle F_{\nu}^{\text {ns }}\right\rangle_{24},\left\langle F_{\nu}\right\rangle_{71}$, and $\left\langle F_{\nu}\right\rangle_{160}$ using the DL07 fitting function

$$
f\left(L_{d} ; U>10^{2}\right) \approx 1.05\left(P_{24}-0.14 P_{7.9}-0.035\right)^{0.75} .
$$

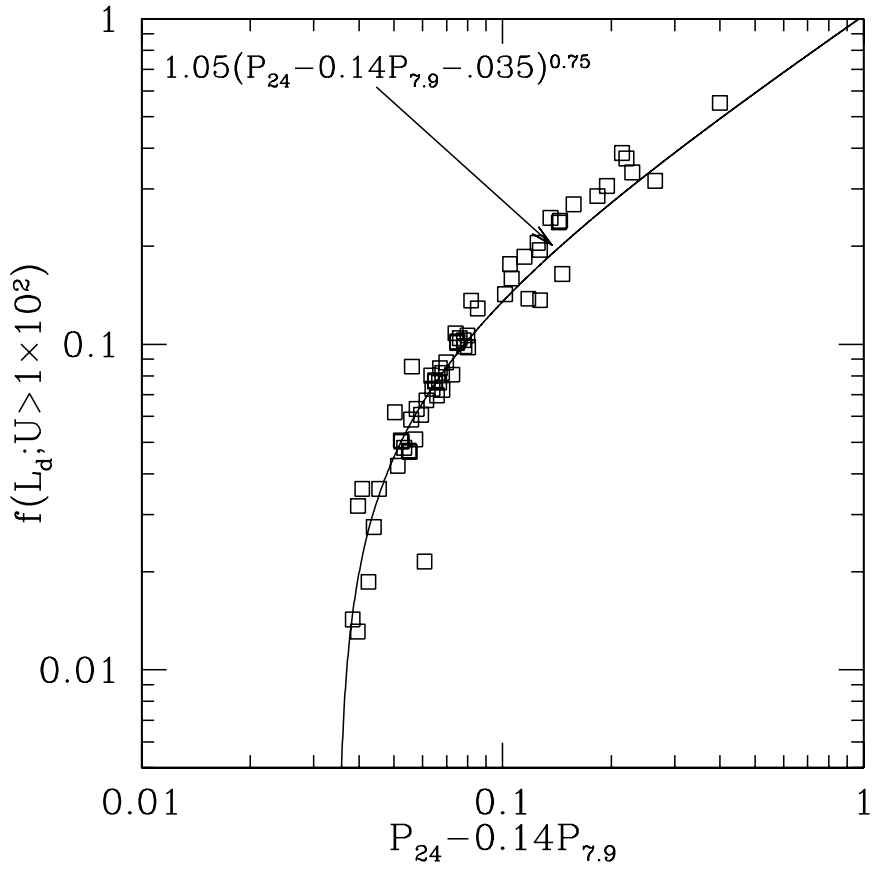

FIG. 24.-Estimated fraction of the dust luminosity contributed by dust in regions with $U>10^{2}$ vs. observed value of $P_{24}-0.14 P_{7.9}$ (see text). Also shown is the fitting function proposed by DL07.

\section{DISCUSSION}

\subsection{What About the Dust in Dark Clouds?}

The adopted "model" for the distribution of starlight intensities makes no allowance for the possibility of dust in dark clouds, since it assumes that there is no dust heated by starlight intensities below the "diffuse ISM" value $U_{\min }$. While such dust may not be important as a contributor to the total bolometric luminosity, it would not be surprising if it contained an appreciable fraction of the total dust mass, and it might have been expected to contribute an appreciable fraction of the emission at $850 \mu \mathrm{m}$.

However, we have seen that dust with the physical properties adopted by DL07 appears to reproduce the observed SEDs of the 17 SINGS-SCUBA galaxies without a "cold dust" component; indeed, when we fix $U_{\max }=10^{6}$, the best-fit models for the 17 SINGS-SCUBA galaxies all have $U_{\min } \geq 2$.

As discussed by DL07, the dust emissivities employed here reproduce the FIR and submillimeter emission from our local diffuse ISM at Galactic latitudes $|b|>7^{\circ}$ as measured by the FIRAS instrument on COBE (Finkbeiner et al. 1999). To the extent that our models reproduce the observed global FIR and submillimeter emission from other galaxies, it suggests that the dust in dark clouds makes only a minor contribution to the emission. Suppose that a fraction $f_{\text {cold }}$ of the dust mass is actually in dark clouds, with dust temperature $T_{\text {cold }}$, and the remainder $\left(1-f_{\text {cold }}\right)$ is in diffuse clouds, all at a single dust temperature $T_{\text {diff }}$. Then the fraction $p_{\text {cold }}$ of the total emission contributed by the dark cloud dust at wavelength $\lambda$ is just

$$
p_{\text {cold }}(\lambda)=\frac{f_{\text {cold }} /\left(e^{x_{c}}-1\right)}{f_{\text {cold }} /\left(e^{x_{c}}-1\right)+\left(1-f_{\text {cold }}\right) /\left(e^{x_{d}}-1\right)},
$$

where $x_{c} \equiv h c / \lambda k T_{\text {cold }}$ and $x_{d} \equiv h c / \lambda k T_{\text {diff }}$. If we take $T_{\text {diff }}=$ $20 \mathrm{~K}$ and $T_{\text {cold }}=12 \mathrm{~K}$, for example, then at $850 \mu \mathrm{m}$ we have

$$
p_{\text {cold }}=\frac{f_{\text {cold }}}{f_{\text {cold }}+2.33\left(1-f_{\text {cold }}\right)} .
$$


TABLE 7

Comparison with Results of Graphical Method

\begin{tabular}{|c|c|c|c|c|c|c|}
\hline \multirow[b]{2}{*}{ GALAXY } & \multicolumn{2}{|c|}{$q_{\mathrm{PAH}}(\%)$} & \multicolumn{2}{|c|}{$\log \left(M_{\text {dust }} / M_{\odot}\right)$} & \multicolumn{2}{|c|}{$\langle U\rangle$} \\
\hline & $\mathrm{GM}^{\mathrm{a}}$ & SEDM $^{\mathrm{b}}$ & $\mathrm{GM}^{\mathrm{a}}$ & SEDM $^{\mathrm{b}}$ & $\mathrm{GM}^{\mathrm{a}}$ & SEDM $^{\mathrm{b}}$ \\
\hline 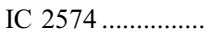 & 0.5 & 0.4 & 5.79 & 5.86 & 2.6 & 2.3 \\
\hline Mrk 33 ................. & 3.2 & 1.3 & 6.41 & 6.47 & 18 & 16 \\
\hline NGC $1266 \ldots \ldots \ldots . . .$. & 0.5 & 0.4 & 7.04 & 7.05 & 16 & 18 \\
\hline NGC $3521 \ldots \ldots \ldots \ldots . .$. & 4.6 & 4.5 & 8.10 & 7.84 & 1.2 & 2.2 \\
\hline NGC $6822 \ldots \ldots \ldots . . .$. & 1.1 & 0.7 & 5.15 & 5.04 & 2.1 & 2.8 \\
\hline
\end{tabular}

${ }^{a}$ Graphical method from DL07.

${ }^{\mathrm{b}}$ Results from fitting MW models with $U_{\max }=10^{6}$ and $U_{\min } \geq 1$.

Hence, if, hypothetically, $50 \%$ of the dust mass were in the cold clouds ( $f_{\text {cold }}=0.5$ ), they would contribute only $30 \%$ of the total emission at $850 \mu \mathrm{m}$; if this hypothetical cold component were left out, the model would fall short at $850 \mu \mathrm{m}$ by only $30 \%$, comparable to the existing photometric uncertainties.

Because the model does not include a cold dust component, it would not be surprising if the resulting estimates of the dust mass $M_{\text {dust }}$ were low. However, we have seen that if we adopt $X_{\mathrm{CO}}=4 \times 10^{20} \mathrm{~cm}^{-2}\left(\mathrm{~K} \mathrm{~km} \mathrm{~s}^{-1}\right)^{-1}$, we obtain dust-to-gas mass ratios that appear to account for all of the refractory elements in the ISM, as shown in Figure 17. If in fact we have substantially underestimated the dust mass in some of these galaxies, then our adopted gas masses would have to also be increased, by using an even larger value of $X_{\mathrm{CO}}$.

Note that while much of the gas in the MW is molecular, much of this molecular gas is actually in regions with visual extinction $A_{V} \lesssim 1$, so that it is not "dark" and the dust grains are heated by diffuse starlight. In dark clouds that are star-forming, such as Orion OMC-1 or M17, much of the dust is actually warm, heated by the embedded stars. It appears most likely that the fraction of the dust mass that is genuinely "cold" is probably relatively small (less than, say, 30\%). Although the model omits a cold dust component, it appears to recover the majority of the global dust mass in the SINGS galaxies.

\subsection{Comparison with Graphical Methods}

The model fitting carried out here has been done by an automated fitting procedure, which includes using interpolated models with values of $q_{\mathrm{PAH}}$ intermediate between the seven $q_{\mathrm{PAH}}$ values in the MW dust models examined by DL07. DL07 presented a straightforward graphical procedure for estimating $q_{\mathrm{PAH}}$, dust masses, and starlight intensities from IRAC and MIPS photometry and applied it to five galaxies as examples. The results of that graphical procedure are compared with the present results in Table 7. For two of the galaxies (Mrk 33 and NGC 3521), the SED model fitting made use of SCUBA data, which are not used in the DL07 graphical procedure.

Table 7 shows generally good agreement between the graphical method and the present method for estimation of $q_{\mathrm{PAH}}$, $M_{\text {dust }}$, and $\langle U\rangle$. The dust masses agree to within a factor of 2 in all cases. The values of $q_{\mathrm{PAH}}$ are in generally good agreement except for Mrk 33, where $q_{\mathrm{PAH}}=3.2 \%$ estimated from the graphical method is considerably larger than $q_{\mathrm{PAH}}=1.3 \%$ from the SED fitting procedure used in this paper. The reason for this discrepancy is straightforward: the DL07 graphical fitting procedure advises the user to estimate $q_{\mathrm{PAH}}$ by seeking a model that reproduces the $7.9 \mu \mathrm{m}$ emission with the smallest possible value of $\gamma$, the fraction of the dust mass exposed to starlight with $U>U_{\min }$. While this approach is appropriate for most galaxies, which generally have $\gamma \lesssim 0.03$, Mrk 33 has an extreme spectrum, with a very large fraction of the dust exposed to intense radiation fields (see the location of Mrk 33 in Fig. 23). The SED modeling in the present paper estimates $\gamma=0.12$ for Mrk 33. In fact, examination of the graphs shown in DL07 (Figs. 20 and 21 of that paper) reveals that a solution with $\gamma \approx 0.1$ does provide a better fit to the observed flux ratios. The DL07 graphical fitting procedure should therefore be employed with caution for galaxies with $P_{24} \equiv\left\langle\nu F_{\nu}^{\mathrm{ns}}\right\rangle_{24} /\left[\left\langle\nu F_{\nu}\right\rangle_{71}+\left\langle\nu F_{\nu}\right\rangle_{160}\right] \gtrsim 0.2$, indicative of substantial amounts of IR power radiated by dust in intense radiation fields.

\subsection{Dust Composition}

The graphite component of the dust model used here produces a broad emission feature near $33 \mu \mathrm{m}$ when the dust is illuminated by starlight with $U \gtrsim 10^{4}$ (DL07). This feature is a consequence of the optical properties assumed for graphite.

Visual inspection of the $25-35 \mu \mathrm{m}$ spectra presented by Smith et al. (2007) suggests that the $33 \mu \mathrm{m}$ feature is not present, at least not with the strength that would be expected in some of our global models. Some of the central spectra (e.g., NGC 4321) have a broad $(\mathrm{FWHM} \approx 1.7 \mu \mathrm{m}$ ) emission feature near $33 \mu \mathrm{m}$, but it is weak ${ }^{22}$ and narrower than the very broad (FWHM $\approx 20 \mu \mathrm{m}$ ) peak found in our models. A similar $\sim 1 \mu$ m wide plateau near 33-34 $\mu \mathrm{m}$ was also seen in the ISO spectra of bright starburst galaxies (Sturm et al. 2000) and planetary nebulae (Waters et al. 1998) and tentatively attributed to crystalline olivine. If future observations find that the graphite feature is not present with the strength predicted by our model calculations, what would be the implications? First of all, it must be remembered that the present calculations are based on two highly idealized assumptions: (1) the $a>100 \AA$ carbonaceous grains are spherical and composed of single crystals of graphite, and (2) the dielectric tensor in the IR conforms to a simple free-electron model (Draine \& Lee $1984,1987)$. The $33 \mu \mathrm{m}$ feature is a consequence of the dielectric function for $\boldsymbol{E} \| c$, which is directly related to the electrical conductivity parallel to the $c$-axis (i.e., normal to the graphite basal plane). This conductivity is modeled by a free electron gas with a plasma frequency $\omega_{p}=1.53 \times 10^{14} \mathrm{~s}^{-1}$ and a damping time $\tau=$ $1.4 \times 10^{-14} \mathrm{~s}$. The simple free-electron model is not expected to accurately describe the detailed wavelength dependence of this feature (located at $\omega=2 \pi c / 33 \mu \mathrm{m}=5.7 \times 10^{13} \mathrm{~s}^{-1}$ ). Furthermore, even if the model were accurate for ideal graphite, it seems likely that impurities and defects, which might vary from grain to grain in a single region or from region to region in the galaxy, would have the effect of causing $\omega_{p}$ and $\tau$ to vary over the population of emitting grains (and perhaps even within a single grain), resulting in further broadening of this feature. Therefore, failure to observe this feature would probably not rule out graphite as a grain constituent; it would only rule out monocrystalline graphite grains with a dielectric function resembling the simple free-electron model used by Draine \& Lee (1984).

Of course, it would not be surprising if the carbonaceous component of the interstellar grain population consisted of something quite different from monocrystalline graphite. "Amorphous carbon," for instance, refers to a class of materials with widely varying properties: for example, the amorphous carbon materials BE, ACAR, and ACH2 studied by Colangeli et al. $(1993,1995)$ and Mennella et al. (1995) are all insulators, with bandgaps of $0.15,0.52$, and $1.32 \mathrm{eV}$, respectively (Zubko et al. 1996). These carbon solids, each having different properties in the FIR, would

\footnotetext{
22 The PAHFIT spectrum decomposition program (Smith et al. 2007) assigns an equivalent width of $\sim 0.2 \mu \mathrm{m}$ for these galaxies.
} 
not show the $33 \mu \mathrm{m}$ feature. It is quite possible that the carbonaceous material in the larger $(a \gtrsim 0.02 \mu \mathrm{m})$ interstellar grains may be better approximated by some form of amorphous carbon. However, we have seen that the SINGS photometry appears to also be compatible with graphite.

\subsection{The $\mathrm{H}_{2} / \mathrm{CO}$ Conversion Factor $X_{\mathrm{CO}}$}

To estimate the dust-to-gas mass ratio in $\S 7.1$, we need the total hydrogen mass $M_{\mathrm{H}}$, including $\mathrm{H}_{2}$. Because $\mathrm{H}_{2}$ resides primarily in its lowest two rotational states $(J=0$ and 1$)$, which do not radiate, the $\operatorname{CO} J=1 \rightarrow 0$ line is routinely employed to trace $\mathrm{H}_{2}$. It is usually assumed that the $\mathrm{H}_{2}$ column density is proportional to the CO 1-0 surface brightness (see eq. [14]), with an empirical conversion factor $X_{\mathrm{CO}}$ that is based on some method of estimating the total gas mass. For the MW, virial mass estimates based on GMC line widths give $X_{\mathrm{CO}}=3.0 \times$ $10^{20} \mathrm{~cm}^{-2}\left(\mathrm{~K} \mathrm{~km} \mathrm{~s}^{-1}\right)^{-1}$ (Young \& Scoville 1991). Studies using $\gamma$-ray emission as a mass tracer in the MW found $X_{\mathrm{CO}}=$ $(1.9 \pm 0.2) \times 10^{20} \mathrm{~cm}^{-2}\left(\mathrm{~K} \mathrm{~km} \mathrm{~s}^{-1}\right)^{-1}$ (Strong \& Mattox 1996) and $(1.56 \pm 0.05) \times 10^{20}$ (Hunter et al. 1997). Using IR emission from dust as a mass indicator, Dame et al. (2001) found $X_{\mathrm{CO}}=$ $(1.8 \pm 0.3) \times 10^{20} \mathrm{~cm}^{-2}\left(\mathrm{~K} \mathrm{~km} \mathrm{~s}^{-1}\right)^{-1}$ for gas at $5^{\circ}<|b|<$ $30^{\circ}$. Because (1) CO 1-0 emission is often optically thick, (2) CO may be subthermally excited in the outer layers of molecular clouds, and (3) photodissociation of $\mathrm{CO}$ results in low $\mathrm{CO} / \mathrm{H}_{2}$ ratios in the outer layers of molecular clouds, there is no reason to expect a single value of $X_{\mathrm{CO}}$ to apply to all galaxies, or to all regions within a galaxy.

For low-luminosity spiral galaxies in the Virgo Cluster, Boselli et al. (2002) estimated values of $X_{\mathrm{CO}}$ ranging from $\sim 10^{20} \mathrm{~cm}^{-2}$ $\left(\mathrm{K} \mathrm{km} \mathrm{s}^{-1}\right)^{-1}$ for giant spirals to $\sim 10^{21} \mathrm{~cm}^{-2}\left(\mathrm{~K} \mathrm{~km} \mathrm{~s}^{-1}\right)^{-1}$ for dwarf irregulars. Based on an extensive study of GMCs in a number of galaxies in the Local Group, Blitz et al. (2007) conclude that $X_{\mathrm{CO}}=4 \times 10^{20} \mathrm{~cm}^{-2}\left(\mathrm{~K} \mathrm{~km} \mathrm{~s}^{-1}\right)^{-1}$ is a good value to use for most galaxies if the individual GMCs are assumed to be virialized.

The present study has demonstrated that we can use a physical dust model to reproduce observed IR and FIR emission from nearby galaxies, allowing us to estimate the dust mass in these galaxies. Because we have a priori expectations for the value of the dust-to-gas mass ratio, our determination of $M_{\text {dust }}$ therefore allows us to estimate the total gas mass and thereby estimate $X_{\mathrm{CO}}$ for these galaxies.

Figure 16 explores the relationship between dust-to-gas mass ratio and metallicity. Observed values of $M_{\mathrm{dust}} / M_{\mathrm{H}}$ are plotted versus metallicity in Figures $16 a$ and $16 b$, showing only galaxies where both $\mathrm{H}$ I and $\mathrm{CO}$ have been detected, or where the upper limit on either $\mathrm{H}$ I or $\mathrm{CO}$ shows it to be of minor importance compared to the other gas component. Equation (13) is plotted in Figures $16 a$ and $16 b$, where we assume $\left[A_{\mathrm{O}}\right]_{\mathrm{MW}}=8.59$. The figure includes 12 galaxies for which SCUBA data constrain the dust models; these should be the most reliable dust masses. The figure includes an additional 20 galaxies lacking submillimeter data. In Figure $16 a$ the $\mathrm{H}_{2}$ mass is estimated using $X_{\mathrm{CO}}=$ $2 \times 10^{20} \mathrm{~cm}^{-2}\left(\mathrm{~K} \mathrm{~km} \mathrm{~s}^{-1}\right)^{-1}$, whereas Figure $16 b$ uses $X_{\mathrm{CO}}=$ $4 \times 10^{20} \mathrm{~cm}^{-2}\left(\mathrm{~K} \mathrm{~km} \mathrm{~s}^{-1}\right)^{-1}$.

From comparison of Figures $16 a$ and $16 b$, we see that $X_{\mathrm{CO}}=$ $4 \times 10^{20} \mathrm{~cm}^{-2}\left(\mathrm{~K} \mathrm{~km} \mathrm{~s}^{-1}\right)^{-1}$ results in dust-to-gas mass ratios for the SINGS-SCUBA galaxies that are consistent with the amount of material actually available to form silicate and carbonaceous grains, when one considers that the dust mass estimates are uncertain at the factor of 2 level and the metallicity estimates are also uncertain. There are some cases with mass determinations (not upper limits) that fall well above equation (13), which was expected to define an upper envelope for $M_{\mathrm{dust}} / M_{\mathrm{H}}$. For all cases where submillimeter data were used in the fitting, the dust mass estimates were expected to have better than factor of 2 accuracy; if we adopt $X_{\mathrm{CO}}=4 \times 10^{20} \mathrm{~cm}^{-2}\left(\mathrm{~K} \mathrm{~km} \mathrm{~s}^{-1}\right)^{-1}$, none of the galaxies with SCUBA data exceed equation (13) by more than a factor of 2. Conversely, $X_{\mathrm{CO}}=2 \times 10^{20} \mathrm{~cm}^{-2}\left(\mathrm{~K} \mathrm{~km} \mathrm{~s}^{-1}\right)^{-1}$ would imply dust masses for NGC 1482 and NGC 2976 that would be uncomfortably large given their estimated oxygen abundances. The one galaxy in Figure $16 b$ that falls well above the expected envelope is NGC 4594, the Sombrero galaxy. The ISM in this unusual system merits further study.

The histograms in Figures $16 c$ and $16 d$ show the distribution of the dust-to-gas mass ratio, normalized to the characteristic oxygen abundance of the MW. Ideally, the distribution would be narrow and peaking at $\sim 0.01$, possibly with a tail to low values of $\left(M_{\text {dust }} / M_{\mathrm{H}}\right) /\left[(\mathrm{O} / \mathrm{H}) /(\mathrm{O} / \mathrm{H})_{\mathrm{MW}}\right]$ representing galaxies where the grains do not incorporate a large fraction of the refractory elements. For $X_{\mathrm{CO}}=2 \times 10^{20} \mathrm{~cm}^{-2}\left(\mathrm{~K} \mathrm{~km} \mathrm{~s}^{-1}\right)^{-1}$, the median is at 0.016 . For $X_{\mathrm{CO}}=4 \times 10^{20} \mathrm{~cm}^{-2}\left(\mathrm{~K} \mathrm{~km} \mathrm{~s}^{-1}\right)^{-1}$, the median shifts to 0.012 , approximately the value expected if grains incorporate most of the refractory elements (as is the case in the MW).

The above considerations suggest that the value $X_{\mathrm{CO}} \approx 4 \times$ $10^{20} \mathrm{~cm}^{-2}\left(\mathrm{~K} \mathrm{~km} \mathrm{~s}^{-1}\right)^{-1}$ recommended by Blitz et al. (2007) for Local Group galaxies is also appropriate for the SINGS galaxy sample. This value of $X_{\mathrm{CO}}$ is a factor of $\sim 2$ larger than the value $(1.8 \pm 0.3) \times 10^{20} \mathrm{~cm}^{-2}\left(\mathrm{~K} \mathrm{~km} \mathrm{~s}^{-1}\right)^{-1}$ found recently by Dame et al. (2001) for the local MW.

\section{SUMMARY}

The principal results of this paper are as follows:

1. A physical dust model consisting of PAHs, carbonaceous grains, and amorphous silicate grains (DL07) heated by starlight, with a distribution of starlight intensities, successfully reproduces the IR and submillimeter emission for a sample of 17 galaxies with IRAC and MIPS photometry plus SCUBA photometry. In particular, the SCUBA photometry does not require an additional population of cold dust grains, or grains with enhanced submillimeter emissivities. Cold dust grains in dark clouds appear to contain only a minor fraction $(\$ 50 \%)$ of the total dust mass.

2 . The dust model allows the mean starlight intensity scale factor $\langle U\rangle$ and the total dust mass $M_{\text {dust }}$ to be estimated for each galaxy. For the 17 galaxies with SCUBA data, $\langle U\rangle$ ranges from 2.2 to 16 , with a median of 4.3 (see Fig. 7). For the 12 galaxies with SCUBA data and $\mathrm{CO}$ and $\mathrm{H}$ I observations, and assuming $X_{\mathrm{CO}}=4 \times 10^{20} \mathrm{~cm}^{-2}\left(\mathrm{~K} \mathrm{~km} \mathrm{~s}^{-1}\right)^{-1}$, the median $M_{\text {dust }} / M_{\mathrm{H}}=$ 0.0052 (see Fig. 4); this is similar to $M_{\text {dust }} / M_{\mathrm{H}} \approx 0.0073$ estimated for the MW based on observed depletions (see Table 2), or the value $\sim 0.010$ estimated from dust models that reproduce the observed MW extinction (see Table 3).

3. When submillimeter observations are unavailable, the mass of cool dust in a galaxy is poorly constrained. However, we use the model fits for the 17 galaxies where SCUBA data are available to test a "restricted" fitting procedure that can be applied when submillimeter observations are unavailable. For the 17 galaxies where global SCUBA data are available, the restricted fitting procedure recovered the dust mass to within a factor of 1.5 in $11 / 17$ cases (see Fig. 12) and to within a factor of 2.2 in every case. This restricted fitting procedure can therefore be used to estimate dust masses and starlight intensities in galaxies with dust emission detected in the three MIPS bands.

4. For the 20 galaxies that lack SCUBA fluxes but for which the gas mass is known, the median $M_{\text {dust }} / M_{\mathrm{H}} \approx 0.0088$ (see Fig. 15). 
5. By comparing dust-to-gas mass ratios computed with different assumed values of the $\mathrm{CO}$-to-molecular mass conversion factor $X_{\mathrm{CO}}$, we find that the dust-to-gas mass ratio normalized to galaxy metallicity gives dust masses consistent with available metal abundances for $X_{\mathrm{CO}} \approx 4 \times 10^{20} \mathrm{~cm}^{-2}\left(\mathrm{~K} \mathrm{~km} \mathrm{~s}^{-1}\right)^{-1}$ (see Fig. 16).

6. All 49 galaxies in Figure 17 with $A_{\mathrm{O}}>8.2$ appear to have dust masses consistent (to within a factor of $\sim 3$ ) with equation (13), corresponding to the total mass of dust in the WD01 models for MW dust (see Table 3) scaled by metallicity. This is close to the mass that will be formed if silicates are formed from the bulk of the interstellar $\mathrm{Mg}, \mathrm{Si}$, and $\mathrm{Fe}$, and $\sim 50 \%$ of the interstellar carbon goes into carbonaceous grains (see Table 2). All nine of the galaxies in Figure 17 with metallicities $A_{\mathrm{O}}<8.1$ appear to have global dust-to-gas ratios that fall below equation (13), in some cases by large factors: NGC 2915 appears to be at least a factor of 40 below equation (13). However, the dust-togas ratio in the regions where dust emission is detected appears to be close to equation (13). The low apparent global dust-to-gas ratios are found in dwarf galaxies with extended $\mathrm{H}$ I envelopes. When allowance is made for the lower metallicity and lower starlight intensity in the envelopes, it appears that the dust-to-gas mass ratios may be consistent with eq. (13).

7. We characterize the PAH abundance by a quantitative index $q_{\text {PAH }}$, defined to be the fraction of the total dust mass contributed by PAHs containing less than $10^{3} \mathrm{C}$ atoms. The 61 galaxies in this sample for which we are able to estimate $q_{\mathrm{PAH}}$ span the range of $\mathrm{PAH}$ index from $0.4 \%$ to $4.7 \%$, with a median $q_{\text {PAH }}=3.4 \%$ (see Fig. 19).
8. The PAH index $q_{\mathrm{PAH}}$ is strongly correlated with metallicity (see Fig. 21). The nine galaxies with $A_{\mathrm{O}}<8.1$ have $q_{\mathrm{PAH}} \leq 2 \%$ and median $q_{\mathrm{PAH}}=1.0 \%$, whereas the 52 galaxies with $A_{\mathrm{O}}>$ 8.1 have median $q_{\mathrm{PAH}}=3.55 \%$. Metallicity $A_{\mathrm{O}}=8.1$ appears to mark a transition in the composition of interstellar dust in galaxies, from low PAH to high PAH. Possible reasons for the observed variation in $q_{\mathrm{PAH}}$ are discussed in $\S 7.4$.

9. Except for three starbursting galaxies (Mrk 33, Tol 89, NGC 3049), the infrared emission from dust is dominated by dust grains exposed to starlight intensities $U \approx U_{\min }$, interpreted here as dust in diffuse regions heated by the general diffuse starlight background (see Fig. 23a). Half of the galaxies have more than $88 \%$ of the total dust power provided by this component.

10. The SEDs for the star-forming galaxies in the sample require that a significant fraction of the dust luminosity be produced by warm dust in regions of intense starlight. Half of the galaxies in the sample have more than $8 \%$ of the total dust luminosity originating in regions with $U>10^{2}$ (see Fig. 23). This appears to be consistent with the expected heating of dust in PDRs near massive stars.

We thank S. Madden and the anonymous referee for helpful comments. Support for this work, part of the Spitzer Space Telescope Legacy Science Program, was provided by NASA through the Jet Propulsion Laboratory under NASA contract 1407. B. T. D. was supported in part by NSF grant AST 04-06883.

B. T. D. is grateful to R. H. Lupton for availability of the SM graphics program.

\section{REFERENCES}

Ajhar, E. A., Lauer, T. R., Tonry, J. L., Blakeslee, J. P., Dressler, A., Holtzman, J. A., \& Postman, M. 1997, AJ, 114, 626

Allers, K. N., Jaffe, D. T., Lacy, J. H., Draine, B. T., \& Richter, M. J. 2005, ApJ, 630, 368

Bajaja, E., Krause, M., Wielebinski, R., \& Dettmar, R.-J. 1991, A\&A, 241, 411

Bakes, E. L. O., \& Tielens, A. G. G. M. 1994, ApJ, 427, 822

Becker, R., Mebold, U., Reif, K., \& van Woerden, H. 1988, A\&A, 203, 21

Bendo, G. J., et al. 2006a, ApJ, 645, 134 2006b, ApJ, 652, 283

Blitz, L., Fukui, Y., Kawamura, A., Leroy, A., Mizuno, N., \& Rosolowsky, E. 2007, in Protostars and Planets V, ed. B. Reipurth, D. Jewitt, \& K. Keil (Tucson: Univ. Arizona Press), 81

Boselli, A., Lequeux, J., \& Gavazzi, G. 2002, A\&A, 384, 33

Boselli, A., et al. 1998, A\&A, 335, 53

Bot, C., Boulanger, F., Lagache, G., Cambrésy, L., \& Egret, D. 2004, A\&A, 423, 567

Boulanger, F., \& Viallefond, F. 1992, A\&A, 266, 37

Cannon, J. M., et al. 2005, ApJ, 630, L37

2006a, ApJ, 647, 293

2006b, ApJ, 652, 1170

Cartledge, S. I. B., Lauroesch, J. T., Meyer, D. M., \& Sofia, U. J. 2004, ApJ, 613, 1037

Colangeli, L., Mennella, V., Blanco, A., Fonti, S., Bussoletti, E., Gumlich, H. E., Mertins, H. C., \& Jung, C. 1993, ApJ, 418, 435

Colangeli, L., Mennella, V., Palumbo, P., Rotundi, A., \& Bussoletti, E. 1995, A\&AS, 113, 561

Dale, D. A., Helou, G., Contursi, A., Silbermann, N. A., \& Kolhatkar, S. 2001, ApJ, 549, 215

Dale, D. A., et al. 2005, ApJ, 633, 857 2007, ApJ, 655, 863

Dame, T. M., Hartmann, D., \& Thaddeus, P. 2001, ApJ, 547, 792

Draine, B. T. 2003, ARA\&A, 41, 241

Draine, B. T., \& Lee, H. M. 1984, ApJ, 285, 89 1987, ApJ, 318, 485

Draine, B. T., \& Li, A. 2001, ApJ, 551, 807 2007, ApJ, 657, 810 (DL07)

Dumke, M., Krause, M., \& Wielebinski, R. 2004, A\&A, 414, 475

Dwek, E. 1998, ApJ, 501, 643

Edmunds, M. G. 2001, MNRAS, 328, 223
Engelbracht, C. W., Gordon, K. D., Rieke, G. H., Werner, M. W., Dale, D. A., \& Latter, W. B. 2005, ApJ, 628, L29

Fazio, G. G., et al. 2004, ApJS, 154, 10

Ferguson, A. M. N., Gallagher, J. S., \& Wyse, R. F. G. 1998, AJ, 116, 673

Finkbeiner, D. P., Davis, M., \& Schlegel, D. J. 1999, ApJ, 524, 867

Ford, H. C., Hui, X., Ciardullo, R., Jacoby, G. H., \& Freeman, K. C. 1996, ApJ, 458,455

Förster Schreiber, N. M., Roussel, H., Sauvage, M., \& Charmandaris, V. 2004, A\&A, 419, 501

Freedman, W. L., et al. 2001, ApJ, 553, 47

Galliano, F., Madden, S. C., Jones, A. P., Wilson, C. D., \& Bernard, J.-P. 2005 , A\&A, 434, 867

Galliano, F., Madden, S. C., Jones, A. P., Wilson, C. D., Bernard, J.-P., \& Le Peintre, F. 2003, A\&A, 407, 159

Garnett, D. R. 1999, in IAU Symp. 190, New Views of the Magellanic Clouds, ed. Y.-H. Chu et al. (Dordrecht: Kluwer), 266

Grevesse, N., \& Sauval, A. J. 1998, Space Sci. Rev., 85, 161

Habing, H. J. 1968, Bull. Astron. Inst. Netherlands, 19, 421

Heckman, T. M., Sembach, K. R., Meurer, G. R., Strickland, D. K., Martin, C. L., Calzetti, D., \& Leitherer, C. 2001, ApJ, 554, 1021

Helou, G., et al. 2004, ApJS, 154, 253

Henry, R. B. C., \& Worthey, G. 1999, PASP, 111, 919

Hirashita, H., Tajiri, Y. Y., \& Kamaya, H. 2002, A\&A, 388, 439

Hogg, D. W., Tremonti, C. A., Blanton, M. R., Finkbeiner, D. P., Padmanabhan, N., Quintero, A. D., Schlegel, D. J., \& Wherry, N. 2005, ApJ, 624, 162

Hony, S., Van Kerckhoven, C., Peeters, E., Tielens, A. G. G. M., Hudgins, D. M., \& Allamandola, L. J. 2001, A\&A, 370, 1030

Hunt, L., Bianchi, S., \& Maiolino, R. 2005, A\&A, 434, 849

Hunter, S. D., et al. 1997, ApJ, 481, 205

Izotov, Y. I., \& Thuan, T. X. 1998, ApJ, 500, 188

James, A., Dunne, L., Eales, S., \& Edmunds, M. G. 2002, MNRAS, 335, 753

Jenkins, E. B. 2004, in Origin and Evolution of the Elements, ed. A. McWilliam

\& M. Rauch (Cambridge: Cambridge Univ. Press), 336

Karachentsev, I. D., et al. 2002a, A\&A, 383, 125

. 2002b, A\&A, 385, 21

Kennicutt, R. C., Jr., et al. 2003, PASP, 115, 928

Kobulnicky, H. A., \& Kewley, L. J. 2004, ApJ, 617, 240

Krugel, E., Siebenmorgen, R., Zota, V., \& Chini, R. 1998, A\&A, 331, L9

Kurt, C. M., \& Dufour, R. J. 1998, Rev. Mex. AA Conf. Ser., 7, 202 
Leroy, A., Bolatto, A. D., Simon, J. D., \& Blitz, L. 2005, ApJ, 625, 763

Li, A., \& Draine, B. T. 2001, ApJ, 554, 778 (LD01) 2002, ApJ, 576, 762

Lisenfeld, U., \& Ferrara, A. 1998, ApJ, 496, 145

Madden, S. C. 2000, NewA Rev., 44, 249

Madden, S. C., Galliano, F., Jones, A. P., \& Sauvage, M. 2006, A\&A, 446, 877

Marconi, A., Testi, L., Natta, A., \& Walmsley, C. M. 1998, A\&A, 330, 696

Mathis, J. S., Mezger, P. G., \& Panagia, N. 1983, A\&A, 128, 212

Mennella, V., Colangeli, L., Blanco, A., Bussoletti, E., Fonti, S., Palumbo, P., \& Mertins, H. C. 1995, ApJ, 444, 288

Meurer, G. R. 1997, Publ. Astron. Soc. Australia, 14, 77

Meurer, G. R., Staveley-Smith, L., \& Killeen, N. E. B. 1998, MNRAS, 300, 705

Pagani, L., Lequeux, J., Cesarsky, D., Donas, J., Milliard, B., Loinard, L., \& Sauvage, M. 1999, A\&A, 351, 447

Pilyugin, L. S., \& Thuan, T. X. 2005, ApJ, 631, 231

Reach, W. T., Boulanger, F., Contursi, A., \& Lequeux, J. 2000, A\&A, 361, 895

Reach, W. T., et al. 2005, PASP, 117, 978

Rieke, G. H., et al. 2004, ApJS, 154, 25

Roberts, M. S., Hogg, D. E., Bregman, J. N., Forman, W. R., \& Jones, C. 1991, ApJS, 75, 751

Roche, P. F., Aitken, D. K., Smith, C. H., \& Ward, M. J. 1991, MNRAS, 248, 606

Rots, A. H. 1980, A\&AS, 41, 189

Roussel, H., et al. 2006, ApJ, 646, 841

Savage, B. D., \& Sembach, K. R. 1996, ARA\&A, 34, 279
Sheth, K., Vogel, S. N., Regan, M. W., Thornley, M. D., \& Teuben, P. J. 2005, ApJ, 632, 217

Smith, J. D. T., et al. 2007, ApJ, 656, 770

Strong, A. W., \& Mattox, J. R. 1996, A\&A, 308, L21

Sturm, E., Lutz, D., Tran, D., Feuchtgruber, H., Genzel, R., Kunze, D., Moorwood, A. F. M., \& Thornley, M. D. 2000, A\&A, 358, 481

Tosi, M., Sabbi, E., Bellazzini, M., Aloisi, A., Greggio, L., Leitherer, C., \& Montegriffo, P. 2001, AJ, 122, 1271

Tuffs, R. J., Popescu, C. C., Völk, H. J., Kylafis, N. D., \& Dopita, M. A. 2004, A\&A, 419, 821

Tüllmann, R., Rosa, M. R., Elwert, T., Bomans, D. J., Ferguson, A. M. N., \& Dettmar, R.-J. 2003, A\&A, 412, 69

Voit, G. M. 1992, MNRAS, 258, 841

Walter, F., et al. 2007, ApJ, 661, 102

Waters, L. B. F. M., et al. 1998, A\&A, 331, L61

Weingartner, J. C., \& Draine, B. T. 2001a, ApJ, 548, 296 (WD01) 2001b, ApJS, 134, 263

Werner, M. W., et al. 2004, ApJS, 154, 1

Whittet, D. C. B., Bode, M. F., Longmore, A. J., Adamson, A. J., McFadzean, A. D., Aitken, D. K., \& Roche, P. F. 1988, MNRAS, 233, 321

Wu, Y., Charmandaris, V., Hao, L., Brandl, B. R., Bernard-Salas, J., Spoon, H. W. W., \& Houck, J. R. 2006, ApJ, 639, 157

Young, J. S., \& Scoville, N. Z. 1991, ARA\&A, 29, 581

Zubko, V. G., Mennella, V., Colangeli, L., \& Bussoletti, E. 1996, MNRAS, 282,1321 